\title{
Dual-functional superwettable nano-structured membrane: from ultra-effective separation of oil- water emulsion to seawater desalination
}

By

Shiyi Chen

A Thesis submitted to the Faculty of Graduate Studies of The University of Manitoba

In partial fulfillment of the requirements of the degree of

MASTER OF SCIENCE

Department of Mechanical Engineering

University of Manitoba

Winnipeg

(C) Copyright 2020 by Shiyi Chen 


\section{Abstract}

Many research works are striving to purify oil contaminated wastewater. Membrane with distinct affinity toward oil or water is one of the most promising method for oil-induced wastewater treatment. However, with the emulsifier present in most wastewater, the immiscible oil/water mixture is more difficult to be separated. Meanwhile, making use of the most abundant resource of seawater, solar vapour generation has emerged as a sustainable method for water distillation. However, traditional solar steam technologies still rely on expensive or cumbersome optical infrastructure or suffer from low energy efficiency. A water purification system with the ability to treat industrial oily wastewater and distill seawater is yet to be developed. In this thesis, a robust electrospun nanofibrous poly(vinyl alcohol) membrane (NPM) decorated with slow oxidization of polypyrrole nanoparticles was synthesized to realise dual-functional water purification. Scanning electron microscopy (SEM) was utilized to characterize the morphology of the NPM. Fourier-transform infrared spectroscopy (FTIR) was used to verify the chemical composition of the NPM. Contact angle tests were carried out to validate superhydrophilicity/underwater superoleophobicity of the NPM. Repeated oil/water emulsion separation tests were conducted to obtain the results of separation efficiency, separation flux and anti-fouling property of the NPM. UV-vis was utilized to test the light absorption ability of the NPM. Simulated sun tester was used to test the solar vapor generation capacity of the NPM. In summary, the NPM is capable of separating sundry organic solvents-water emulsions with a separation efficiency of over $99.99 \%$. The NPM is also capable of desalinating the seawater under one sun solar radiation with an evaporation rate of $2.87 \mathrm{~kg} \mathrm{~m}^{-2} \mathrm{~h}^{-1}$. 


\section{Acknowledgements}

Extensive thanks must be given to Dr. Malcolm Xing of the Department of Mechanical Engineering at University of Manitoba for his much appreciated guidance and academic support throughout my studies, without whom this research work would not be conducted successfully.

I would also like to thank the rest of my thesis committee: Dr. Subramaniam Balakrishnan and Dr. Qingjin Peng for their insightful comments and inspiration.

Thanks also to Dr. Wen Zhong and Dr. Yuqing Liu for their support in many of the practical elements of this work as well as the technical trainings.

My thanks also go to my lab mates for their insights from our discussions and assistance during the experiments.

Last but not least, my thanks go to my friends and family for their unconditional support during my graduate study. 


\section{Table of Contents}

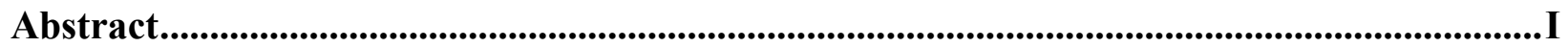

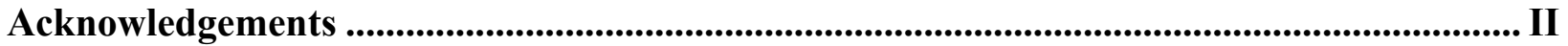

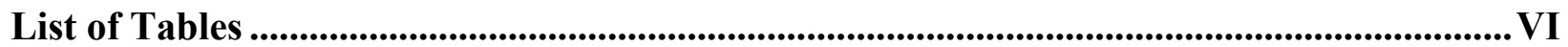

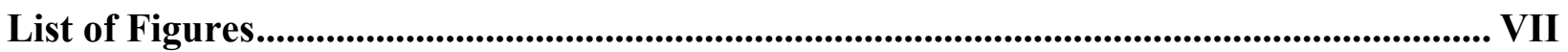

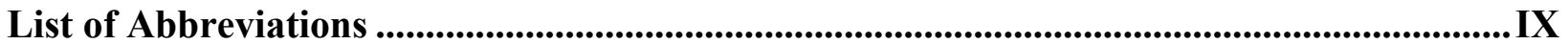

Chapter 1 Introduction ..................................................................................................................... 1

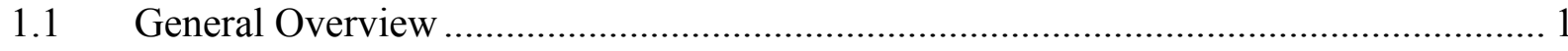

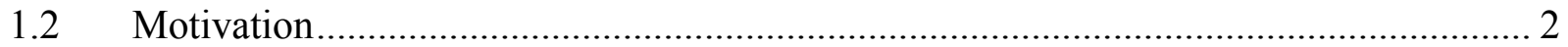

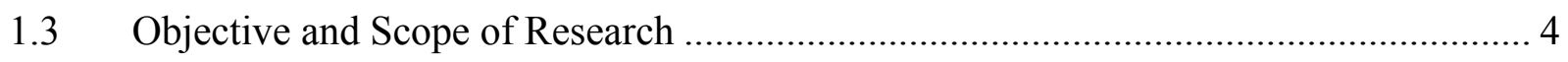

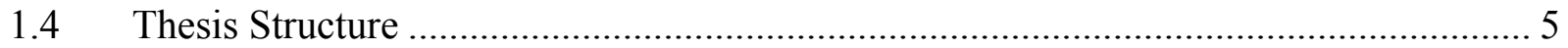

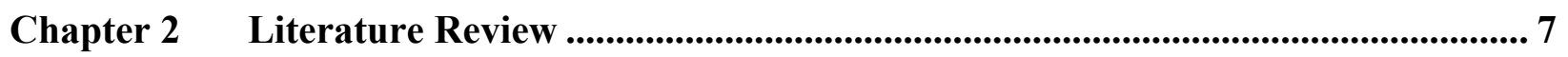

2.1 Superwettable Oil/water Separation Materials ……….............................................. 7

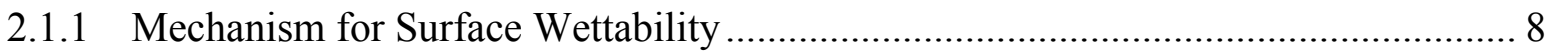

2.1.2 Oil/water Separation Materials in Filtration ........................................................ 10

2.2 The Electrospun Membranes for Oil/water Separation ............................................ 10

2.2.1 Basic Mechanism of Electrospinning ……........................................................ 10

2.2.2 Basic Electrospinning Parameters...................................................................... 11

2.2.3 Wettability in Electrospun Membranes .............................................................. 12

2.2.4 Polymeric Electrospun Membranes ………………….......................................... 12

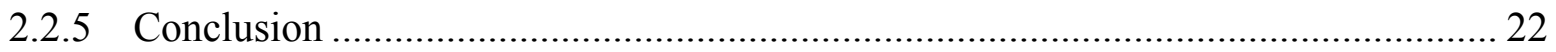

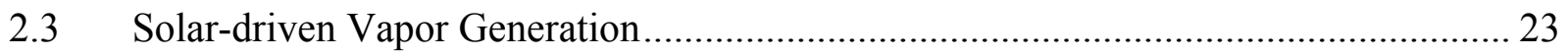


2.3.1 Photo-thermal Materials for SVG ...................................................................... 25

2.3.2 Micro-/nanoscale Structures for Enhanced Light Absorption .................................. 27

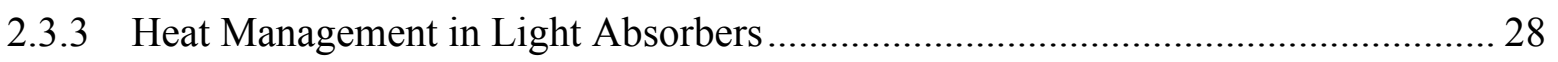

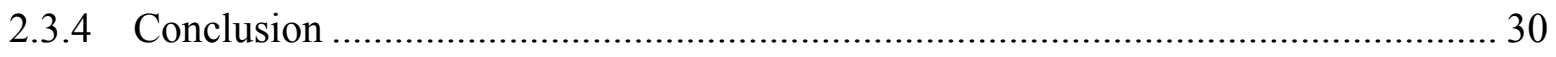

Chapter 3 Dual-functional Superwettable Nanofibrous Membrane................................. 32

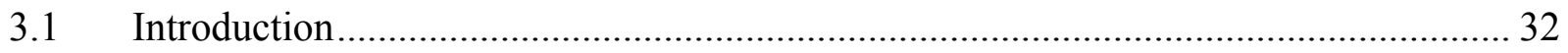

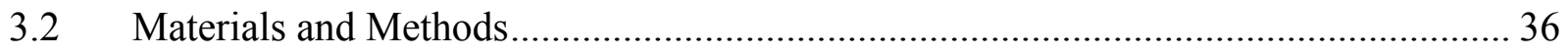

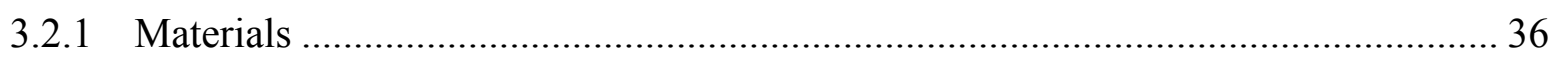

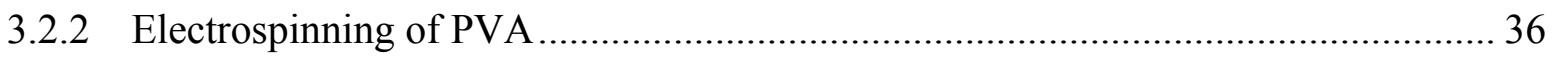

3.2.3 Crosslinking of Electrospun PVA-GO Membrane ………...................................... 36

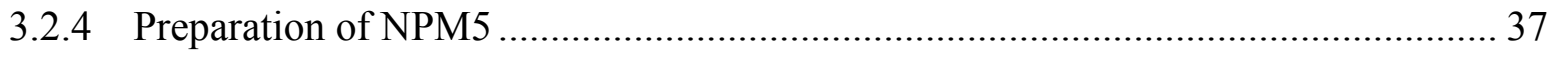

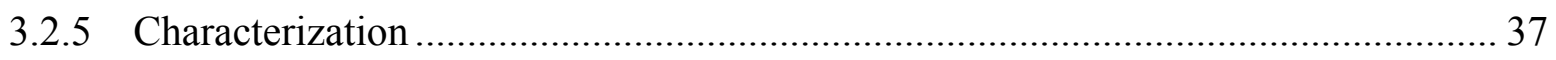

3.2.6 Oil/water Emulsion Preparation and Filtration Evaluation...................................... 38

3.2.7 Solar Vapor Generation Experiment........................................................................ 39

3.2.8 COMSOL Heat Transfer Simulation .................................................................. 40

Chapter 4 Membrane Characterization...................................................................................... 41

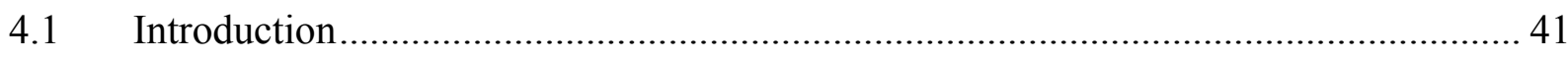

4.2 Hierarchical Nanostructure of the Membrane................................................................ 42

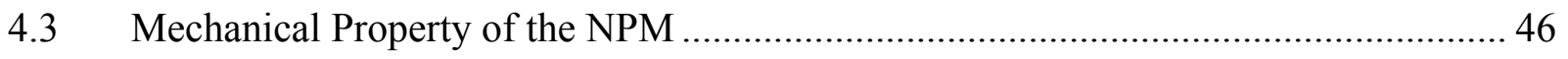

4.4 Water and Underwater Oil Contact Angle Tests ........................................................... 50

4.5 Organic Solvent Contact Angle and Sliding Angle Test ............................................... 52

Chapter $5 \quad$ Oil/water Separation Performance of the NPM.....................................................56

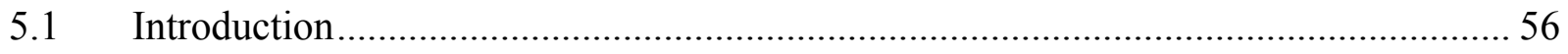


5.2 Separation Efficiency of Sundry Organic Solvent/water Emulsion ......................... 57

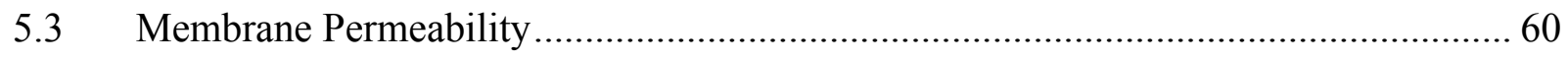

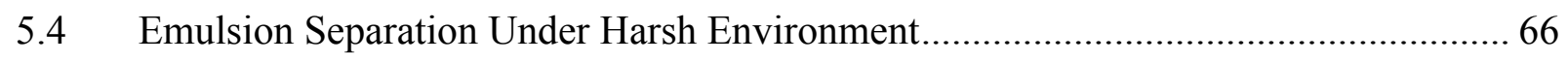

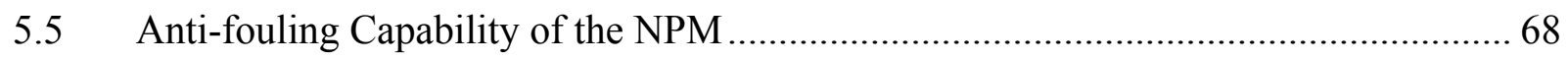

5.6 Super Wettability Mechanism....................................................................... 71

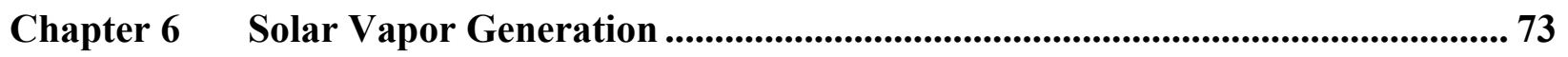

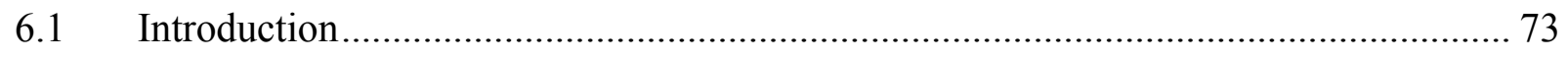

6.2 The Solar Steam Installation Under Various Illumination Concentration.................. 73

6.3 Effect of PPy on Solar Vapor Efficiency .......................................................... 75

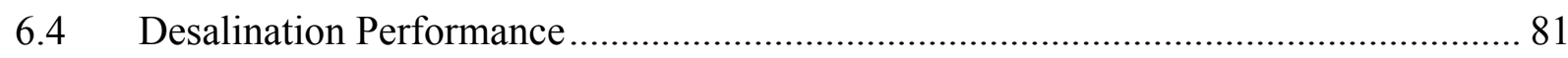

6.5 On-site Solar Vapor Generation Performance ............................................... 83

Chapter 7 Conclusion and Future Works............................................................... 86

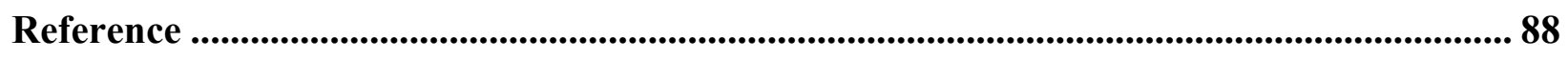

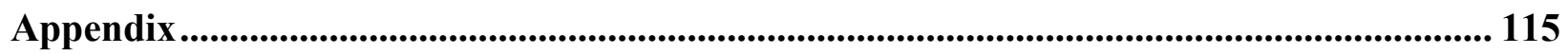




\section{List of Tables}

Table 3-1. The performances of oil water separation and solar steam generation of different

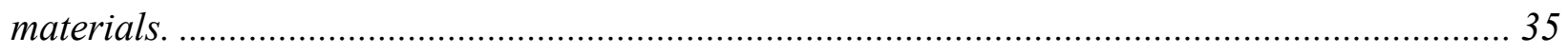

Table 5-1. The composition of artificial seawater (The total mass of the compound and the

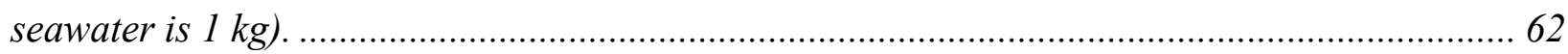




\section{List of Figures}

Figure 4-1. (a) Schematic Illustration of the preparation process of the NPM membrane and the dual functions of the membrane. (b) the on-site set-up illustration for seawater desalination.... 43 Figure 4-2. Photographs of PVA membranes without (up) and with (down) crosslinking of 25\% glutaraldehyde after dissolving in distilled water (DW) for $1 \mathrm{~h}$ 44

Figure 4-3. Surface morphological changes of the NPM, tensile stress versus strain curves of the crosslinked of the NPM, contact angles and FT-IR spectrum of NPMs. 45

Figure 4-4. Tensile stress strain curves of the crosslinked PVA membranes mixed with 0.1\%, $0.2 \%, 0.3 \%$ GO and no $G O$. 47

Figure 4-5. SEM images NPM1 to NPM5. 49

Figure 4-6. Underwater Oil Contact Angles and underwater sliding Angles of the NPM against different oily droplets. 54

Figure 4-7. Underwater Oil Contact Angles of the NPM against different oily droplets after immersing the membrane in artificial seawater for 30 consecutive days 55

Figure 4-8. Dynamic sliding angle test of NPM1 with a droplet of dichloromethane 55

Figure 5-1. Oil/sea water emulsion separation installation with optical microscopy of emulsions before and after separation, together with the separation flux and Particle size distribution of the petroleum ether-in-water mixture. 59

Figure 5-2. The separation flux of a sequence of surfactant-free and surfactant-stabilized oil-inseawater emulsions penetrating through the membrane. 63 
Figure 5-3. Distribution of oil drops with different diameter ranges with and without emulsifier stabilization and images of the surfactant stabilized 10\% oil-in-water emulsions on day 1 and

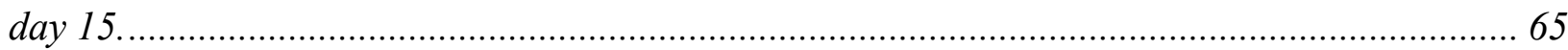
Figure 5-4. Anti-fouling and resilience characteristics of the NPM.................................... 70 Figure 6-1. Schematic set up for solar-vapor desalination unit used in the experiment and the summary of energy efficiencies and evaporation rates of NPM with various degree of PPy coating........ 79

Figure 6-2. UV-vis NIR spectra comparison of NPM, swelling ratios of NPM1, NPM3 and NPM5 and IR images of the thermal dispersion of the NPM5. 79

Figure 6-3. The calculated equivalent enthalpy of bulk water, electrospun PVA membrane and PVA-PPy membrane based on the evaporation rates in dark condition. 80

Figure 6-4. The salinity test result using NPM5 with salinity result before desalination in comparison to after desalination and the evaporation rate and energy efficiencies of NPM5 under various seawater conditions with 1 sun illumination. 82

Figure 6-5. COMSOL model of calculated device with 2D indirect contact and $3 D$ artificial transpiration. 85 


\section{List of Abbreviations}

BSA - Bovine serum albumin

CA - Contact angle

DLS - Dynamic light scattering

DW - Distilled water

FRR - Flux recovery ratio

FTIR - Fourier-transform infrared

GO - Graphene oxide

HA - Humic acid

NPM - Nanofibrous poly(vinyl alcohol) membrane

PAA - Poly(acrylic acid)

PAN - Polyacrylamide

PBZ - Polybenzoxazine

PEO - Poly(ethylene oxide)

PES - Polyethersulfone

PMAA - Poly(methacrylic acid)

PMMA - Poly(methyl methacrylate)

PNIPAM - Poly(N-iso-propylacrylamide)

POSS - Polyhedral oligomeric silsesquioxane

PPy - Polypyrrole 
PS - Poly(styrene)

PVA - Poly(vinyl alcohol)

SA - Sliding angle

SDS - Sodium dodecyl sulfate

ZIF-8 - Zeolite imidazole fram 


\section{Chapter 1 Introduction}

\subsection{General Overview}

With the pressure of climate change, the global water shortage will have repercussions of economic development, food supply and human survival itself. According to a recent report by World Health Organization on water scarcity, 2.2 billion people lack access to safely managed drinking water because of the exploded population, the shrinkage and contamination of freshwater resources[1,2]. The dire warning of freshwater scarcity has gained impetus for many researchers to find new freshwater resources, while harnessing the ever-increasing water contamination problem.

To tackle the issue of water contamination, both academia and industry have developed methods to treat the crude oil contaminated water, including in situ burning[3], chemical dispersants degradation[4] and vacuum suction[5]. However, these methods may suffer from high cost, secondary pollution or low efficiency. Meanwhile, it is highly sought after to remove not only crude oil, but also non-water-soluble organic solvents as the rapid industrialization has led to more wastewater emission. Therefore, it is of great importance to develop a feasible method that can selectively remove the oil or non-soluble organic solvents in an efficient, sustainable manner without bringing secondary pollution.

From the materials perspective, superwettable materials with specific affinities for oil or water have drawn tremendous attention and believed to be a powerful solution to oil spill or oil 
contamination[6-10]. Recently, metallic meshes[11, 12], fabrics[13], sponges[14-16] or polymeric membranes[17, 18] with hydrophilic/oleophobic or hydrophobic/oleophilic properties have been developed to separate oil/water mixture. Most of these advanced materials exploited the synergistic effect of morphology and surface chemistry to tune the wetting behavior towards water or oil[19]. For instance, the surface tension determines a material's affinity towards oil or water. Such affinity can be magnified by manipulating the surface architecture of the material, tuning the wetting behavior from i.e., hydrophilic to superhydrophilic[20, 21].

Apart from treating contaminated water sources, many research works have been devoted to finding new water sources to address the freshwater crisis. Desalination of the abundant resource of seawater is considered a plausible method[21-23]. Using the ubiquitous solar energy, solar vapor desalination has attracted tremendous attention due to its omnipresence and transformative industrial potential[24]. Recently, localized heating at liquid/air interface implemented solar steam generation was proposed as an alternative to traditional bulk heating steam generation[25, 26]. The solar-driven localized heating vapor generation involves higher efficiency of solarthermal conversion and less thermal loss. To date, advancement of this technique lies in several aspects: light absorption, light-thermal conversion, water transportation and evaporation[27, 28]. Designing a steam generation system with thorough consideration of all these factors as well as industrial implementation is challenging but rewarding.

\subsection{Motivation}

A series of "water removing" or "oil removing" materials were synthesized via constructing superhydrophobic/superoleophilic[29-31] or superhydrophilic/superoleophobic[32-35] systems. Both methods have generally shown a prominent separation efficiency of over $99.9 \%[36,37]$. 
However, because of the higher density of water compared to oils, separation process can be quickly baffled as water can form a barrier layer on a superhydrophobic surface. Meanwhile, from industrial wastewater to crude oil spill, the separation targets are usually water-rich oil-inwater mixtures, making superhydrophobic materials unsuitable for the situation. Therefore, superhydrophilic/superoleophobic materials are the more promising candidate in terms of practicality and viability. Many strategies have been proposed to develop surface textures with superoleophobicity[38]. Jiang et al. reported a $\mathrm{Cu}(\mathrm{OH})_{2}$ nanowire-haired copper mesh with high flux oil/water separation property[33]. The superhydrophilicity was achieved by chemically etching the copper mesh to acquire a high-surface-energy nanowire surface. However, the separation efficiency is greatly dependent on the mesh size as the emulsion droplet size should not exceed the pore size, making surfactant stabilized nano-droplet emulsion impossible to separate. Some recent studies have successfully demonstrated surfactant stabilized emulsion separation by creating nano-pores in the membranes[36, 39]. However, the mechanical strength issue of the membrane remains to be tackled as the materials used to endow oil repellence are far from robust, i.e. hydrogels[40], photocatalytic nanoparticles[41]. Additionally, the capability for the material to function under harsh environments are generally not addressed.

Another solution to water scarcity is to increase the freshwater supply. Making use of the most abundant resource of seawater, solar vapour generation has been emerged as a sustainable method for water distillation. However, traditional solar steam technologies still rely on expensive or cumbersome optical infrastructure or suffer from low energy efficiency. Solar desalination of seawater is a promising methodology with least impact on environment $[19,42-$ 45]. Traditional seawater desalination methods such as thermal distillation and reverse osmosis membrane filtration are highly energy-demanding or with low efficiency. Recently, plasmonic 
nanoparticles for solar steam generation has attracted tremendous attention due to its high photothermal conversion efficiency and localized heating[46-48]. The prominent localized heating is due to the subsequent nonradiative relaxation of metals, causing absorbed energy to be converted into heat[49]. However, noble metals used for plasmon-mediated solar desalination, i.e. platinum and gold, are not cost-effective for large scale application[47, 50, 51]. Other more abundant metals generally have higher plasma frequency, resulting in significant plasmonic resonance only for a limited range of solar spectrum[52].

In that regard, it is highly sought-after to develop a facile method that is simultaneously superoleophobic, capable of light-to-heat conversion and low thermo-conductivity that is good for both efficient immiscible industrial wastewater purification and large-scale solar steam generation.

\subsection{Objective and Scope of Research}

Regarding the previously discussed issues, the objective of this thesis is to develop effective water purification materials that are capable of purifying oil contaminated wastewater, desalinization of seawater with solar energy and scavenge water from atmosphere. The objectives of this research work are as follows:

1. To develop a porous nanofibrous membrane with underwater superoelophobicity/ superhydrophilicity

2. The membrane should achieve highly effective separation for surfactant-stabilized immiscible oil/water mixture

3. The membrane should possess robustness and durability under harsh working conditions

4. To achieve broadband light wavelengths absorption under solar radiation 
5. To achieve high light-to-thermal energy conversion rate enabled effective solar steam generation for seawater desalination

The synthesized membrane was characterized by SEM, FTIR to examine the morphology and composition. Tensile tests were carried out to examine the mechanical properties of the membrane. Oil and underwater contact angle tests were used to demonstrate superhydrophilicity and underwater superoleophobicity of the membrane. The separation efficiency, separation flux data was obtained via oil/water separation test. Underwater oil sliding angle test was carried out to examine the oil adhesion property of the membrane. Cyclic separation test was conducted to test the anti-fouling property of the membrane. UV-Vis spectrometer was utilized to test the light absorption potential of the membrane. Simulated sun tester was utilized to measure the evaporation rate and energy efficiency of the membrane.

\subsection{Thesis Structure}

This dissertation is composed of seven chapters. The layout is demonstrated as follow:

- Chapter 1 briefly introduces the background of freshwater crisis and several feasible solutions to address the problem, followed by motivation and objective of this research.

- Chapter 2 contains the literature review of superwettable materials and solar vapor generator serving the purpose of oil/water separation and seawater desalination, respectively.

- Chapter 3 provides detailed experimental procedures of the synthesis of NPM

- Chapter 4 is comprised of the characterization of the NPM. 
- Chapter 5 presents analysis and discussion of the oil/water separation performance of the NPM.

- Chapter 6 presents solar vapor generation performance of the NPM.

- Chapter 7 summarizes the results and conclusion of this research work and suggestions for potential future work. 


\section{Chapter 2 Literature Review}

\subsection{Superwettable Oil/water Separation Materials}

The rapid industrialization and growing energy demand have exacerbated the crude oil exploitation, coming along with reoccurring oil spill accidents as well as more severe wastewater pollution. The crude oil leakage accident or inappropriate oil discharge has serious ramification on vulnerable ecosystems. For example, in 2010 around 600,000 tons of crude oil was leaked into Gulf of Mexico, causing catastrophic damage to marine ecosystem and public health. More recently, the Sanchi oil tanker collision has caused over 136,000 tons of natural-gas condensate leaking into South China Sea. The leakage has immediate toxic effect on species in that habitat, along with lasting ecological consequence[53]. To remediate the after-math of such incidents, it is of urgent importance to develop feasible method to achieve efficient oil/water separation. Traditional oil/water separation methods, i.e. skimming, centrifuge, absorption and in-situ burning, suffer from numerous shortcomings including high cost, low efficiency, and having negative impact on environment. More importantly, how to reclaim the oil separated from water is highly looked-for as crude oil is a precious resource and should be recycled for future usage. Therefore, finding solutions to overcome above mentioned limitations in oil/water separation should be actively pursued. Recently, scientists and engineers have developed a series of methods featuring materials with specific affinities to water or oil. These special wettable materials are showing promising outlook for selective water or oil removal. Briefly, such advanced wetting behavior is achieved through the synergistic effect of surface texture and surface chemistry. Detailed mechanisms regarding superoleophilicity/superhydrophobicity or superoleophobicity/superhydrophilicity will be explained later. 
As one of the pioneering works in the field, Feng et al. proposed a proof-of-concept superoleophilic/superhydrophobic material aiming at oil/water mixture separation. Numerous materials with distinct affinity to water or oil have been proposed successively. Generally, the materials fall into two categories: filtration and absorption materials. Filtration materials, i.e. membranes, textiles and meshes, allow one phase to penetrate through, while blocking the other phase above, therefore achieving selective removal. Absorption materials on the other hand, usually have porous morphology and are capable of absorbing one phase and repel the other while in a mix of oil and water environment. Both categories with their representative works will be thoroughly discussed in the following paragraphs. The wettability, innovation, highlights and limitations will also be reviewed.

\subsubsection{Mechanism for Surface Wettability}

The wettability of a material is normally gauged by contact angle (CA) of a droplet (e.g. water) against the surface of aforementioned material. By definition, hydrophobicity means the CA of a water droplet on the substrate is larger than $90^{\circ}$ while hydrophilicity means the contact angle is smaller than $90^{\circ}$. To make a material superhydrophobic or superhydrophilic, the contact angle needs to be larger than $150^{\circ}$ or smaller than $10^{\circ}$, respectively. To explain why a material is hydrophobic or hydrophilic, Young's equation is commonly used[54]:

$$
\cos \theta=\frac{\gamma_{S V}-\gamma_{S L}}{\gamma_{L V}}
$$

Equation 1

in which $\gamma_{S V}$ is the interfacial energy of the interface of solid and vapor phases, $\gamma_{S L}$ is the interfacial energy of the interface of solid and liquid phases and $\gamma_{L V}$ is the interfacial energy of liquid-vapor phase.

Normally, the surfaces of the materials are not absolutely smooth and has some degree of roughness. The surface architecture has a large impact on the wetting behaviour of the materials, 
besides the surface chemistry. There are three wetting behaviours of a material with rough surface: Cassie state, transition state and Wenzel state[55-60]. In a Wenzel state, the liquid phase will wet the porous surface and the grooves of the rough microstructure will be filled by liquid. Wenzel stated that the wettability of the materials is amplified by the porous nature of the substrate because of the increased surface area of a rough surface. Consequently, the Young's equation is modified[61]:

$$
\cos \theta^{*}=\frac{R\left(\gamma_{S V}-\gamma_{S L}\right)}{\gamma_{L V}}=r \cos \theta
$$

in which $\theta^{*}$ represents the apparent $\mathrm{CA}$ of a droplet on a textured substrate, $\theta$ represents the CA on a smooth subsrate, which is also the CA in Young's equation. R represents the roughness factor, which is the fraction of real surface area to projected area.

When the grooves inside of the rough surface repel the liquid phase so that the droplet does not wet the grooves, the droplet will sit on the top of the rough surface, with air trapped in the grooves. In this case, Cassie et al. carried out another equation to describe such wetting state[60]:

$$
\cos \theta^{*}=f \cos \theta+f-1
$$

in which $\theta^{*}$ and $\theta$ still represent the apparent $\mathrm{CA}$ of the droplet on the rough substrate and intrinsic CA on the smooth substrate, respectively. The area fraction of the surface is referred as the $f$.

There is another wetting state when the droplet partially fills the grooves of a textured surface, which is considered a transition state of Cassie and Wenzel states[57, 62-65]. Wenzel state usually exhibits extremely high adhesion between substrate and the liquid droplet, while on the contrary, Cassie state usually shows extremely low adhesion. As for transition state, the adhesion can be low or high, depending how much of the liquid fills the grooves and how much sit on the surfaces of the substrate[66-68]. 
In Cassie state, the contact angle is reduced because of the air trapped in the rough microstructure. The air phase in Cassie models can be replaced by water phase. For example, when a hydrophilic surface is immersed in water, the porous textured surface is entirely wetted. when oil droplets are put on a substrate, instead of the air trapped in the grooves, water phase will replace the vapor phase and act as a cushion between the oil phase and the substrate. In this state, oil droplets will be entirely repelled from the material and sit on the top of the microstructure. The underwater Cassie model is originally used to explain the oil-repellent characteristic of fish scales[17, 34, 69-71], which inspired invention of various superoleophobic materials.

\subsubsection{Oil/water Separation Materials in Filtration}

Among all of the oil/water separation materials for filtration, polymer membranes are one of the most used type because of their versatility and scalability. The major drawback of membrane is the limited mechanical strength. In addition, membranes usually have small pores, which requires raised pressure to maintain a reasonable flux.

\subsection{The Electrospun Membranes for Oil/water Separation}

\subsubsection{Basic Mechanism of Electrospinning}

Electrospinning is an electrodynamic technique that has long been used for fabricating nonwoven nanofibrous membrane. During electrospinning, the polymeric droplets are pushed from a needle and electrified to generate a jet[72]. The jet is further stretched and solidified to form nanofibers, which deposit on the collector screen to form a membrane[73]. Typically, an electrospinning system is comprised of three elements: a capillary tube (typically a syringe to store precursor solution and a spinneret), a direct current power device with high voltage and a collector. At the beginning of electrospinning, the polymer droplet at the tip of the spinneret 
transform to a conical shape due the applied electric field[74]. The electrostatic repulsive force at the tip of Taylor cone overcomes surface tension of the droplet, once electric field passes a threshold value.[75] Afterwards, the jet transforms from a straight line to multiple jets due to the rapid whipping. In the end, continuous nanofibers randomly deposit on the collector to generate a non-woven membrane.[76]

\subsubsection{Basic Electrospinning Parameters}

Polymer properties, solution properties and operation parameters all play critical roles in the architecture of the nanofibers, including diameter of the nanofibers, thickness and porosity of the membrane. Polymer properties entail solubility, molecular weight and molecular weight distribution. Operation factors including flow rate, ambient factor, electric field intensity also have an impact on the electrospun nanofibers. Surface tension, viscosity and conductivity of spinning liquid are parameters to be considered for optimal membrane synthesis. Take diameter of the nanofibers as an example, it can be significantly reduced if the viscosity of the polymer is decreased. Nevertheless, when the viscosity reaches a lower threshold, there will be beads on the nanofibers, which endows the membrane with higher porosity. In addition, the high aspect ratio of nanofibers endows the membrane with high flexibility and robustness.[77] Meanwhile, tuning the conductivity of the solution can result in finely fabricated nanofibers with a diameter of 20 $\mathrm{nm}$, which largely reduces the porosity of the electrospun membrane.[78-80]

Recently, several novel designs are developed in the electrospinning device to construct various configurations and structures of nanofibers. The majority of the designs are based on advancing the collector and the spinneret to fabricate Janus, core-shell, pattern, anisotropic, hollow and 3D structures.[81] These new nanostructures notably expanded the versatility of the membranes for oil/water separation.[82] 


\subsubsection{Wettability in Electrospun Membranes}

Wettability is the wetting behavior of liquid on the solid substrate. When it comes to oil/water separation technique, wettability of a material is the most critical factor to assure efficiency of the separation.[83] Most research works utilize the synergistic effect of surface chemistry and surface architecture to manipulate the wettability of materials.[84] Surface chemistry is the intrinsic characteristic that controls the free energy of the surface. Surface architecture also determines the wettability of the membrane, which can be explained by Cassie or Wenzel model in the previous chapter.[61, 85]

Up to now, there have been more than 200 types of synthetic or natural polymeric electrospun nanofibers.[86-88] Inorganic materials (e.g. ceramic, metal and carbon) can also be electrospun into nanofibers, indicating the versatility and potentials to tune the surface tension of the membrane. Moreover, micro-roughness and nano-roughness can be inherently constructed in electrospun membrane. $[89,90]$ These advantages enable the elestrospun membrane with great capacity in adjusting wettability.

Generally, nanofibrous membranes with selective wettability are used to separate oil/water emulsion, which means the membranes are affiliative to one phase (oil or water) and show repellence to another phase. For example, when using a hydrophobic-oleophilic membrane for micelle emulsion separation, oil can permeate the rough nanostructure with no air reversed as the material has a strong affinity towards oil. As a result, water can be divided from oil at the solid interface.

\subsubsection{Polymeric Electrospun Membranes}

Polymers possess many advantages including great processability, high flexibility, low cost, which make them ideal candidates for oil/water separation.[82] To date, numerous polymeric 
membranes aimed for successful oil-water mixture or emulsion/micelle separation have been synthesized. The polymer membranes can be categorized in mono-layer and multi-layer ones based on the number of layers in the membrane. It is worth noting that inorganic nanoparticles can be incorporated into the polymer-based membrane, hence the polymeric membrane are not exclusive to membranes only composed of polymers and they will be discussed in the following section.

Mono-layer structured membrane is the most straightforward and common type applied in oil/water separation. Generally, selective wetting behavior in mono-layer structured membranes is achieved by tuning surface morphology and surface chemistry. Till now, there are three kinds of wettability in mono-layer polymeric membranes: hydrophobicity/oleophilicity for oilremoving membrane, hydrophilicity/oleophobicity for water-removing membrane, and switchable wettability for controllable water/oil removing.

\subsubsection{Membranes with Hydrophilicity/underwater Oleophobicity}

Theoretically, hydrophilicity/oleophobicity in mono-layer membrane is utilized to selectively removing water from the emulsion. Nonetheless, because of the high surface energy of water compared to oil $\left(\gamma_{\text {water }}=72.8 \mathrm{mN} \mathrm{m}^{-1}\right.$ and $\gamma_{o i l}$ is usually $\left.<36 \mathrm{mN} \mathrm{m}^{-1}\right)$, [56, 91] hydrophilicity and oleophobicity is difficult to obtain. Several works have been inspired by nature (e.g. fish scale) and utilizing polymers with high surface tensions to achieve hydrophilicity and underwater oleophobicity.[69, 92] Generally, underwater oleophobic/hydrophilic nanofibrous membranes are fabricated via direct electrospinning, which is a facile method for single-texture membrane. When the micro/nano-texture or hierarchy of the membrane is more sophisticated, biomimetic methods including surface self-assembly, colloid electrospin and wet chemical reaction are utilized. 
The polymers with high surface energy are high in polar groups (e.g. carboxyl group, hydrogen bond, amidogen) and are prone to swelling, dissolving and water absorbing. Considering that the working condition of the membranes is underwater, as-electrospun membranes need to be processed before application. Till now, nanofibrous membrane with hydrophilicity and oleophobicity are developed via three methods. The first and most utilized method is directly using intrinsically hydrophilic polymers as spinning material. Polyacrylonitrile as a synthesic polymer with excellent ability for electrospinning and accessibility is one of the most promising material. The as-electrospun polyacrylonitrile membrane can be treated with sodium hydroxide to further control the wettability.[93] After the treatment of sodium hydroxide, the nitrile groups in polyacrylonitrile transform to carbonyl group and hydroxyl during the hydrolysis process, thus enabling the membrane with hydrophilic/underwater oleophobic property. Due to the partial hydrolysis, cyan groups are still present in the polymer, which prevent the membrane from dissolving and swelling. More recently, natural polymers with intrinsic hydrophilicity, low cost and insolubility have attracted significant attention as a candidate for selectively removing water in oil/water mixture.[87, 94, 95] For instance, Lim et al. synthesized a cellulose membrane through direct electrospinning.[96] Owing to the chemical resistant property and hydrophilic nature of cellulose, the membrane exhibited great resilience under harsh environment while maintaining high efficiency in separating oil from water. Other research work utilized silk fibroin as a material for electrospinning.[97] Given the hydroxyl group and amine group in silk and the nanostructure of electrospun membrane, the membrane displayed an superhydrophilicity (exhibiting water $\mathrm{CA}$ of $0^{\circ}$ ) as well as underwater superoleophobicity (exhibiting underwater oil CA beyond $150^{\circ}$ ). These prominent properties are vital in immiscible oil/water micelle 
separation or other non-soluble organic solvent/water emulsion separation (e.g. toluene, chloroform, dichloromethane, etc.).

The second method that can effectively reduce solubility/swelling of as-electrospun membrane is crosslinking treatment, which results in lower percentage of hydrophilic function groups. Poly(vinyl alcohol) (PVA),[98, 99] poly(N-iso-propylacrylamide) (PNIPAM)[100], poly (acrylic acid) (PAA)[101] and poly(methacrylic acid) (PMAA)[102] are polymers that can be electrospun and further crosslinked and still remain hydrophilicity. For instance, a PMAA/cellulose acetate core-shell nanofibrous membrane was reported with amphiphilicity, which can be used to remove surfactant-stabilized micelle emulsions.[102] The core-shell structure was constructed with synchronous phase separation, leading to acetate forming a supporting core while PMAA forming a shell. Afterwards the uniaxial electrospinning, the membrane was thermally treated to create ester bonding between the hydroxyl group in acetate and carboxyl group in PMAA. At the same time, the insolubility of the nanofibers is increased due to the anhydride process in PMAA. At a result, the membrane possesses high efficiency in removing water from immiscible oil/water emulsions.

Most recently, another method to decrease solubility in hydrophilic nanofibrous membrane is blending hydrophobic during electrospinning. Chen and coworkers reported polyethersulfone (PES) solution blending with crosslinked PAA as the spinning solution to fabricate hydrophilic membrane that can be kept intact underwater.[103] The hydrophobicity of PES helped to prevent the dissolving of the membrane while the carboxyl group in PAA maintained the hydrophilicity of the whole structure.

To sum up, direct electrospinning is considered as a facile and efficient method for constructing hydrophilic nanofibrous structures. However, the drawbacks of this method include severe 
fouling issue, poor separation performance due to the lack of hierarchical nanostructure on the nanofibers. Therefore, further approaches are required to achieve nanoscale structure on the surface of nanofibers.

2) Colloid electrospinning. Colloidal electrospinning is a technique that involves spinning solution with nanoparticles of high surface tension and is optimal to fabricate non-woven membranes with superhydrophilicity in one step. The structure and surface tension are able to be finely adjusted by varying the fraction of nanoparticles mixed in the polymer solution. A range of shapes of nanoparticles (e.g. 0D, 1D and 2D, etc.) are electrospinnable. Typical 0D nanoparticles are mainly ceramics, including silicon dioxide, manganese dioxide, and are comprehensively used in constructing nano-protrusion biomimetic structure on the surface of fibers. For example, by mixing silicon dioxide in the polyacrylamide spinning solution, a structure mimicking lotus leaf is constructed with a rough surface, which enables the surface with superhydrophilicity in air. [104] Once the membrane is immersed in oil and water mixture, it is instantly wetted with water and form an aqueous barrier against the oil phase. As a result, the oil droplet is capable of rolling on the membrane surface without permeating through, while water can continuously pass through the membrane. In addition, another type of 0D nanoparticles, metal-organic frameworks has also drawn attention in oil/water separation field because of the substantially high specific surface area and porosity.[105] For example, Lu and coworkers have reported a zeolite imidazole framework (ZIF-8) nanoparticles incorporated in a polyacrylamide nanofibrous system.[106] Decorating ZIF-8 nanoparticles on the nanofibers constructed a hierarchical rough structure with high surface tension that enables the membrane with superoleophobicity under water. Compared to electrospun polyacrylamide (PAN) 
membrane, the ZIF-8/PAN membrane is capable of demulsifying oil/water micelle with a permeating flux of over $900 \mathrm{~L} \mathrm{~m}^{-2} \mathrm{~h}^{-1}$ and a separation efficiency exceeding $99.9 \%$.

Colloid electrospinning is considered as the most facile method to produce hierarchically structured membranes. The drawback of this method is the poor distribution of nanoparticles in the highly viscous spinning solution, which will possibly lead to aggregation of nanoparticles in the membrane. Future development of colloid electrospinning should be devoted to increasing homogeneous dispersion as well as the migration of the nanoparticles in the spinning solution and on fiber surfaces, respectively.

3) Wet chemical reaction. During wet chemical reaction, nanoparticles are synthesized via chemical processes and gradually deposited onto the surface of nanofibers. The fibres act as the skeleton of the synthesized nanoparticles and are soaked in the precursor solution where the reactions take place. Till now, there are three main types of chemical reactions utilized for in situ nanoparticle synthesis: in situ polymerization, hydrothermal treatment and successive ionic layer absorption and reaction.[107-111] Wet chemical reaction is another common method to fabricate multi-structured biomimetic membranes in the laboratory setting. The main obstacle preventing this method from large-scale industrial application is the scalability of the fabricated membrane. In addition, high pressure and temperature are usually required for the generation of nanocrystals during the reaction, resulting in limited choice of substrates and reactants. Moreover, chemical reaction process often requires long reaction time, which is another drawback that hindered its industrial application.

\subsubsection{Membranes with Hydrophobicity/oleophilicity}

Contrary to membrane with hydrophilicity/underwater oleophobicity, hydrophobic membranes have the affinity towards water and can remove oil from the emulsion. The underlying chemical 
principle of these membrane is having surface energy between water $\left(\gamma_{\text {water }}=72.8 \mathrm{mN} \mathrm{m}^{-1}\right)$ and oil $\left(\gamma_{o i l}<35.0 \mathrm{mN} \mathrm{m}^{-1}\right)$.[112] One of the most straightforward method is to directly electrospin polymer with suitable surface energy. Typically, these polymers contain chemical groups with low surface energy, such as $-\mathrm{CH}_{2},-\mathrm{CH}_{3},-\mathrm{CF}_{2},-\mathrm{CF}_{2} \mathrm{H}$ and $-\mathrm{CF}_{3}$.[112] Another method to fabricate membrane with hydrophobicity is to create nanoscale rough surface on the prepared nanofibers, whether using pre-roughing/post-hydrophobization or prehydrophobization/post-roughening method.

1) Direct electrospinning. Direct electrospinning is a facile method utilizing low-surface-energy polymers, either polymers containing or not containing fluorine groups.

The most used polymer without fluorine groups in this method is polystyrene (PS) because of its low cost, accessibility and considerable low surface energy. Lee and coworkers have effectively synthesized a PS electrospun membrane with flexibility.[113] The membrane possesses decent hydrophobicity because of the synergistic effect of the mesh structure and low surface tension and thus is capable of separate micelle solutions. The flexibility of the PS nanofibrous membrane was achieved by copolymerizing more elastic monomer in the styrene molecular chains. As a result, the copolymer possesses the robustness and stiffness of both polymers and the oil/water separation efficiency can reach more than $97 \%$. Other than PS, polyvinyl butyral also possess intrinsic hydrophobic property and can be electrospun onto metallic meshes with different pore size to achieve emulsion separation.[114] The permeation rate can be enhanced by tuning the pore size of the mesh and the permeability of the optimal rate is 10 folds higher than commercial membranes. Aside from PS, other hydrophobic polymers including polysulfone,[115] poly(methyl methacrylate)[116], polyurethane[117] and poly(vinyl chloride)[118] are also 
applied in electrospun membranes aiming for oil/water separation with promising water CA over $120^{\circ}$.

Lately, there is an emergent trend in using highly environmentally friendly and biodegradable materials such as poly(lactic-co-glycolic acid),[119] poly(L-lactide)[120] and poly(ecaprolactone)[121] to fabricate hydrophobic electrospun membranes. You et al., reported a poly(L-lactide) membrane with nanopores on the nanofibers by mixing poly(ethylene oxide) (PEO) in the spinning solution and etching PEO off after direct electrospinning.[122] The resultant nanofibers are left with intersected channels that contribute to $20 \%$ higher oil permeability during the separation process.

As for the polymers containing fluorine groups, poly(vinylidene fluoride) and triptycene-based polyimide are commonly used in fabricating electrospun membrane with hydrophobicity and oleophilicity.[123] For example, Qiu and colleagues reported a poly(vinylidene fluoridehexafluoropropylene) membrane with excellent oil absorption ability owing to the inherent oleophilicity of the polymer and the rough surface of the membrane.[124] As a result, a high water $\mathrm{CA}$ of $134^{\circ}$ and an ultra-low oil CA of $0^{\circ}$ are achieved which enable the membrane for highly efficient emulsion separation. It is also worth mentioning that polymers containing fluorine groups suffer from the drawback of toxicity and may pose harm to the environment and should be handled with cautious in industrial application.

2) Pre-hydrophibization/post-roughening approach. This approach for fabricating hydrophobic/oleophilic membrane consists of two steps: first synthesizing hydrophobic nanoparticles and second electrospinning the fabricated nanoparticles with another hydrophobic polymer solution to achieve rough surface texture of the membrane. Consequently, the membrane shows an exceptionally high water $\mathrm{CA}\left(>150^{\circ}\right)$ and thus demonstrates 
superhydrophobic characteristic. The most used hydrophobic nanoparticles are silicon dioxide and titanium dioxide coupled with organosilation treatment to further decrease the surface energy of the nanoparticles.[125] More novel methods have emerged by using polyhedral oligomeric silsesquioxane (POSS) to construct hydrophobic rough morphology on membranes. As one the works using this method, Cao et al. used POSS and a polymer with intrinsic microporosity to construct a protrusion structure on the nanofibers via colloid electrospinning.[126] The nanofibrous membrane has a water $\mathrm{CA}$ of $155^{\circ}$, which qualifies as superhydrophobicity. This property allows the membrane to separate water/oil micelle with a separation efficiency of $99.95 \%$.

3) Pre-roughening/post-hydrophobization approach. By utilizing this approach, fibers are roughened first and chemically treated afterwards to achieve low surface tension. There are three common roughening methods: in situ crosslinking, self-assembly and wet chemical reaction. As for the chemical modification, the materials are usually small organic molecular containing fluorine groups or methyl groups. More recently, a new polymerized trifunctional phenolic system with low surface energy and high stability, polybenzoxazine (PBZ) was developed as a glue holding the nanofibers and the hydrophobic nanoparticles together.[127] For example, poly(m-phenylene isophthalamide) nanofibers can be immersed in PBZ solution to form Mannich structure during the in situ crosslinking, followed by silicon dioxide nanoparticles attachment to achieve low surface tension.[128] As a result, the resultant membrane possesses superhydrophobicity/superoleophilicity with a water CA of $160^{\circ}$ and an ultralow oil CA of approximately $0^{\circ}$. In addition, the membrane shows low adhesion to water droplets with a sliding angle lower than $5^{\circ}$, which is a crucial characteristic for continuous oil/water separation application. 


\subsubsection{Membrane with Switchable Wettability}

More recently, amphiphilic membranes have attracted much attention due to their switchable wetting behavior.[129] The underlying principle to achieve such property is often associated with external stimuli including changing $\mathrm{pH},[130,131]$ gas,[132] temperature,[133] etc. Several stimuli responding electrospun membranes will be introduced in the following section.

$\mathrm{pH}$ responsive polymers have been widely investigated for the use of wettability-switchable membranes. Poly(2-vinyl pyridine),[134] poly(4-vinyl pyridine)[135] and poly(acrylic acid)[130] have been utilized in this field due to their variations of charges and conformations upon $\mathrm{pH}$ changes. For example, poly(4-vinyl pyridine)-co-poly(methyl methacrylate) electrospun membrane was prepared and showed promising wetting transition property.[135] Once the membrane was immersed in solution with a $\mathrm{pH}$ of 3, the poly(4-vinyl pyridine) was protonated due to the mild base nature of the polymer. The pyridine groups in the polymer chain are extended to the nanofiber surface because of the electrostatic repulsive effect, leading to water being trapped on the nanofiber surface. Once the membrane was immersed in solution with a neutral $\mathrm{pH}$, the protonated polymer chains were conformed, leading to membrane transforming back to the hydrophobic state. The membrane can sustain 5 cycles of repeated wettability switching upon the immersion in acidic and neutral solutions. This property enables the membrane to selectively remove water from oil under a $\mathrm{pH}$ of 7 and remove oil from water once the membrane was treated under a $\mathrm{pH}$ of 3.

As for wettability-switchable polymers with thermo-responsive property, PNIPAM is one of the most applied material due to its convenience and low cost. The heat intrigued free energy change is due to variations of entropy and enthalpy within molecular chains.[133] The major drawback of PNIPAM is its water-soluble nature which hinders its application in oil/water separation. To 
reduce the solubility of PNIPAM, PNIPAM can be copolymerized with an insoluble monomer. PMMA as a non-soluble polymer was copolymerized with PNIPAM and as a result, PMMA in the glassy state can act as the intramolecular crosslinking point thus preventing the PNIPAM from dissolving in water.[136]

Another external-stimuli strategy is gas composition, featuring advantages including renewability, eco-friendliness and low cost. $\mathrm{CO}_{2}$ is commonly used gas in this method and there are several $\mathrm{CO}_{2}$ responsive polymers including poly(N,N-dimethylaminoethyl methacrylate), poly(N,N-diethylaminoethyl methacrylate).[132, 137, 138] The amine groups in these polymers reacted with $\mathrm{CO} 2$ and as a result the polymer chains show a hydrophilic stretched state which allow the water to permeate through. Once the gas is removed from the aqueous environment, the polymer chains switch back to the aggregated hydrophobic state, allowing the oil to pass through. Take Yuan and colleagues' work as an example, poly(N,N-diethylaminoethyl methacrylate) was copolymerized with PMMA, followed by electrospinning to fabricate nanofibrous membrane.[132] The membrane can switch back and forth between hydrophilic/oleophobic state to hydrophobic/oleophilic state with cyclic presence of $\mathrm{CO}_{2}$ and $\mathrm{N}_{2}$ gas, which further realized tunable separation of oil or water from the micelle solution.

\subsubsection{Conclusion}

Until now, electrospinning technique has been matured and considered as a reliable method to fabricate non-woven membrane for filtration and oil/water separation. The flexibility, tuneable wettability and the nanoporous structure make electrospun membranes promising candidate for water treatment. Although there has been notable progress in the advancement of electrospun nanofibrous membrane, several major drawbacks are yet to be solved for more practical applications. 
1) One of the major issue of nanofibrous membrane is the durability. The surface structure is prone to abrasion and damage upon loading, which decrease the separation ability of the oil/water mixture. Additionally, nanoparticles in the membranes can be leached over time and cause secondary contamination during the filtration. Thus, developing robust, stable membranes with nanohierarchical structure is highly demanded.

2) The oil/water emulsion utilized for laboratorial separation testing are simplified, whereas in reality, the contaminated oily water has more complex composition and may contain microorganisms, heavy metals, organic dyes, etc. Thus, membranes developed in laboratory may not meet the separation requirement in industrial settings. Actual environmental condition should be considered in future development of electrospun membranes.

3) One of the major issues of electrospinning is the large-scale application in industry. The high voltage requirement and limited scalability hinder its realistic application in industry. At this point, electrospinning technique is matured in academic setting, but more consideration and adjustment should be taken to achieve more scalable, simple and cost-efficient manufacturing process.

Despite the above challenges in the current oil/water separation technique, there are many opportunities in this field as well. Currently, researches are striving to develop membranes with high anti-fouling performance under continuous operation to treat more viscous heavy oils. A growing number of anti-fouling membranes will be developed with exceptional efficiency and separation performance to advance the field of oil/water separation.

\subsection{Solar-driven Vapor Generation}

Evaporation of water is a ubiquitous phenomenon that is observable from biological metabolism to climate change. In modern industrial settings, evaporation is universally present in heat 
dissipation,[139] power generation[24] and energy storage that involves phase-change.[22] Recently, the field of desalination technique involving water evaporation has attracted much attention to remediate the freshwater crisis.[140, 141] The freshwater produced from evaporation is highly pure but the vaporization process is often powered by fossil fuel, which brings secondary pollution to the environment.[142] Aside from the development of environmentallyfriendly thermal-based distillation technique, one research field is emerged on novel materials with tunable properties for solar-vapor generation and water purification.[143] Making use of the abundant omnipresent resource, solar vapor generation (SVG) purify water with the similar mechanism of natural hydrological cycle in nature.[144] SVG holds the advantages of low cost, environmentally friendliness and fossil fuel-free, which are beneficial properties for water purification.

During the process of vaporization, water transform from the form of liquid to gas at the waterair interface. Generally, two routes can start evaporation: increasing the temperature and decreasing the air pressure. Under ambient environment, water will naturally vaporize because of the lower pressure in air comparing to saturated vapor pressure. Tuning temperature is a straightforward method to regulate the water evaporation behavior. When the temperature increases to a certain point, water molecules escape the interface of the liquid and air. If the temperature exceeds the boiling point threshold, steam will be generated because of the vaporization within bulk water. The process of boiling vaporization is fast but requires massive energy.[145]

Many novel materials for SVG were developed over the last decade that can enhance evaporation at the interface of water and air.[27, 146-150] Most of these materials thrive at conversing photo energy to thermal energy. One of the strategies are adopting solar absorbers with low 
transmittance such as carbon nanotubes and graphene.[51] Common characteristics of these materials are the black appearance and broadband light wavelengths absorption. More recently, much effort has been devoted to enhancing the conversion of light-to-heat efficiency via nanoscale structure and energy-band engineering. Traditional materials for SVG usually suffer from light reflection and heat diffusion, which decrease the light-to-heat conversion efficiency. Therefore, many research works are devoted to increasing the efficiency of heat harvesting. However, SVG requires a high energy input of at least $40 \mathrm{~kJ} \mathrm{~mol}^{-1}$, which severely hinders industrial applications of water vaporization technique. Innovative materials that can enhance the solar-energy harvesting and decrease the energy input of SVG by tuning hydration behaviors are plausible candidates for highly efficient solar-energy enabled water treatment.[151]

\subsubsection{Photo-thermal Materials for SVG}

In order to convert solar energy to thermal energy, the material should be able to absorb incident light and avoid emittance or reflectance of light. Recent years have witnessed the innovation of several materials with photo-thermal conversion property as solar energy absorber such as plasmonic materials, $[152,153]$ semiconductor particles, $[154,155]$ carbonaceous $[51,156]$ and polymers.[157, 158] These materials possess advantages including broadband light spectrum absorption, chemical stability and eco-friendliness.

Polymers consisting $\pi$-conjugated backbones of $\mathrm{sp}^{2}$-bybridized carbon possess split energy levels, giving them large potentials to absorb light.[159] Nevertheless, some conjugated polymers may have unique energy-band configurations, limiting the bandwidth of the light that they can absorb, which affect their light-to-heat conversion efficiency when exposed to natural sun light.[160] Many strategies were developed to broaden the light bandwidth absorption, such as ionic doping, $[161,162]$ co-polymerization[163, 164] and oxidative doping.[165, 166] These strategies 
are designated to increase the near-infrared light absorption by molecular reconstruction, namely, doping with polymers of low fluorescence content. Polypyrrole (PPy) is a commonly used polymer used to increase the light absorption due to the bipolar metallic state of doped pyrrole. The slow oxidation process of pyrrole involves metal cations, such as Fe(III).[167] Moreover, by doping functional groups into PPy molecular chains, nanostructure can be controlled to construct $\pi-\pi$ stacking.[168] Applicable conjugated polymers that have been doped with PPy include poly(3,4-ethylenedioxythiophene):poly(4-styrenesulfonate),[169] dopamine[170] and polyaniline[171] and these doped polymers can absorb broadband light, which is promising for SVG application.

Semiconductors, such as $\mathrm{TiO}_{2}$ and $\mathrm{Ti}_{2} \mathrm{O}_{3}$, are considered as more viable photothermal materials because of their controllable energy band and intrinsic light-to-thermal conversion property. The mechanism of intrinsic thermalizaiton is the formation of excitons when the energy of photons exceeds the band gap, namely, electrons and holes.[172, 173] The electron-hole pairs later reduce to the edge of the conduction and recombine, and the process lead to conversion of irradiative energy to heat. Nevertheless, the recombination of excitons will cause energy loss because of emission, which makes the energy conversion efficiency of semiconducting materials highly dependent on the width of the band gap.[174] Approximately 50\% of electromagnetic radiation energy of sun light is from infrared light.[175] Therefore, increasing the light absorption of the semiconductors around the infrared spectrum will substantially increase the light-to-heat conversion efficiency and recent years have seen many semiconducting light absorbers with heteroatom doping to decrease the energy gap.[176]

$\mathrm{TiO}_{2}$ is a commonly used photo-responsive material for light harvesting because of the compatible energy level configuration with the sum spectrum.[177] The band gap of $\mathrm{TiO}_{2}$ is 
approximately $3 \mathrm{eV}$, which is most responsive to ultraviolet light. Higher efficiency of light-toheat conversion can be achieved by shortening the band gap.[178] Therefore, $\mathrm{Ti}_{2} \mathrm{O}_{3}$ was synthesized with extremely short band gap (approximately $0.09 \mathrm{eV}$ ) which allows it to absorb a broadband wavelengths of sun light from $500 \mathrm{~nm}$ to $2500 \mathrm{~nm}$.[179] Light-absorbing semiconductors with narrow band gap are promising candidates for solar water vaporization considering their excellent light absorption ability under one sun radiation.

\subsubsection{Micro-/nanoscale Structures for Enhanced Light Absorption}

Carbon-rich materials including graphene, carbon nanotubes and amorphous carbon are capable of broadband sun light absorption because of the hybrid bonds with continuous energy level.[180] A drawback of these materials is their high reflectance due to the low emissivity.[181] Thus, many nanoscale or microscale structures have been constructed to reduce the energy loss from reflectance, e.g. porous,[182, 183] aligned[184, 185] and hierarchical structures.[186] One of the strategies to increase the light absorption ability is by tuning the optical path length with different structures.[187] This method reduces the refraction and scattering in the structure even if the intrinsic reflective factor of the material is not changed. In a nanoscale structure, light can be "trapped" in the structure due to the matching of the light wavelength and the dimension of the nanostructure.[188] In this case, the refractive properties are changed and light is absorbed in the structure due to several internal reflection.[189] Up to now, carbon nanotubes have been an ideal candidate for constructing nano-scale structure, which shows a high emissivity of 0.99 with a broadband of wavelengths.[184] Align structure is also applicable for carbon nanotubes and an array with $2 \mu \mathrm{m}$ thickness and lower than $2 \%$ reflection rate was achieved. Hierarchical structure such as graphene foam was also utilized to enhance the light absorption and reduce reflection as light absorber. The graphene foam shows high light absorption over a broad range of solar 
spectrum with ultralow reflection, which enabled highly efficient light-to-heat conversion of over $90 \% \cdot[51]$

Another state-of-the-art light absorber technique utilizes the plasmonic effect of metals. These metals are capable of free-carrier absorption, which is due to the high concentration of free carriers.[190] However, due to the opaque nature of metals, the plasmonic effect only happen at the surface of the materials, which limit its solar-to-heat conversion. In this regard, metal nanoparticles, such as platinum, gold and silver, are fabricated to maximize the heat generation by increasing the surface-to-volume ratio of materials. The electrons on the surface of the metal change from lower energy level to higher ones when exposed to incident light, which creates hot electrons. Afterwards, the harvested energy is transformed into thermal energy because of the electron-phonon scattering and the heat is released due to phonon-phonon relaxation. Therefore, the metals with localized surface-plasmon resonances are optimal to transform photon energy thermal energy. One of the major drawbacks of plasmonic metal as light absorber is the narrow inherent absorption spectra.[153]

\subsubsection{Heat Management in Light Absorbers}

Aside from the conversion efficiency of light to heat, the transfer efficiency of the obtained heat also has an impact on the energy efficiency of the whole system. Heat is transferred in three routes: radiation, convection and conduction. Radiation entails no direct contact because heat was transferred via electromagnetic waves; convection occurs in fluids; while conduction involves direct contact with the heat source and the target. All materials with an absolute temperature $>0 \mathrm{~K}$ emit radiation. Therefore, every material can absorb and emit solar energy at the same time. The radiation spectrum of a material is not related to its intrinsic material properties including shape, structure or size, according to Planck's law.[191] The radiation 
spectrum shifts to shorter wavelengths when the temperature increases. Within a solar vapor generation system, the energy converted from light can be categorized into three types: energy lost from the conversion process; energy used for the water evaporation; and conduction and conversion heat loss to the environment. The higher the energy is used to power the water evaporation, the more efficient the system is. In this regard, heat management is highly sought after to increase the energy efficiency of the SVG aside from structural design of the materials.[192]

Volumetric heating strategy was developed to increase the energy efficiency of the SVG system by increasing the heat transferred to water. In this strategy, the light absorber was uniformly distributed and in direct contact with water, carrying out in situ heating of the water. The drawback of this method is the occurrence site of the evaporation. The evaporation is located at the surface of the water-air interface, whereas the heat is generated at the bulk of water, leading to energy waste due to convection. Moreover, the material is prone to aggregation and deterioration, which is not suitable for long-term application.[193]

To remediate this issue, nanostructures were developed to reduce energy loss by utilizing a water-in-absorber strategy, which is named as interfacial SVG system. In this strategy, water molecules are confined in the pores, gaps or channels of the nanostructure light absorber, which is floating at the surface of the water-air interface where the heating is highly localized and it is easy for evaporated water molecules to escape.[194] The efficiency can reach $90 \%$ with several common materials including wood, carbon black and graphene.[50, 195-197]

Aside from the nanostructured materials, hydrophilic polymers have been developed applying the same strategy to increase the water distribution in the light absorber. In the polymeric network, water molecules are trapped in-between the hydrophilic polymer chains, which forms 
hydrogels.[198] The absorbed energy from sun light can be easily transferred to the water molecules due to the close proximity of light absorber and water. The degree of crosslinking can be tuned to change the pore size of the hydrogel, hence determining the amount of water in the hydrogel. A recently reported hydrogel based SVG can convert sun light to heat with an overall efficiency of 93\% utilizing PVA as the polymer network.[199]

\subsubsection{Conclusion}

In contrast with the current distillation method that utilizes fossil fuel, SVG systems are considerably eco-friendly. The SVG only utilizes the abundant resource of solar energy for water distillation, largely reduces the dependency of cumbersome infrastructure and traditional fuel. In addition, the state-of-the-art SVG systems are capable of water evaporation with lower than 1 sun irradiation, making future disaster relief, household freshwater supplement possible.[200] Current SVG systems are still in the small-scale portable application stage, filling in the blanks of centralized water purification. The scalability and high cost demand of SVG systems are the main drawbacks that hold the technology develop beyond laboratory settings. These problems can be addressed by developing more low-cost, stable nanostructured materials for industrial application. Moreover, wastewater is often complex in its composition, including corrosive chemicals and contaminants. The SVG systems are more focused on using seawater as the resource for water purification, whereas more complicated wastewater treatment is largely ignored. Thus, stability and robustness of materials used for SVG should be considered to increase the durability as well as the versatility of the system.

In summary, SVG is a novel technology that is in its early development stage, which holds great potential for industrial application if the above issues are solved. More innovation of materials and studies on thermal-loss minimization, compact installation will largely improve the 
development of SVG technology. In the future, SVG systems with multi-functionality of wastewater collection, water purification and freshwater delivery will be of great demand for practical solar-powered freshwater purification. 


\section{Chapter 3 Dual-functional Superwettable}

\section{Nanofibrous Membrane}

\subsection{Introduction}

The rapid industrialization has given rise to a severe deterioration in water quality[201]. Given that $70 \%$ of the drinking water comes from rivers and streams, the industrial contamination of these resources can bring about fatal damage to human health[202]. The wastewater emitted from industrial processes, partially comprised of immiscible organic solvents/water mixture, has tremendously increased the difficulty of water treatment[203]. Therefore, developing an effective water purification process has drawn academic interest and industrial effort to address the issue of clean water crisis.

As one of the solutions, much effort has been devoted to oil/water separation of wastewater discharged from manufacturing processes[62, 204-209]. More recently, super-wettable materials with distinct opposite affinities toward oil or water have been emerging in oil-water separation[210-212].

Herein, we demonstrate a hierarchical nano-structured water purification membrane fabricated with PVA nanofibers and PPy nanoparticles to achieve efficient emulsion separation with low oil-adhesion property as well as high-rate seawater desalination. GO, as one of the most important derivatives of graphene, is valued for its non-toxicity, excellent dispersity and has long been used as reinforcement elements to strengthen the material. Therefore, $0.2 \%$ graphene oxide was mixed into the PVA electrospinning solution as a reinforcement to strengthen the material. 
The nanofibrous PVA membrane (NPM) exhibits the integrated properties of mechanical robustness, superhydrophilic/underwater superoleophobic wettability and broadband solar absorption. The crosslinked electrospun PVA nanofibrous mat acts as a porous skeleton with fine flexibility and internal gaps, leading to a high permeate flux during oil/water separation. The PPy nanoparticles deposited on the surface of the PVA nanofibers in a close-packed fashion, giving the membrane a rough surface with superhydrophilicity and underwater superoleophobicity $\left(158^{\circ}\right)$ and an extremely low oil-adhesion property. The ultra-porous structure with superwettability enables the membrane with the separation ability of surfactant-stabilized immiscible mixtures, with high separation efficiency (oil residue after one-time separation no more than $0.01 \mathrm{wt} \%$ ) and high flux. Unlike many membranes using oleophilic polymer[213, 214] as the substrate, PVA is less likely to cause oil fouling because of its intrinsic hydrophilicity. The PPy coated on the surface of PVA further creates a hierarchical structure and superhydrophilic chemistry that act as an oil-repellent barrier, thus enhancing the anti-fouling performance. The NPM exhibited a 98\% flux recovery ratio after continuous cyclic separation of surfactantstabilized oil-in-water emulsion. On the other hand, solar steam generation was realized by placing an NPM on the seawater-air interface with solar irradiation. Owing to the high area-tovolume ratio of nanofibers and the high energy conversion efficiency of PPy, the densely packed PPy nanoparticles on the PVA fibers can quickly harvest the solar light and convert it into thermal energy. Furthermore, the hydrophilic nature of PVA helps efficient transportation of water owing to capillary effect, sustaining a continuous water supply for steam generation. The efficient solar energy absorption was verified by an over $99 \%$ light absorption of the material within a broadband wavelength (250 to $1100 \mathrm{~nm})$. A water evaporation rate of $2.87 \mathrm{~kg} \mathrm{~m}^{-2} \mathrm{~h}^{-1}$ was realized with one sun irradiation. To verify the practicality of the membrane, a solar steam 
generator was installed and utilized to collect purified water under natural sunlight and a freshwater collection capability of solar water purification yield of $14.31 \mathrm{~m}^{-2}$ daily. The oilwater separation and desalination performance of the NPM is compared with other related materials (Table 3-1); the dual-functional NPM not only achieved a remarkably separation efficiency (>99.99\%) but also an excellent desalination performance (with the evaporation rate of $2.87 \mathrm{~kg} \mathrm{~m}^{-2} \mathrm{~h}^{-1}$ ). The dual-functional superwetting membrane represents a new approach to more effective purification of clean, safe drinkable water from any source, whether from the ocean or contaminated industrial supplies. 


\begin{tabular}{|c|c|c|c|c|c|c|}
\hline Material & Function & $\begin{array}{l}\text { Separation } \\
\text { capacity }\end{array}$ & $\begin{array}{l}\text { Evaporation } \\
\text { Rate }\left(\mathbf{k g ~ m}^{-2}\right. \\
\left.\quad \mathbf{h}^{-1}\right)\end{array}$ & Price & Scalability & Reference \\
\hline $\begin{array}{l}\text { Dual-functional nano- } \\
\text { structure NPM }\end{array}$ & $\begin{array}{l}\text { Oil/water } \\
\text { separation \& solar } \\
\text { steam generation }\end{array}$ & $\begin{array}{l}>99.99 \% \text { with } \\
\text { surfactant- } \\
\text { stabilized } \\
\text { emulsion }\end{array}$ & $\begin{array}{l}2.87 \text { under } 1 \\
\text { sun }\end{array}$ & Low & Large & This work \\
\hline $\begin{array}{l}\text { Nanoporous copper foam } \\
\text { decorated with carbon black } \\
\text { nanoparticles }\end{array}$ & $\begin{array}{l}\text { Solar vapor } \\
\text { generation }\end{array}$ & - & $\begin{array}{l}1.31 \text { under } 1 \\
\text { sun }\end{array}$ & High & Small & [215] \\
\hline Porous 3D carbon foams & $\begin{array}{l}\text { Solar vapor } \\
\text { generation }\end{array}$ & - & $\begin{array}{l}1.26 \text { under } 1 \\
\text { sun }\end{array}$ & Low & Small & [216] \\
\hline $\begin{array}{l}\text { One-step melamine-derived } \\
\text { carbon sponges }\end{array}$ & $\begin{array}{l}\text { Solar vapor } \\
\text { generation }\end{array}$ & - & $\begin{array}{l}1.98 \text { under } 1 \\
\text { sun }\end{array}$ & Low & Large & [217] \\
\hline $\begin{array}{l}\mathrm{MnO}_{2} \text { nanowire-rGO } \\
\text { monolithic aerogels }\end{array}$ & $\begin{array}{l}\text { Solar vapor } \\
\text { generation }\end{array}$ & - & $\begin{array}{l}\sim 1.5 \text { under } 1 \\
\text { sun }\end{array}$ & Low & Large & [218] \\
\hline $\begin{array}{l}3 \mathrm{D} \text { polyacrylamide based } \\
\text { aerogel }\end{array}$ & $\begin{array}{l}\text { Solar vapor } \\
\text { generation }\end{array}$ & - & $\begin{array}{l}2.0 \text { under } 1 \\
\text { sun }\end{array}$ & Low & Small & [219] \\
\hline $\begin{array}{l}\mathrm{SiO} 2 / \text { cellulose } \\
\text { nanofiber/carbon nanotube } \\
\text { based Janus evaporator }\end{array}$ & $\begin{array}{l}\text { Solar vapor } \\
\text { generation }\end{array}$ & - & $\begin{array}{l}1.2 \text { under } 1 \\
\text { sun }\end{array}$ & Low & Large & [220] \\
\hline $\begin{array}{l}\mathrm{CuS} \text { yolk-shell nanocage- } \\
\text { agarose aerogel }\end{array}$ & $\begin{array}{l}\text { Solar vapor } \\
\text { generation }\end{array}$ & - & $\begin{array}{l}1.63 \text { under } 1 \\
\text { sun }\end{array}$ & Medium & Small & {$[221]$} \\
\hline $\begin{array}{l}\text { Cupric Phosphate } \\
\text { nanosheets-wrapped } \\
\text { inorganic membrane }\end{array}$ & $\begin{array}{l}\text { Oil/water } \\
\text { separation } \\
\text { membrane }\end{array}$ & $\begin{array}{l}\text { high efficiency } \\
\text { with surfactant- } \\
\text { free mixture }\end{array}$ & - & High & Large & {$[222]$} \\
\hline $\begin{array}{l}\text { Nonflammable } \\
\text { superhydrophobic paper }\end{array}$ & $\begin{array}{l}\text { Oil/water } \\
\text { separation } \\
\text { membrane }\end{array}$ & $\begin{array}{l}99.9 \% \text { with } \\
\text { surfactant-free } \\
\text { mixture }\end{array}$ & - & Low & Low & {$[223]$} \\
\hline $\begin{array}{l}\text { Aliphatic polyketon } \\
\text { composite membranes }\end{array}$ & $\begin{array}{l}\text { Oil/water } \\
\text { separation } \\
\text { membrane }\end{array}$ & Near $100 \%$ & - & High & Low & {$[224]$} \\
\hline $\begin{array}{l}\text { Janus PANI-SiO } \\
\text { nanoparticles-modified } \\
\text { membrane }\end{array}$ & $\begin{array}{l}\text { Oil/water } \\
\text { separation } \\
\text { membrane }\end{array}$ & $>99.7 \%$ & & Medium & Low & {$[225]$} \\
\hline $\begin{array}{l}\text { Ultrathin in situ silicification } \\
\text { layer }\end{array}$ & $\begin{array}{l}\text { Oil/water } \\
\text { separation } \\
\text { membrane }\end{array}$ & $>99.9 \%$ & - & Medium & Medium & [226] \\
\hline $\begin{array}{l}\mathrm{TiO}_{2} \text { modified } \\
\text { superhydrophilic } \\
\text { membrane }\end{array}$ & $\begin{array}{l}\text { Oil/water } \\
\text { separation } \\
\text { membrane }\end{array}$ & $>98 \%$ & - & High & Medium & {$[227]$} \\
\hline $\begin{array}{l}\text { Polydopamine coated cotton } \\
\text { coating }\end{array}$ & $\begin{array}{l}\text { Oil/water } \\
\text { separation } \\
\text { membrane }\end{array}$ & Near $100 \%$ & - & Low & Medium & [228] \\
\hline
\end{tabular}

Table 3-1. The performances of oil water separation and solar steam generation of different materials. 


\subsection{Materials and Methods}

\subsubsection{Materials}

PVA $(\mathrm{Mw}=205,000$, Mowiol $\AA$, Millipore Sigma), glutaraldehyde (GA, 25\% aqueous solution, Alfa Aesar), pyrrole (98\%+, Alfa Aesar) and Iron(III) nitrate (anhydrous, 98\%, Alfa Aesar), hydrochloric acid ( $\mathrm{HCl}, 37 \%$ reagent grade, Sigma Aldrich), GO (Tanfeng Tech. Inc., China), Bovine Serum Albumins (BSA) (Sigma-Aldrich), humic acid (HA) (Millipore Sigma). Deionized water was obtained water from EASYpure II LF ultrapure water system.

\subsubsection{Electrospinning of PVA}

$10 \mathrm{~g}$ of PVA powder was dissolved in $90 \mathrm{~g}$ of DI water under vigorous stirring for $6 \mathrm{~h}$ to prepare $10 \%$ PVA solution. Different concentrations of GO were homogeneously mixed into the PVA solution with vortex mixer. The PVA-GO mixture was added in a $10 \mathrm{ml}$ syringe fitted with $18 \mathrm{G}$ flat end stainless steel needle. Electrospinning of the PVA-GO mixture was set up with a 15-18 KV high voltage and the distance between the tip of the needle and the collector was $165 \mathrm{~mm}$. The PVA solution was pumped out by a syringe pump (PHD2000, Harvard Apparatus) at a flow rate of $5 \mathrm{~mL} / \mathrm{h}$ to achieve a continuous nanofiber deposition and the NPM were formed onto a textiled nickel mesh $(10 \mathrm{~cm} \times 10 \mathrm{~cm})$ as a collector.

\subsubsection{Crosslinking of Electrospun PVA-GO Membrane}

The obtained PVA-GO membranes were instantaneously dissolved in water as PVA has a high swell ratio and fibers can fuse with each other. To stabilize the membrane, the electrospun GOPVA mat was pre-crosslinked with glutaraldehyde vapor. The pre-crosslink was carried out by 
exposure of the as-electrospun PVA-GO mat to with $600 \mu \mathrm{lGA}(2.6 \mathrm{M})$ and $200 \mu 1 \mathrm{HCl}(37 \%)$ in a sealed container for 24 hours. To further stabilize the membrane, the GO-PVA membrane was immersed in $40 \mathrm{ml}$ acetone solution with $500 \mu \mathrm{l} \mathrm{GA}$ for further crosslinking. The originally separate nanofibers merged and entangled among each other and bonding was formed at the intersection points between the nanofibers. Afterwards, the membranes were washed with distilled water for 5 times, before drying under ambient atmosphere. The membrane was referred as NPM1.

\subsubsection{Preparation of NPM5}

The crosslinked PVA-PPy-GO membrane was coated with PPy via in situ polymerization of pyrrole. The crosslinked membrane was soaked in $10 \mathrm{ml}$ solution of $8 \mathrm{wt} \% \mathrm{Fe}(\mathrm{III})$ nitrate for 20 min under $4{ }^{\circ} \mathrm{C}$. Afterwards, another solution containing $340 \mu \mathrm{l}$ of pyrrole dissolved in $10 \mathrm{ml}$ of DI water was added under $4{ }^{\circ} \mathrm{C}$. After polymerization for 3 days, the PPy coated membrane was taken out of the solution, washed with deionized Milli-Q water and dried at ambient atmosphere.

\subsubsection{Characterization}

Field-emission scanning electron microscopy (JEOL, JSM-5900LV, SEM Tech Solution) was conducted at an accelerating voltage of $20 \mathrm{eV}$. All specimens were sputter-coated with gold. FTIR spectroscopy analysis was performed using a Thermo Nicolet iS10 FTIR Spectrometer (Thermo Nicolet, Madison, WI, USA) from 4000 to $400 \mathrm{~cm}^{-1}$ with a resolution of $2 \mathrm{~cm}^{-1}$ and an accumulation of 32 scans. Tensile tests were conducted at room temperature with samples of 20 $\mathrm{mm}$ in length, $5 \mathrm{~mm}$ in width and $200 \mu \mathrm{m}$ in thickness on a tensile testing machine (LS5, Instron, Inc., USA). The membranes were fixed by the grips and subjected to an crosshead speed of 10 
$\mathrm{mm} / \mathrm{min}$ using a gauge length of $20 \mathrm{~mm}$. Tensile test for each group were carried out with 3 specimens. Underwater oil CA and water CA were tested on a goniometer (JY-PHA, Shengding, China). To test the underwater oil contact angle, a $5 \mu 1$ chosen organic solvent or oil was dispensed on a NPM placed in a water-filled petri-dish. To test the dynamic sliding angle of the NPM, a $15 \mu 1$ chosen organic solvent or oil droplet was placed on the membrane and the sliding angle was the minimum tilt angle required for the droplet to move. The anti-fouling capability of the NPM was evaluated by a self-cleaning experiment: a NPM was immersed in canola oil, followed by transferring the NPM to a container filled with DI water. The container was gently shacked to remove the oil from the NPM. Dynamic oil adhesion test was carried out to examine the capability of the NPM to repel oil with high viscosity, in which a $5 \mu$ of highly viscous mechanical pump oil was pressed on the surface of NPM and subsequently detached from the membrane surface.

\subsubsection{Oil/water Emulsion Preparation and Filtration Evaluation}

Surfactant-free oil/water emulsion was prepared by sonicating a $10 \%$ chosen organic solvent-inwater mixture for 10 minutes in water bath. The surfactant-stabilized emulsion was prepared by mixing $0.01 \%$ sodium dodecyl sulfate (SDS) into $500 \mathrm{ml}$ of DI water, followed by adding $50 \mathrm{ml}$ chosen organic solvent or oil into the solution. A homogenized emulsion was obtained after sonicating the oil/surfactant/water mixture for $2 \mathrm{~h}$ in water bath. The organic solvent/oil droplet size was measure by a dynamic light scattering machine (Zetasizer, Nano-ZS, Malvern, UK) and optical microscopy (Micromaster, Fisher Scientific).

For the fouling resistant testing, DI water was first poured into the filtration equipment and the flux was recorded as $F_{1}$. After 10 min of pure water filtration, oil/water emulsion was poured into 
the filtration equipment for 60 min of continuous filtration. The NPM was subsequently released from the equipment and was with DI water to remove the oil residue. The flux of pure water was tested again with the same NPM and the flux was recorded as $F_{2}$. The anti-fouling capability of the NPM was measured by the flux recovery ratio (FRR) according to the following equation:

$$
F R R=\frac{F_{2}}{F_{1}} \times 100 \%
$$

The separation efficiency of the NPM was calculated by comparing the oil droplet count before $\left(\mathrm{C}_{\mathrm{o}}\right)$ and after $\left(\mathrm{C}_{\mathrm{p}}\right)$ filtration according to the following equation:

$$
R(\%)=\left(1-\frac{c_{p}}{C_{o}}\right) \times 100 \%
$$

\subsubsection{Solar Vapor Generation Experiment}

A sun tester (Suntest XLS+, ATLAS) was utilized to test out the solar vapor generation performance of NPM. The solar irradiance was set as $1 \mathrm{~kW} \mathrm{~m}^{-2}$. The NPM5 sample was cut into a round shape with diameter of $8 \mathrm{~cm}^{-2}$. A NPM5 ( 200 $\mu \mathrm{m}$ in thickness) was placed and floated on simulated seawater water (or DI water as control group) in a beaker. The set-up was located in the beam spot with various solar irradiance. A weight balance was utilized to measure the water evaporation performance. Prior to radiation of the experimental set-up, the evaporation rate without any light source was calculated with the beaker placed in dark environment for $1 \mathrm{~h}$. The evaporation rate without any light source was deducted from the solar irradiated evaporation rate. All solar vapor generation rates were calculated after reaching equilibrium state under $1 \mathrm{~kW} \mathrm{~m}^{-2}$ solar irradiance for $1 \mathrm{~h}$. 


\subsubsection{COMSOL Heat Transfer Simulation}

Boundary conditions: 1) input solar energy $\left(1000 \mathrm{~W} \mathrm{~m}^{-2}\right)$, 2) temperature of the underlying bulk water, and 3) temperature of evaporating surface. Parameters: 1) the surface emissivity of 0.95 was used on absorber, 2) A natural convection heat transfer coefficient of $5 \mathrm{~W} / \mathrm{m}^{2} \mathrm{~K}$ was used on evaporation surfaces. 3) The evaporation heat transfer coefficient of the evaporating surface was fit to match the boundary conditions. 4) The parameters of porous materials were based on the water because the materials were filled with large amount of water. (the thermal conductivity $0.6 \mathrm{~W} \mathrm{~m}^{-2} \mathrm{~K}^{-1}$, specific heat $4.2 \mathrm{~J} \mathrm{~g}^{-1} \mathrm{~K}^{-1}$, and density $1000 \mathrm{~kg} \mathrm{~m}^{-3}$ ). 


\section{Chapter 4 Membrane Characterization}

\subsection{Introduction}

In this chapter, the effect of PPy on the NPM was elaborated through various characterization methodologies. The morphologies of the NPM with various degrees of PPy nanoparticle deposition was observed through SEM images. Tensile tests of different degrees of PPy coating were conducted to study the effect of PPy on the mechanical properties of the membrane. Oil and underwater contact angle tests were carried out to study the impact of PPy on the wettability of the membrane. The initial intent of incorporating PPy in the NPM is two-folded. Firstly, PPy may act as a mechanical reinforcement component in the membrane. During coating of PPy, PPy is deposited and aggregated onto the PVA scaffold by strong hydrogen bonding interaction. Theoretically, the overall Young's modulus of the membrane is increased because of the aggregation and deposition of PPy nanoparticles on the PVA scaffold without completely sacrificing the flexibility of PVA. Secondly, the morphology of PPy should play a critical role in the superhydrophilic/superoleophobic property of the membrane. Theoraticlly, PPy nanoparticle is should largely roughen the surface of the PVA nanofibers, which should further contribute to the underwater superoleophobicity of the membrane. For emulsified oil/water filtration process, adhesion of the oil phase on the membrane surface is the main reason for deterioration of wetting selectivity. Therefore, low adhesion of oil on the membrane is an important factor for long-term oil-in-water emulsion separation with high selectivity and efficiency. Underwater oil sliding angle tests were conducted to evaluate the impact of PPy on the underwater oil adhesion towards NPM. The dynamic oil adhesion test was conducted to test the adhesion between the oil droplets and the NPM. 


\subsection{Hierarchical Nanostructure of the Membrane}

To prepare the NPM (Figure 4-1), the electrospun PVA membrane was first pre-crosslinked with glutaraldehyde vapor rather than solution, because the as-electrospun PVA nanofibers are dissolvable in glutaraldehyde water solution (Figure 4-2). After 24 hours of vapor treatment, the pre-crosslinked PVA membrane was processed with liquid phase crosslinking in glutaraldehyde acetone solution to further crosslink the PVA nanofibers. Until now, the PVA membrane was fully crosslinked and capable of keeping the integrity under water (referred as NPM1). Afterward, the membrane was dipped in a mixture of $\mathrm{Fe}^{3+}$ and pyrrole monomer solution. With the process of the slow oxidation of the pyrrole monomers under $4^{\circ} \mathrm{C}$, the scale of the PPy nanoparticle layer on the surface was controlled to be much smaller than the PVA nanofibers to construct a hierarchical nanostructure. The coated PPy nanoparticles significantly roughen the surface and enable the membrane with superhydrophilicity/superolephobicity, which plays a critical role in the separation of surfactant-stabilized immiscible oil/water mixture as well as fouling-resistant property. As can be seen from Figure 4-2b, the entangled PVA fibers form a porous interconnected 3D network with irregular interstice. After the deposition of in situ synthesized PPy on the PVA nanofiber skeleton, the membrane turned pitch black (Figure 4-2d) and clusters of particles were observed in between the internal gaps (Figure 4-2e). The enlarged SEM illustration of the membrane surface (Figure 4-2f) shows that the added PPy roughened the surface of PVA nanofibers by forming tightly packed nanoscale particles, which is different from the smooth as-electrospun PVA fibers without PPy deposition (Figure 4-2c). These finer particles are randomly deposited on the surface of nanofibers while closely attached with each other, as a result forming a fairly coarse morphology, which explains the underwater superoleophobicity according to the Cassie's model in Young's equation[85]. The micro- and 
nano-scale PPy particles play a critical role as light absorber with low reflectance because of the dark appearance of PPy and the effect of PPy will be discussed later.
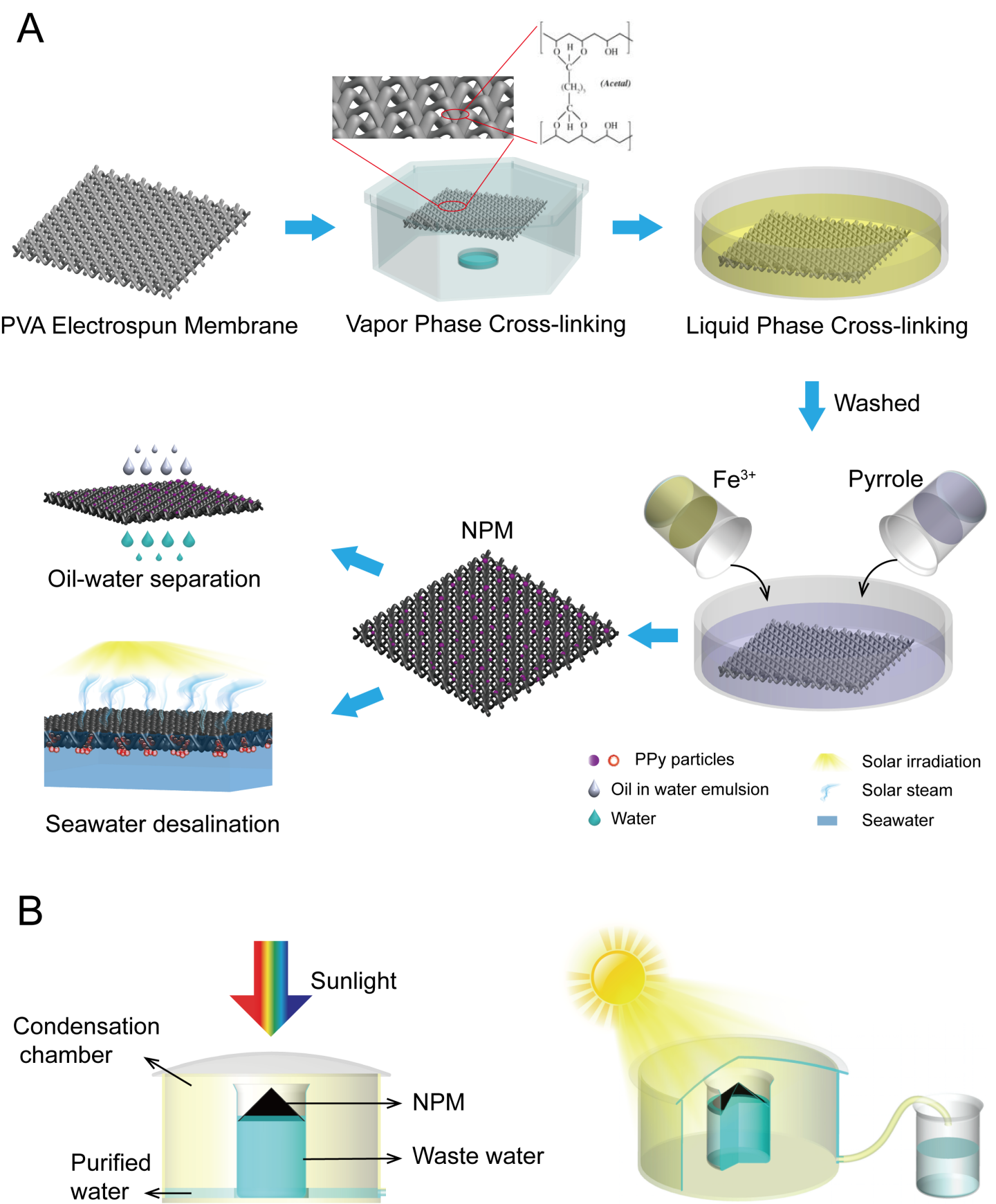

Figure 4-1. (a) Schematic Illustration of the preparation process of the NPM membrane and the dual functions of the membrane. (b) the on-site set-up illustration for seawater desalination. 
(a) Without crosslinking

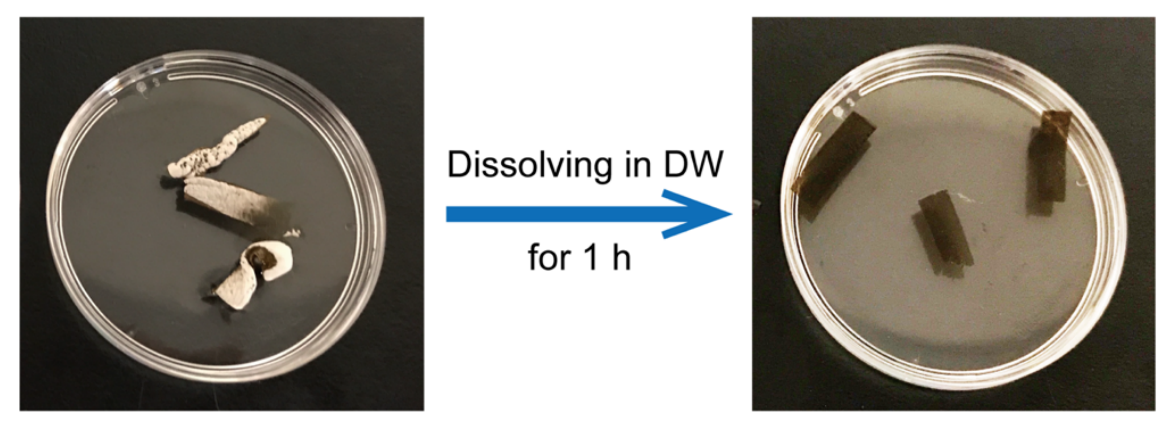

(b) Crosslinked by glutaraldehyde
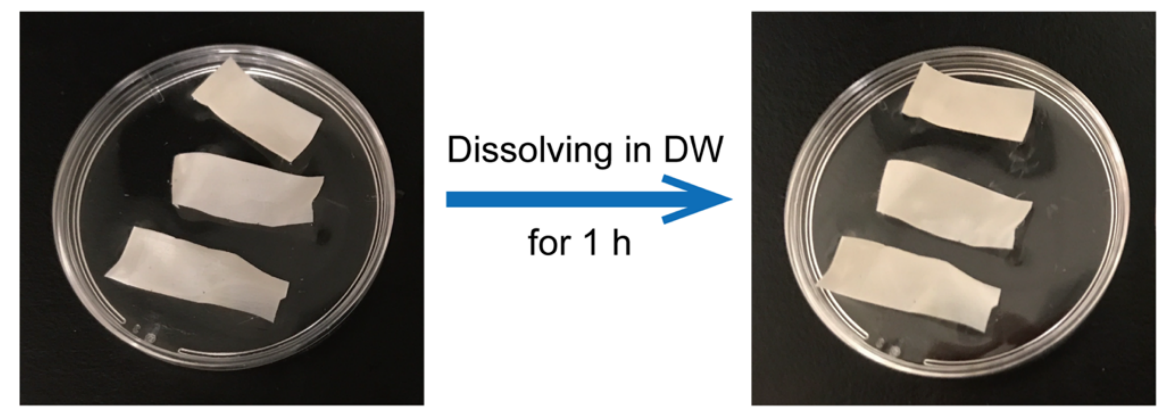

Figure 4-2. Photographs of PVA membranes without (up) and with (down) crosslinking of 25\% glutaraldehyde after dissolving in distilled water (DW) for $1 \mathrm{~h}$. 

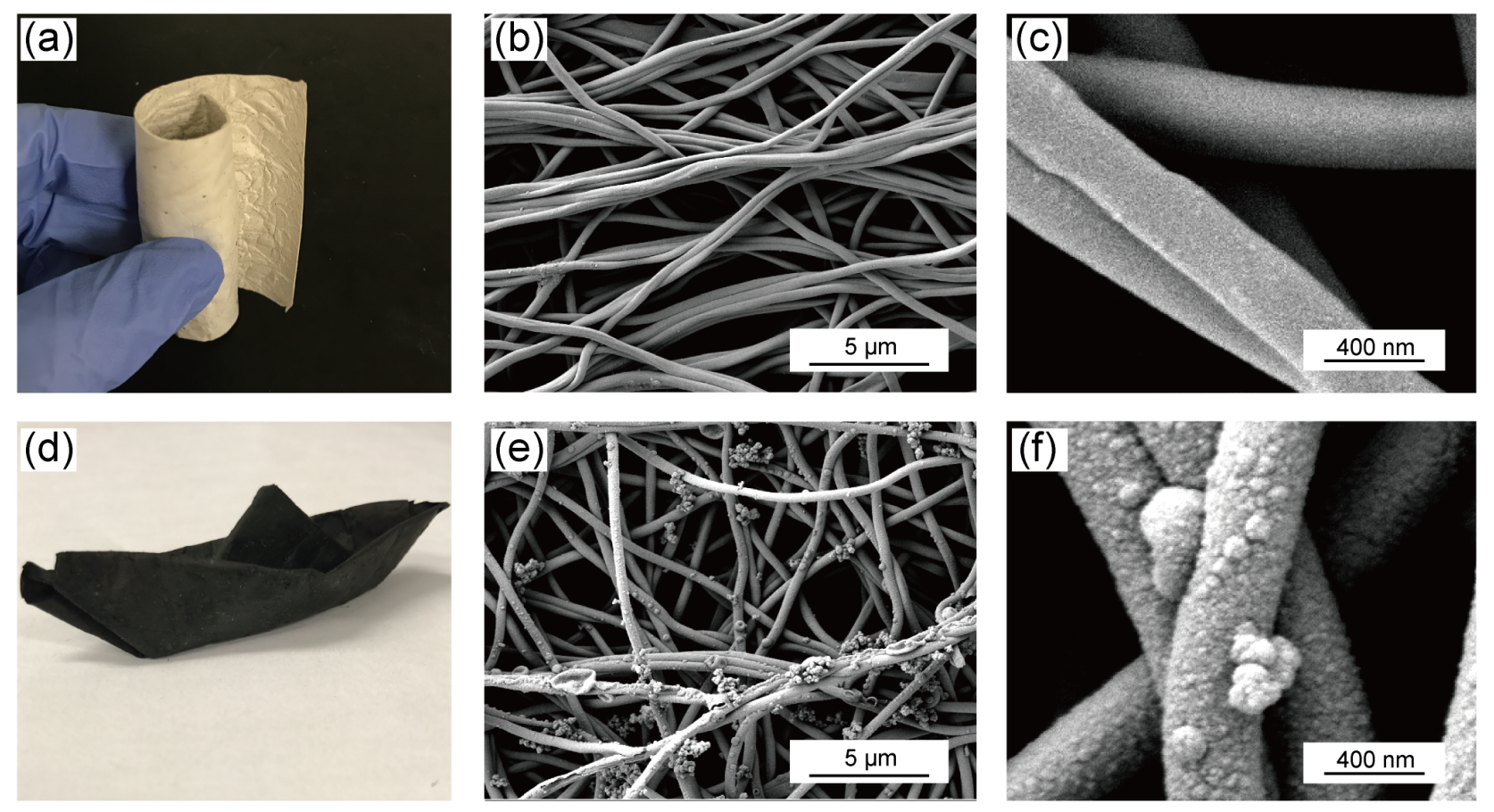

(g)

(h)
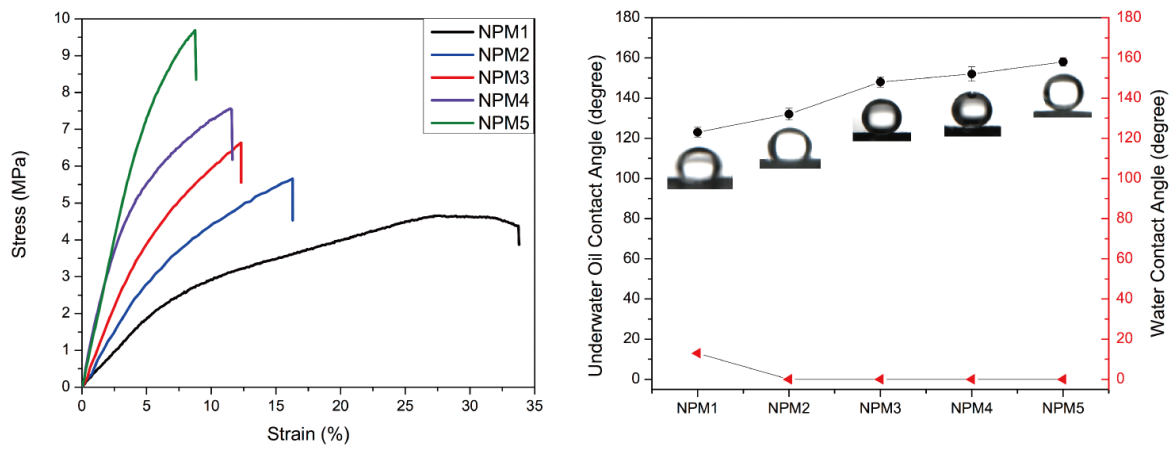

(i)

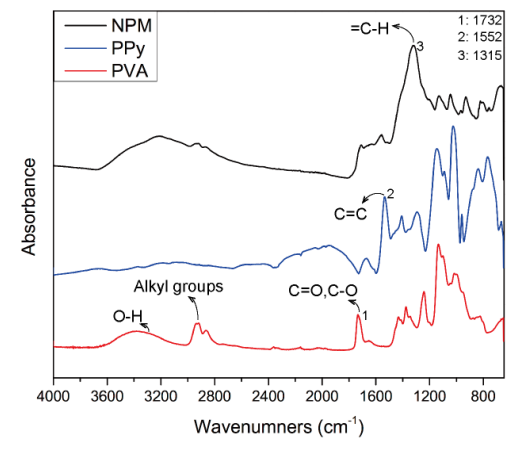

Figure 4-3. Surface morphological changes of the NPM, tensile stress versus strain curves of the crosslinked of the NPM, contact angles and FT-IR spectrum of NPMs. 


\subsection{Mechanical Property of the NPM}

Given that the membrane is designed to endure repeated oil/water separation and seawater desalination, the durability of the membrane in aqueous condition is a crucial factor to be considered. The mechanical property of the membrane was modified by adding GO during electrospinning considering its high young's modulus and strong mechanical property. The stress and strain behavior of NPM was detailed in Figure 4-4. The ratio of GO and PVA was investigated to understand its effect on the mechanical properties of the membrane. As presented in Figure 4-4, the mechanical properties of the NPM can be adjusted with a various ratio of the reinforcement (GO) and matrix (PVA). As predicted, by changing the ratio of GO to PVA from 0 to $0.3 \%$ led to the decrease of elongation from $60.07 \%$ to $17.14 \%$ and an increase of modulus from 10.42 MPa to $114.36 \mathrm{MPa}$. With a decrease of the GO/PVA ratio, the tensile strength of the nanofibers initially increased. At the $0.3 \%$ ratio of GO/PVA, the nanofiber membranes exhibited the ultimate tensile strength of $6.08 \mathrm{MPa}$ and the strain at break of $17.14 \%$. However, the high ratio of GO/PVA results in brittleness in mechanical property, which is undesirable in the separation. Given that PPy will further strengthen the material, $0.2 \%$ GO/PVA was chosen as an optimal amount which possesses high strength but also a reasonable flexibility. 


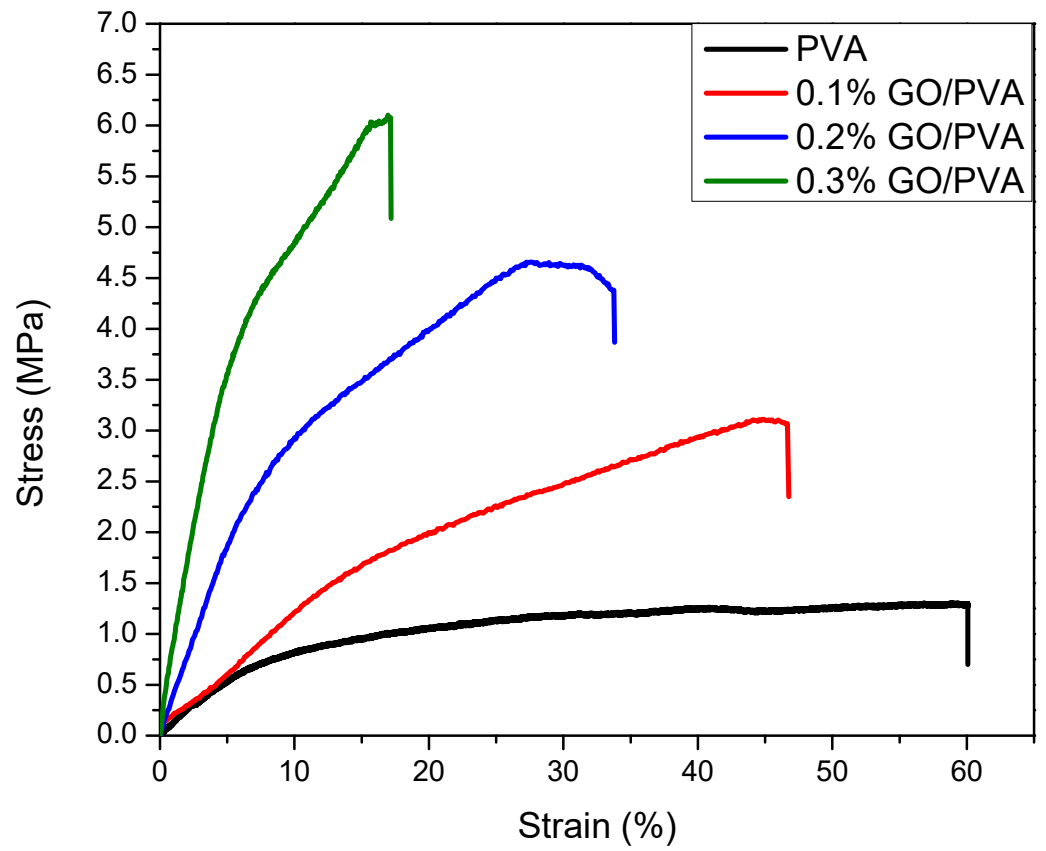

Figure 4-4. Tensile stress strain curves of the crosslinked PVA membranes mixed with $0.1 \%$, $0.2 \%, 0.3 \%$ GO and no $G O$. 
To clarify the influence of PPy on mechanical properties and wetting behavior of the membrane, tensile test and underwater contact angle test were carried out with various PPy content. Figure 4-3g shows typical stress-strains curve of the NPM with different extent of PPy coating. The mechanical property of NPM is tunable via changing the proportion of rigid constituent (PPy) and flexible constituent (PVA). The crosslinked PVA membranes(NPM1) are immersed in pyrrole monomer solution for 5 hours (NPM2), 1 day (NPM3), 2 days (NPM4) and 3 days (NPM5), respectively. As expected, with the PPy in situ polymerization proceeding, more PPy particles were aggregated on PVA nanofibers (Figure 4-5), resulting in the elongation decrease from $33.75 \%$ to $8.75 \%$ and the modulus increase from $41.51 \mathrm{MPa}$ to $159.37 \mathrm{MPa}$. The ultimate strength of NPM achieved 7.62 MPa after 2 days of PPy coating. With the slow oxidation of PPy reacting for 3 days, the NPM5 shows the highest strength at break of $9.69 \mathrm{MPa}$ and a Young's modulus of $159.37 \mathrm{MPa}$ with an elongation of $8.75 \%$, comparing to the NPM1 with lower modulus (10.42 MPa) and higher flexibility (fracture strain at 33.75\%). 

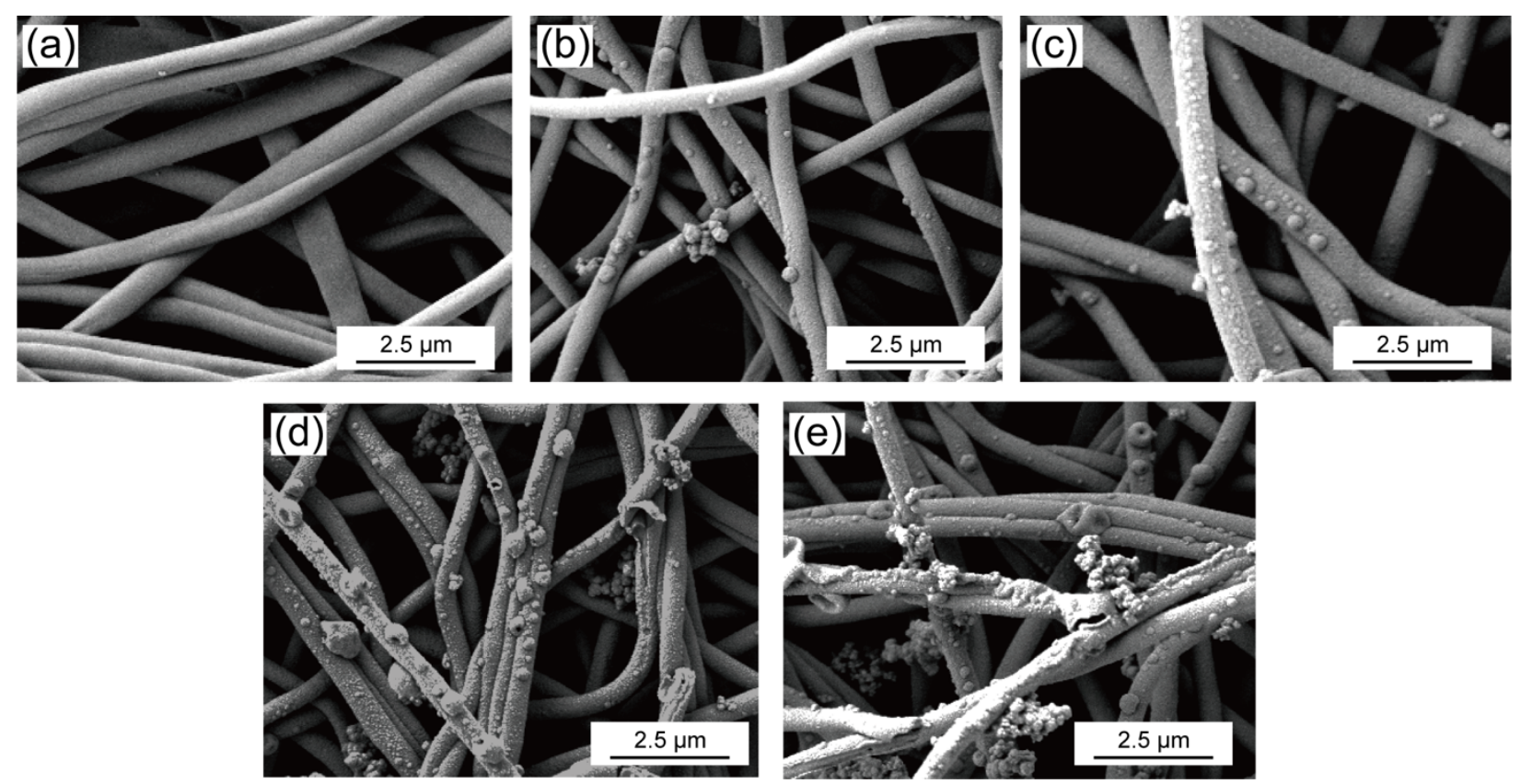

Figure 4-5. SEM images NPM1 to NPM5. 


\subsection{Water and Underwater Oil Contact Angle Tests}

A hypothesis was raised that the surface wettability is directly influenced by the amount of PPy coated onto PVA nanofibers. Therefore, the effect of PPy on the wetting behavior of membranes was also investigated. As a result, the roughness of the surface of the NPM, tightly related to the deposition of PPy nanoparticles on the PVA nanofibers, has great impact on the water or oil wettability of the material. As can be observed in Figure 4-3h, NPM1 exhibits a water CA of $13^{\circ}$ and an underwater oil CA of $123^{\circ}$. After immersing the membrane in PPy solution for $5 \mathrm{~h}$, the water $\mathrm{CA}$ reduces to $0^{\circ}$ and the underwater oil CA increases to $132^{\circ}$. With continuous immersion for 2 days, more PPy nanoparticles are synthesized and deposited onto the surface of the PVA nanofibers, leading to higher hydrophilicity and oleophobicity with an underwater oil contact angle of $148^{\circ}$ after 1 day. After 2 days, the water contact angle stays at $0^{\circ}$ while the underwater oil contact angle increases to approximately $152^{\circ}$. Once the membrane was immersed in PPy for 3 days, water contact angle still remains a $0^{\circ}$ and an average of $158^{\circ}$ underwater oil contact angle is observed, demonstrating superhydrophilicity and underwater superoleophobicity. This phenomenon is in accordance with the hypothesis that the deviation of wettability is closely associated with the amount of PPy nanoparticles packed on the PVA nanofibers surfaces. The result can be explained by Wenzel's model[61], where increased ratio of actual to geometric surface area results in higher roughness factor, which brings about higher affinity toward oil or water. Therefore, as for a particle-composed surface, more nanoparticles bring about a greater coarseness, thus gives the membrane a superhydrophilic wettability. The detailed mechanism will be presented in the following discussion.

To verify whether PPy nanoparticles are successfully deposited onto the PVA fibers, the FT-IR 
spectrum of the NPM was analyzed (Figure 4-3i). As can be observed in the PVA spectrum (red line in Figure 4-3i), the broad band of PVA $\left(3550-3200 \mathrm{~cm}^{-1}\right)$ is attributed to O-H stretching from the hydrogen bond. The presence of alkyl groups in PVA results in vibrational band between 3000 and $2850 \mathrm{~cm}^{-1}$. The characteristic peak around $1732 \mathrm{~cm}^{-1}$ is attributed to the $\mathrm{C}=\mathrm{O}$ and C-O stretches in the acetate groups[229]. As can be seen from the PPy spectrum (Figure 4-3i, blue), absorption at $1,552 \mathrm{~cm}^{-1}$ is attributed to the in-ring stretching of $\mathrm{C}=\mathrm{C}$ bonds in the pyrrole ring[230], while the absorption at $1,315 \mathrm{~cm}^{-1}$ is associated with in-plane vibration of $=\mathrm{C}-\mathrm{H}$ bonds[231]. As can be seen in the black curve in Figure 4-3i, most of the characteristic absorptions of PPy and PVA described above were observed in the FT-IR spectrum of NPM5 without shifts, therefore confirming the existence of PPy and PVA in the membrane. 


\subsection{Organic Solvent Contact Angle and Sliding Angle Test}

Aside from oil, the wetting behaviors of the membrane with several non-polar organic solvents were also investigated. In under water contact angle test (Figure 4-6a), the process of the water droplet being completely soaked by the membrane took less than $0.5 \mathrm{~s}$. Whereas the membrane resisted the adhesion of organic solvents even after repeated contact (Figure 4-6b), indicating the low adhesion force between NPM and organic solvents, which can be explained by its rough nano-structured surface that allows for the approaching oils being repelled by the soaked water. The underwater oil contact angles of canola oil and several versatile organic solvents, including hexane, toluene, petroleum ether are all above $154^{\circ}$ (Figure 4-6e). Wettability test of the membrane using non-soluble organic solvents with densities higher than water (i.e. chloroform, dichloromethane and carbon disulfide, excellent underwater superoleophobicity were also carried out, with underwater oil contact angle of over $156^{\circ}$. The stability of this wetting behavior was tested by immersing NPM5 in water for 30 consecutive days. As a result, the membrane maintained its underwater superoleophobic property, suggesting its exceptional durability under aqueous environment (Figure 4-7). The underwater superoleophobicity also suggests that the NPM potentially possesses anti-fouling propensity due to its superior surface hydration and low adhesion force towards oil. To further investigate the oil-repellent property of NPM, sliding angle tests were carried out. It can be observed from Figure 4-6c that the dichloromethane droplet easily rolled off the surface of NPM5 with a SA of $\sim 1^{\circ}$. In contrast, the dynamic SA dichloromethane on NPM1 is $\sim 10^{\circ}$ (Figure 4-8) due to the lack of PPy nanoparticles on the PVA nanofibers. The hydrophilicity of NPM was enhanced by the presence of PPy nanoparticles because of the synergistic effect of surface energy and the nanoscale topography. The dynamic sliding angles of various organic solvents on NPM5 were comprehensively studied, as shown in 
Figure 4-6f. The sliding angles (SA) ranges from $1^{\circ}$ (chloroform and dichloromethane) to $3^{\circ}$ (canola oil), showing outstanding underwater superoleophobicity. 

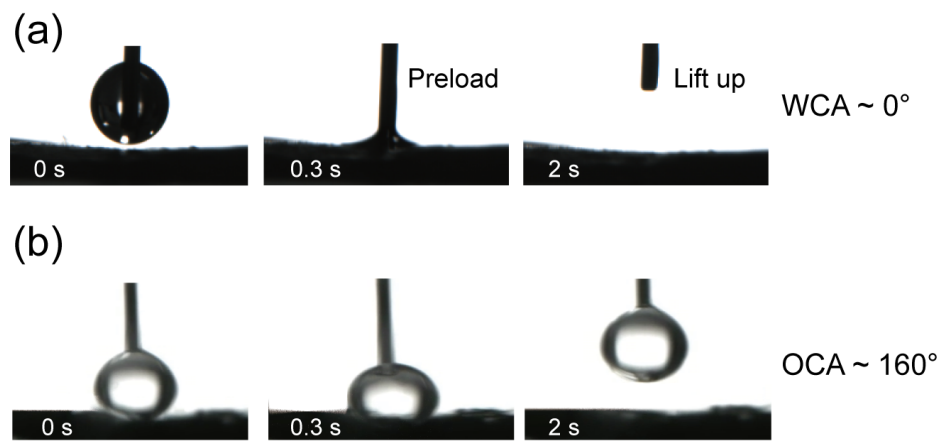

(d)

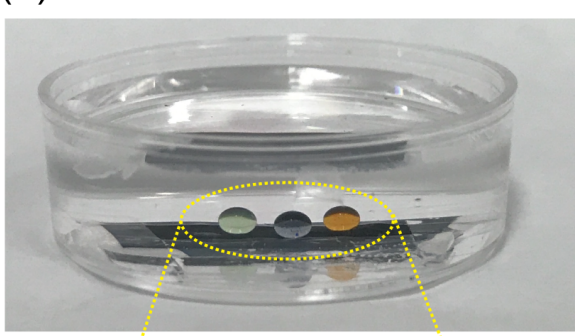

(c)
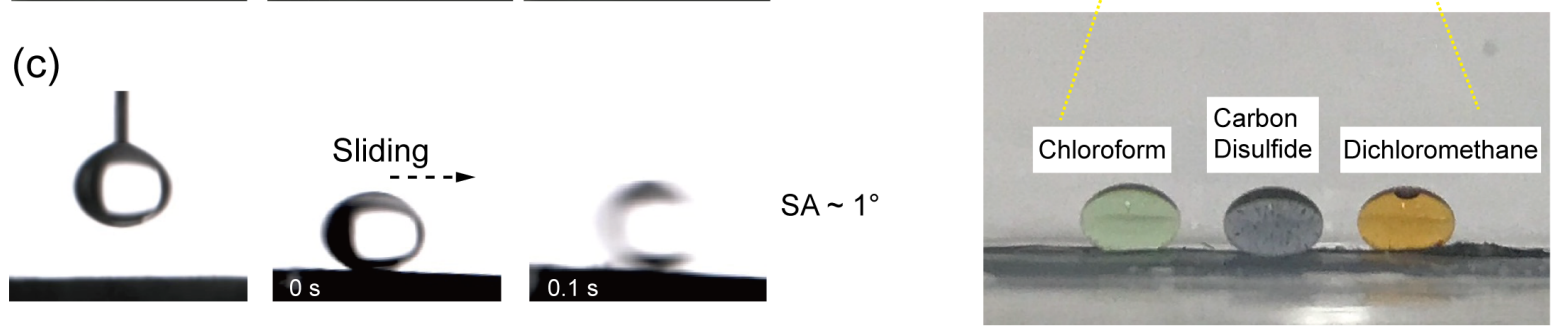

(e)

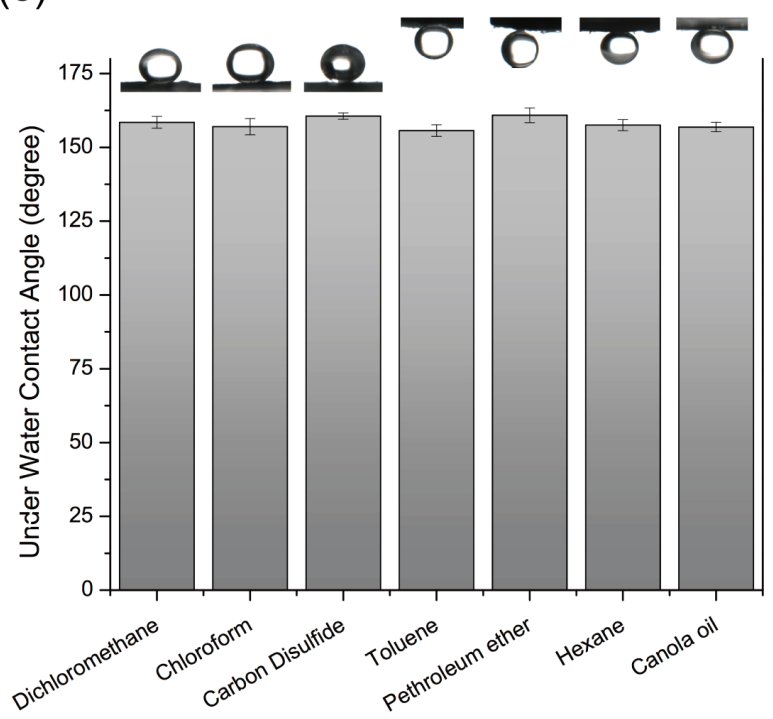

(f)

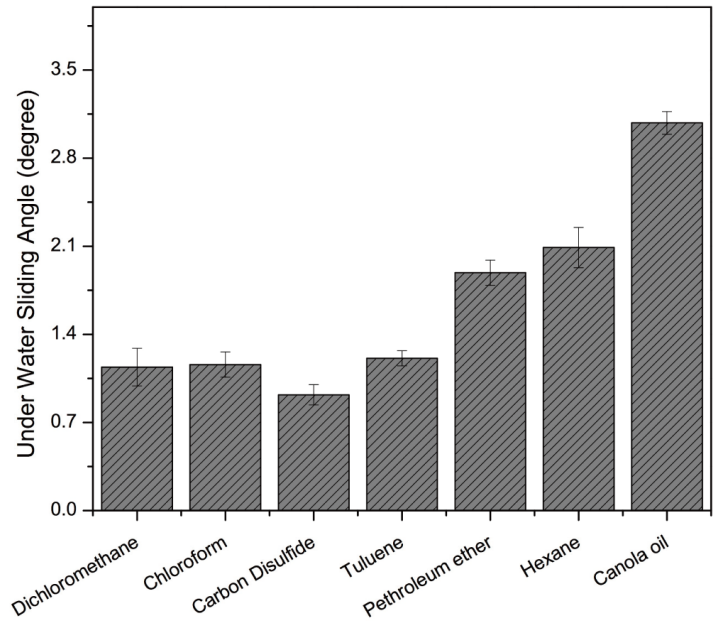

Figure 4-6. Underwater Oil Contact Angles and underwater sliding Angles of the NPM against different oily droplets. 


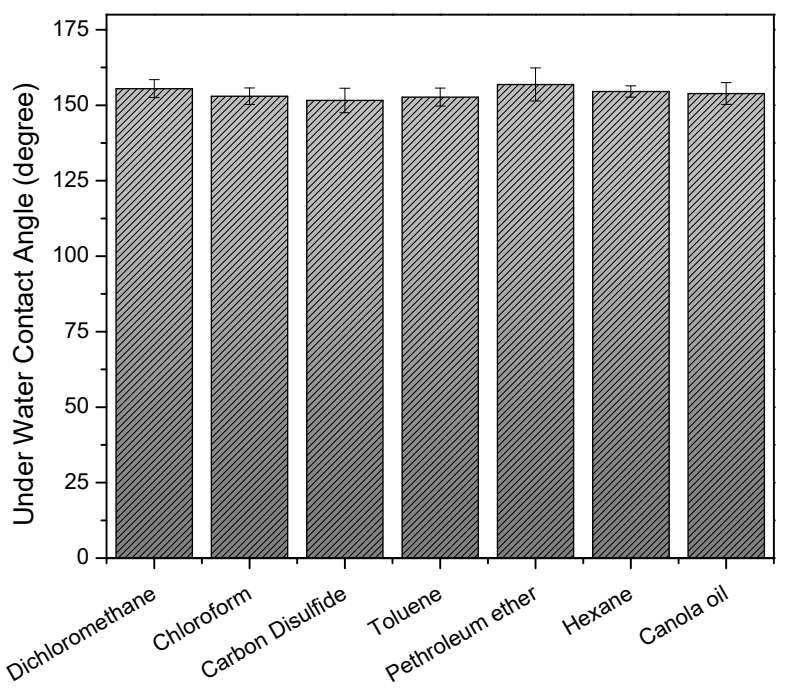

Figure 4-7. Underwater Oil Contact Angles of the NPM against different oily droplets after immersing the membrane in artificial seawater for 30 consecutive days.

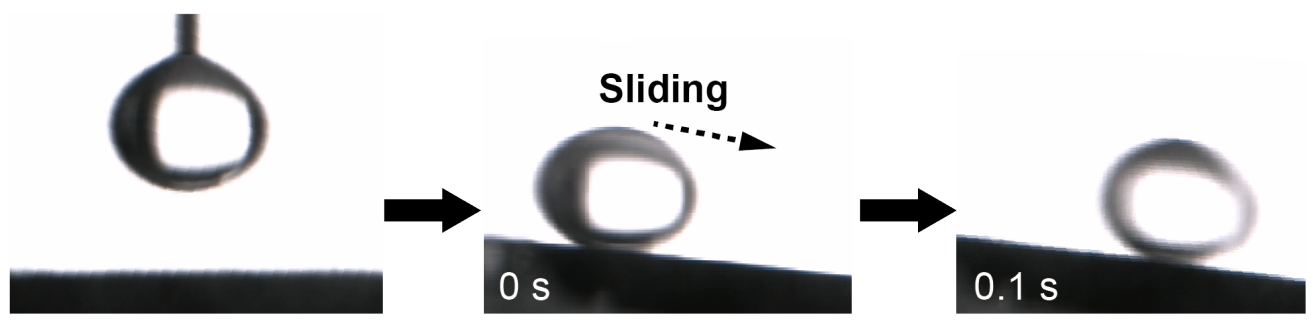

Figure 4-8. Dynamic sliding angle test of NPM1 with a droplet of dichloromethane 


\section{Chapter 5 Oil/water Separation Performance}

\section{of the NPM}

\subsection{Introduction}

This chapter will discuss the Oil/waters separation performance of the NPM under various separation conditions. Non-polar organic solvents that share the non-solubility property of oil were used to test the separation efficiency of the NPM. Six insoluble organic solvents that are commonly used in industrial setting was chosen for the testing. The effect of surfactant on separation process by emulsifying the oil/water mixture as stabilized emulsion for separation. The separation permeation flux of surfactant-free and surfactant stabilized emulsions were recorded, as with the separation efficiencies. The oil droplets sizes were measured by DLS. Ability to operate under harsh conditions is another factor to ensure reliable and long-term separation. Therefore, acid/alkaline seawater with $\mathrm{pH}$ ranging from 1 to 13 was utilized to test the separation ability of the NPM under harsh environment. For emulsified oil/water filtration process, adhesion of the oil phase on the membrane surface is the main reason for deterioration of wetting selectivity. Therefore, low adhesion of oil on the membrane is an important factor for long-term oil-in-water emulsion separation with high selectivity and efficiency. Therefore, continuous and cyclic separations were carried out to test the ability of the membrane to resist foulants such as surfactant, BSA and HA. Last, the mechanism of the superwettability of the NPM was explained. 


\subsection{Separation Efficiency of Sundry Organic Solvent/water}

\section{Emulsion}

The separation efficiencies of the as-described NPM along oil/water separation process were testified via a series of experiments presented in Figure 5-1. To demonstrate the separation efficiency of the membrane, six types of insoluble organic solvents were chosen considering their massive use in manufacturing, including petroleum ether, hexane, chloroform, dichloromethane, toluene and carbon disulfide. A surfactant-free oil/water mixture was prepared by sonicating a $10 \%$ petroleum ether-in-water mixture for 10 minutes in water bath. As shown in Figure 5-1a, NPM5 with a diameter of $8 \mathrm{~mm}$ and thickness of $200 \mu \mathrm{m}$ was fixated in between a funnel and an Erlenmeyer flask. The oil-in-water emulsion was transferred into the funnel, which was in direct contact with NPM5. As can be seen from Supplementary Movie 2, water instantaneously soaked into the membrane. The oil-water emulsion was demulsified once in touch with the membrane with water permeated through NPM5 and oil remained above. The filtered water was examined (Figure 5-1d) to study the separation efficiency by counting the oil droplet residue after separation. Observing from the optical microscopy collected from the filtrate, no visible droplet was observed in images obtained from the same batch (Figure 5-1e). The diameter distribution of the oil droplets before separation demonstrates that the diameter of oil droplets is most likely to fall into a range of 5-20 $\mu \mathrm{m}$ (Figure 5-1g). The flux reduced as the thickness of the membrane increased. The flux can reach $12740 \mathrm{~L} \mathrm{~m}^{-2} \mathrm{~h}^{-1}$ when testing a single layer of the NPM, while decreasing to $3981 \mathrm{~L} \mathrm{~m}^{-2} \mathrm{~h}^{-1}$ with a tri-layered membrane (Figure 5-lf). In addition, the separation efficiency and consistency were measured to assess the oil/water separation performance. Similar phenomenon was observed among other organic solvents/water 
mixture. After organic solvent/water mixtures were poured into the funnel, water quickly permeated through the membrane, whilst the organic solvents remained above the membrane, showing excellent separation capability of the material among a wide range of immiscible solutions. 

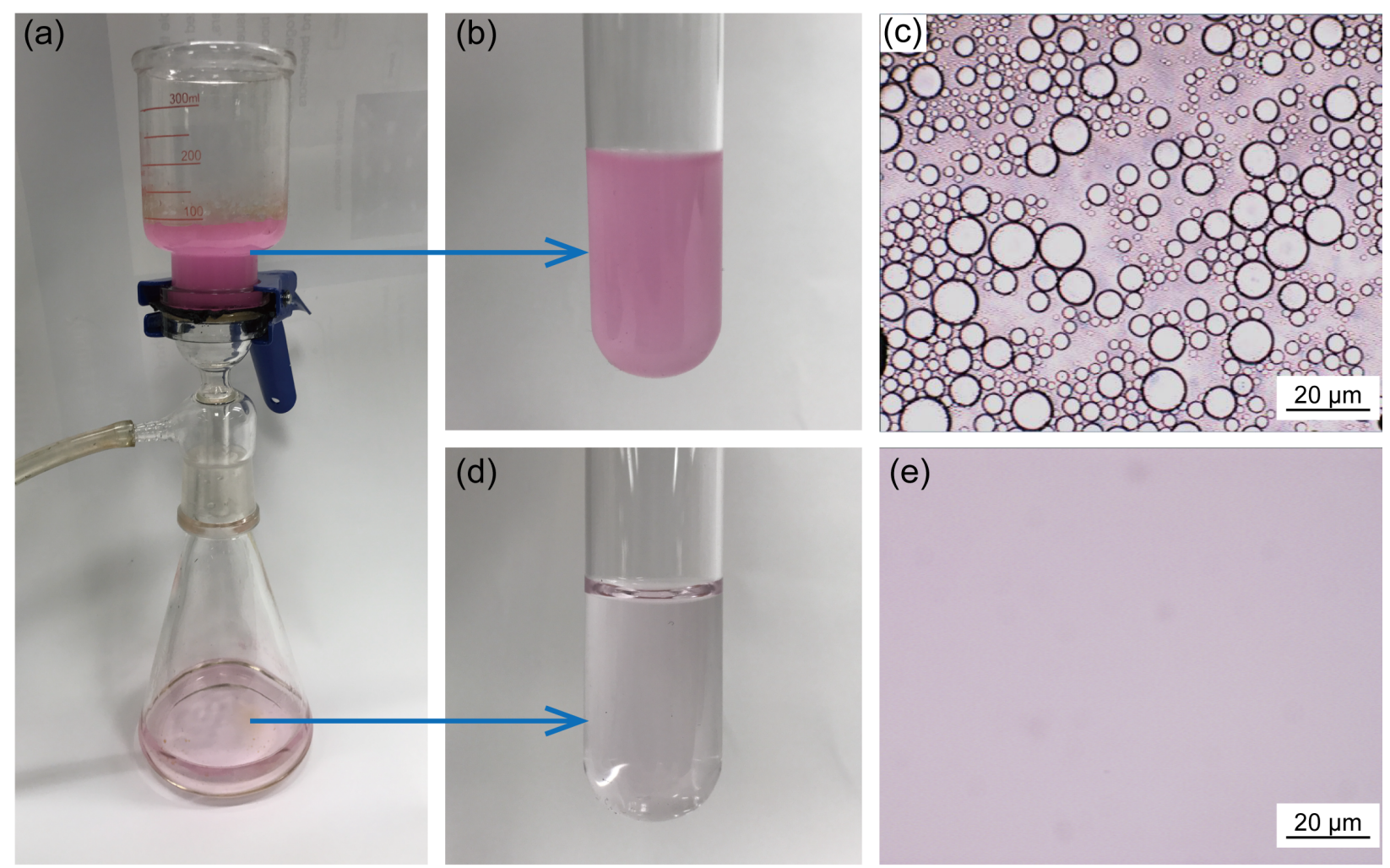

(f)

(g)
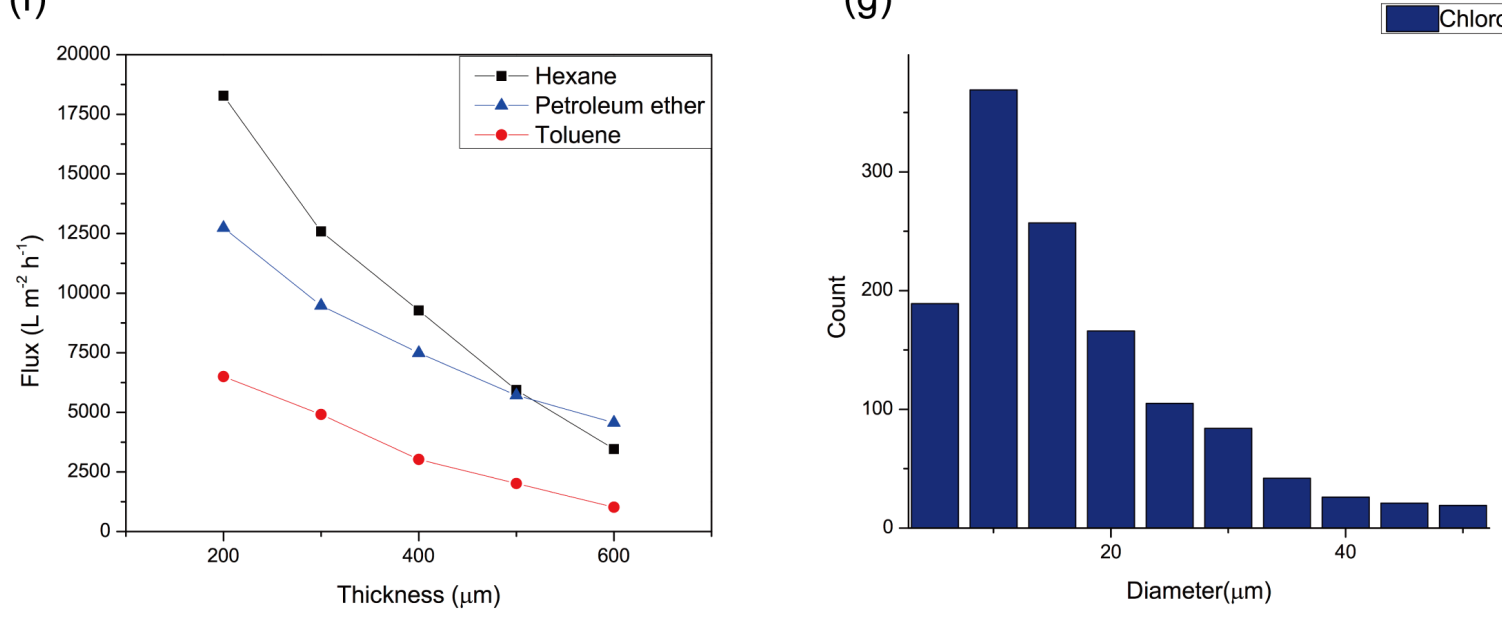

Figure 5-1. Oil/sea water emulsion separation installation with optical microscopy of emulsions before and after separation, together with the separation flux and Particle size distribution of the petroleum ether-in-water mixture. 


\subsection{Membrane Permeability}

Membrane permeability was comprehensively investigated. The fluxes of sundry solvent-inwater emulsions were measured as shown in

Figure 5-2a. As oceanic crude oil spill is one of the most encountered scenarios in industrial oil/water separation, artificial sea water was prepared to simulate real-life separation condition (Table 5-1). Among all the organic solvents and oils used, the separation fluxes are all above $2638 \mathrm{~L} \mathrm{~m}^{-2} \mathrm{~h}^{-1}$ for surfactant free emulsion and $1271 \mathrm{~L} \mathrm{~m}^{-2} \mathrm{~h}^{-1}$ for surfactant-stabilized emulsion. The flux of hexane-in-water emulsion is the highest $\left(12689 \mathrm{~L} \mathrm{~m}^{-2} \mathrm{~h}^{-1}\right)$, while the flux of surfactant-stabilized hexane-in-water emulsion is $3962 \mathrm{~L} \mathrm{~m}^{-2} \mathrm{~h}^{-1}$. There is a slight higher difference of fluxes between surfactant-free and surfactant-stabilized emulsion in hexane than other organic solvent. This phenomenon is possibly due to higher impact of SDS (surfactant used in this thesis) on emulsification of hexane compared to the impact of SDS on the dispersion of other non-soluble organic solvents in water, which further result in smaller hexane droplets in the surfactant-stabilized emulsion. With smaller oil droplets, the emulsion is harder to be demulsified at the surface of NPM, hence the lower flux and more significant difference between the surfactant-free and surfactant-stabilized emulsion. To further confirm this assumption, dynamic light scattering (DLS) were carried out to test the droplet size of hexane and other organic solvents-in-water surfactant-stabilized emulsion. Here, we will compare hexane and petroleum ether as an example. As can be seen from the DLS results in 
Figure 5-3a-d, the average droplet size of surfactant free hexane-in-water emulsion is $2327 \mathrm{~nm}$ while the average droplet size of surfactant stabilized hexane-in-water emulsion is $510.4 \mathrm{~nm}$, which is a $78 \%$ reduction in hexane droplet size with the presence of surfactant. Meanwhile, the average droplet size of surfactant free petroleum ether-in-water emulsion is $1873 \mathrm{~nm}$ while the average droplet size of surfactant stabilized petroleum ether-in-water emulsion is $713.4 \mathrm{~nm}$, which is $62 \%$ reduction in petroleum ether droplet size with the presence of surfactant. The moderately larger change of hexane micelle size after inducing surfactant is coherent with the bigger difference of fluxes of separating surfactant-free and surfactant-stabilized emulsions. In addition, when comparing surfactant free hexane-in-water emulsion and petroleum ether-inwater emulsion, the larger droplet size of hexane is consistent with the result of slightly higher flux during the separation compared to petroleum ether. 


\begin{tabular}{|l|l|}
\hline Compound & Mass $(\mathrm{g})$ \\
\hline $\mathrm{NaCl}$ & 23.98 \\
\hline $\mathrm{MgCl}_{2}$ & 5.03 \\
\hline $\mathrm{Na}_{2} \mathrm{SO}_{4}$ & 4.01 \\
\hline $\mathrm{CaCl}_{2}$ & 1.14 \\
\hline $\mathrm{KCl}$ & 0.70 \\
\hline $\mathrm{NaHCO}$ & 0.17 \\
\hline $\mathrm{KBr}$ & 0.10 \\
\hline $\mathrm{H}_{3} \mathrm{BO}_{4}$ & 0.025 \\
\hline
\end{tabular}

Table 5-1. The composition of artificial seawater (The total mass of the compound and the seawater is $1 \mathrm{~kg})$. 
(a)

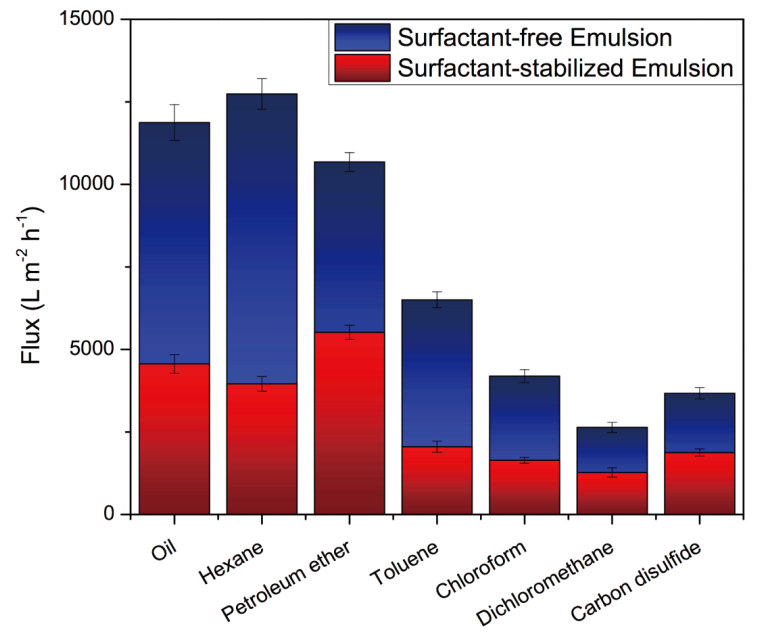

(c)

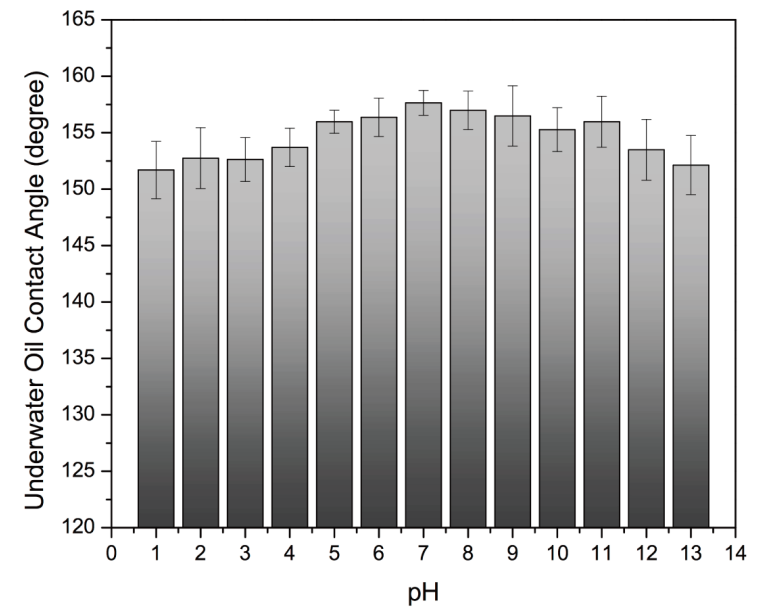

(b)

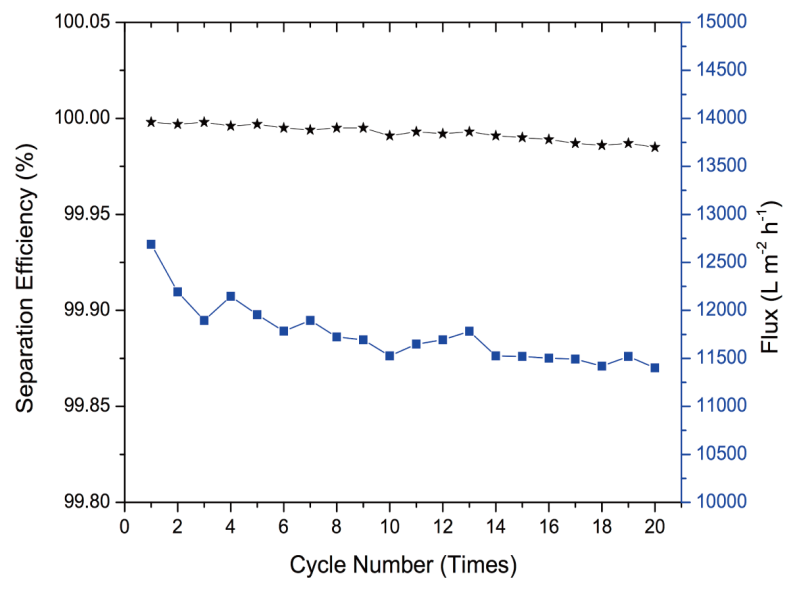

(d)

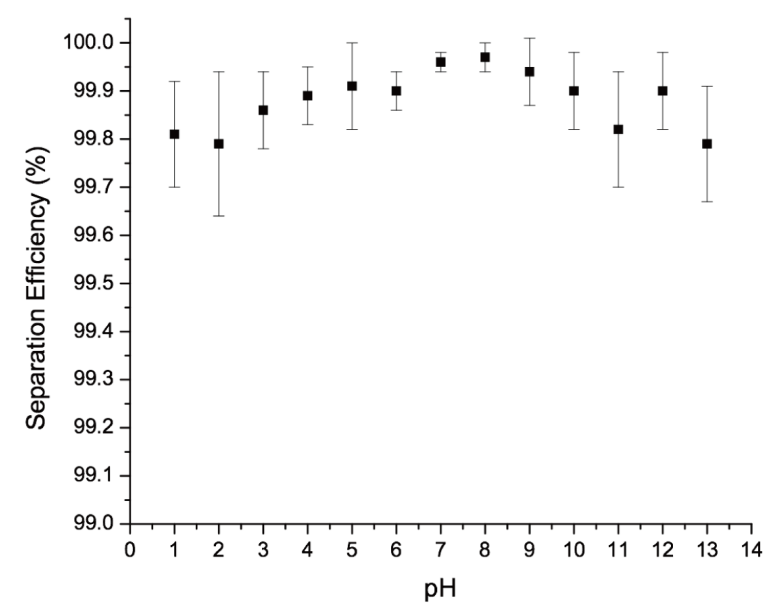

Figure 5-2. The separation flux of a sequence of surfactant-free and surfactant-stabilized oil-inseawater emulsions penetrating through the membrane. 
To characterize the consistency of the separation performance, repeated separation was carried out. As a result, the membrane exhibited high separation efficiency $(>99.99 \%)$ in oil-in-water emulsions after 20 times of separation, indicating the stability of the membrane (

Figure 5-3b). Furthermore, the $0.01 \%$ SDS stabilized emulsions are left unshaken for a prolonged period of time. As can be seen from

Figure 5-3e-f, no stratification was observed after 15 days, exhibiting excellent separation efficiency of stabilized emulsions of the membrane. According to the above separation results, the NPM shows prominent performance in oil or organic solvents/water separation for surfactant-free or surfactant-stabilized oil-in-water emulsion. The membrane can quickly absorb water as soon as in contact with the emulsion mixture. Afterwards, the superhydrophilic and underwater superoleophobic properties allow water to instantly permeate through the membrane with the oil phase remaining above. 
(a)

Size Distribution by Intensity

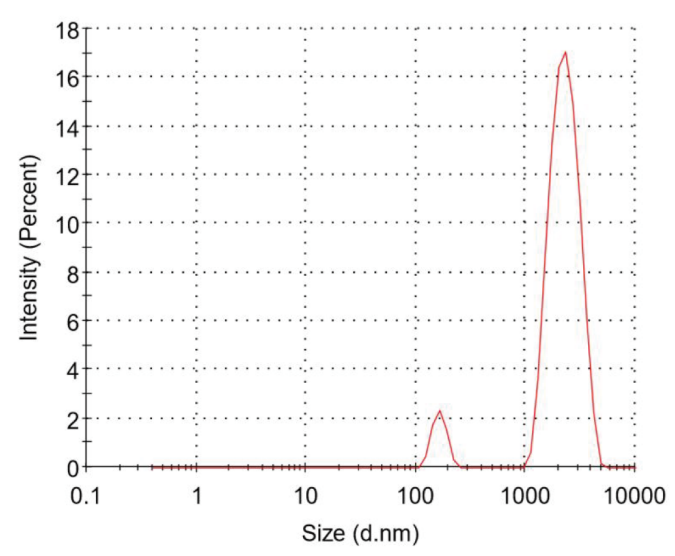

(c)
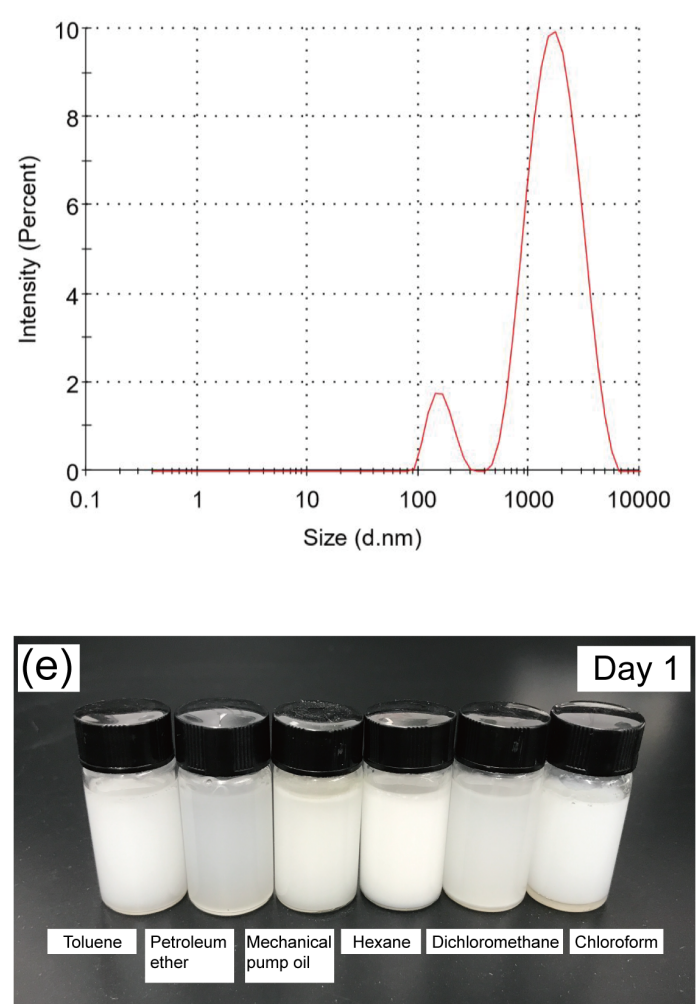

(b)

Size Distribution by Intensity

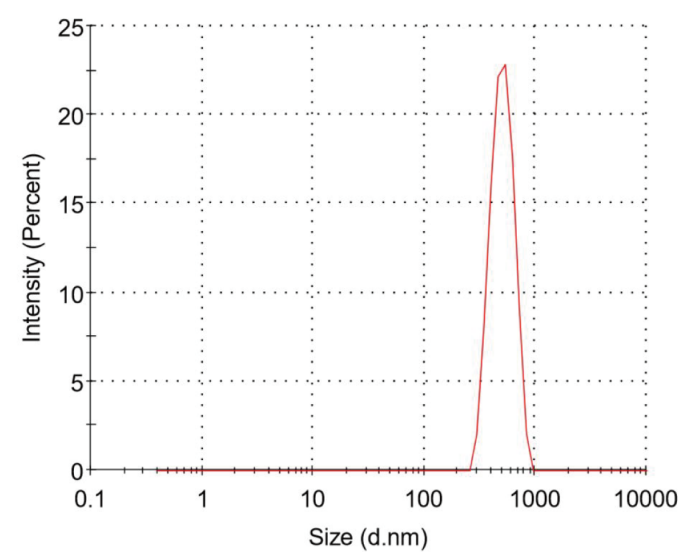

(d)

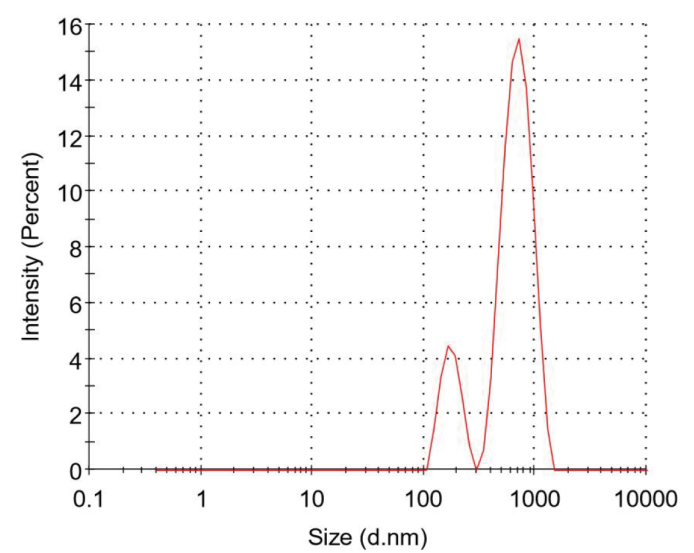

Figure 5-3. Distribution of oil drops with different diameter ranges with and without emulsifier stabilization and images of the surfactant stabilized 10\% oil-in-water emulsions on day 1 and day 15. 


\subsection{Emulsion Separation Under Harsh Environment}

Another feature of this work is to show the excellent functionality under harsh conditions. The NPM is capable of resisting acidic/alkaline condition under severe $\mathrm{pH}$ environment.

Figure 5-2c shows water contact angles of NPM in artificial sea water with various $\mathrm{pH}$. When the membrane was immersed in acid/alkaline seawater with $\mathrm{pH}$ ranging from 1 to 13 , the oil droplets exhibit spherical shape and all the underwater contact angles are above $151^{\circ}$, showing consistent superoleophobic characteristic. The oil-in-water acidic or alkaline emulsion was prepared by mixing $10 \%$ of organic solvent and artificial seawater with the addition of $\mathrm{HCl}$ or $\mathrm{NaOH}$ solution to adjust the $\mathrm{pH}$. The filtration device was the same as in the previous one, with the membrane fixated in between the rims of flask and the funnel. After the equipment was set up, the emulsion was poured into the funnel under 0.2 bar pressure. In this case, hexane-in-water emulsion was selected as an example. During the separation, the cloudy emulsion transformed into transparent, clear water in the flask, indicting excellent separation result. The as-prepared NPM can separate stabilized micro and nanoscale oil-in-water emulsion effectively under acid, basic and briny conditions. Owing to the chemical-resistance nature of PVA, the described membrane can retain the superhydrophilicity/underwater superoleophobicity property under harsh environment. To gauge the separation efficiency under severe environment, the water purity after separation was measured as can be seen from

Figure 5-2d. The membrane shows extremely high efficiency (>99.6\%) under a wide range of $\mathrm{pH}$ value, indicating its stability under complex separation environment. The above results suggest that the NPM has the capability of resisting acidic/basic environment under a series of $\mathrm{pH}$ conditions. 


\subsection{Anti-fouling Capability of the NPM}

Filtration efficiency in emulsion separation is often impeded by the fouling of oil on the membrane surface or clogging in pores. The oil/organic solvent residue adheres to the membrane and weaken the superoleophobicity of the membrane, affecting the separation efficiency as well as permeation flux. Therefore, low adhesion between oil and the membrane is an important characteristic to remediate oil fouling and ensure highly efficient oil/water emulsion separation. $[224,232,233]$ Surface dynamic adhesion test was carried out to assess the adhesion behavior between oil droplets and the NPM underwater. As can be seen from Supplementary Movie 1, a dichloromethane droplet was pressed onto a NPM5 and lifted up. During the pressing process, the droplet was deformed into an ellipsoid while during the lifting process, the droplet returned its shape to a sphere and no further deformation was observed when the droplet was detached from the membrane surface. similar phenomenon was observed when highly viscous mechanical pump oil was utilized in the test. The results prove the extremely low adhesion between the oil droplet and the NPM, which is beneficial for relieving oil-fouling during separation.

In addition, the NPM5 also possess easy-cleaning property. During the self-cleaning experiment, the NPM1 and NPM5 were immersed in mechanical pump oil, followed by shaking in DI water. As can be seen from Figure 5-4a, oil droplets were easily shed from the NPM5 after shaking in water and the oil droplets can be observed from surface of the water. In contrast, the NPM1 was immersed the mechanical pump oil, and after shaking in water, almost no oil droplet was observed at the top of the water surface, indicating oil fouling on the membrane. The results suggest low adhesion of oil on NPM5 and easy cleaning once fouled with oil. 
To test the long-term anti-fouling property of the NPM, cyclic filtration test was carried out on the NPM with several foulants including surfactant (SDS), BSA and HA. The flux recovery ratio (FRR) was calculated after each cycle of emulsion separation and washing. Mechanical pump oil was utilized in this test considering its similar composition to crude oil (petroleum distillates) and high viscosity. Firstly, surfactant free mechanical pump oil-in-water emulsion was tested and the relative flux result is shown in Figure 5-4b. The FRR of NPM5 maintained beyond 98\% after 420 min of repeated separation and washing process, comparing the FRR of $87 \%$ of NPM1. The relative flux of NPM5 dropped to 0.51 after 6 cycles, while the relative flux of NPM1 dropped to 0.37, demonstrating that the lower hydrophilicity of NPM1 due to the lack of PPy nanoparticles coating results in more severe fouling even without the surfactants or other contaminants. $0.1 \mathrm{mg}$ $\mathrm{ml}^{-1}$ surfactant (SDS) was mixed into the mechanical pump oil and water and as can be seen from Figure 5-4c, the FRR of NPM5 remained $97 \%$ while the FRR of NPM1 dropped to $85 \%$ after 420 min of cyclic separation and washing procedure. The relative flux of NPM1 decreased from 0.47 after first cycle of separation to 0.34 after 6 cycles, suggesting more severe fouling during the surfactant stabilized emulsion separation. The results for another two contaminants, HA and BSA are shown in Figure 5-4d and Figure 5-4e, in which $1 \mathrm{mg} \mathrm{ml}^{-1} \mathrm{HA}$ and $1 \mathrm{mg} \mathrm{ml}^{-1}$ BSA were added to the surfactant stabilized emulsion, respectively. The FRR of NPM 5 maintained at the level of $95 \%$ after cyclic test of both HA mixed emulsion and BSA mixed emulsion. In contract, the FRR of NPM1 decreased to $71 \%$ for HA mixed emulsion and $73 \%$ for BSA mixed emulsion, respectively. The results confirmed the effect of PPy nanoparticles in the fouling-resistant property of the membrane against heavy oil and multiple contaminants. The NPM5 shows outstanding anti-fouling property as well as longevity for heavy-duty emulsion separation. 


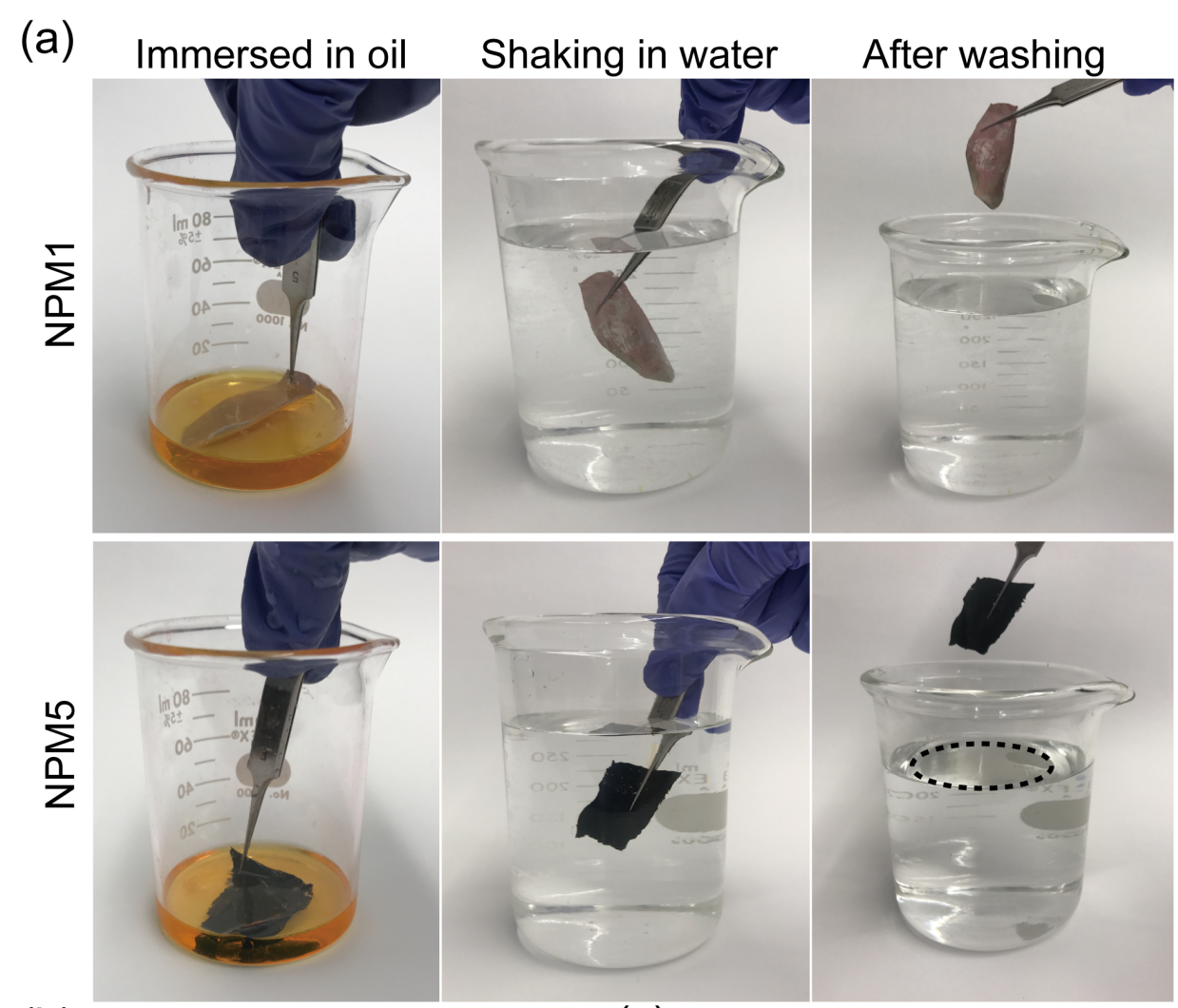

(b)

(c)

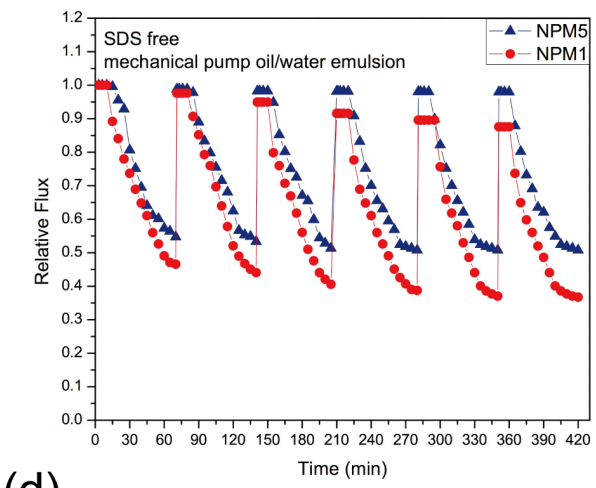

(d)

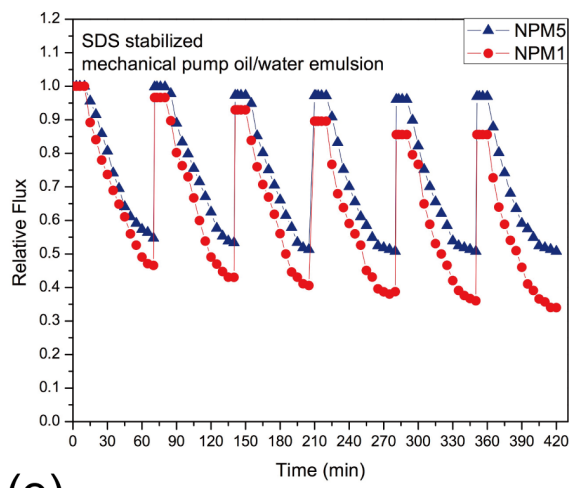

(e)
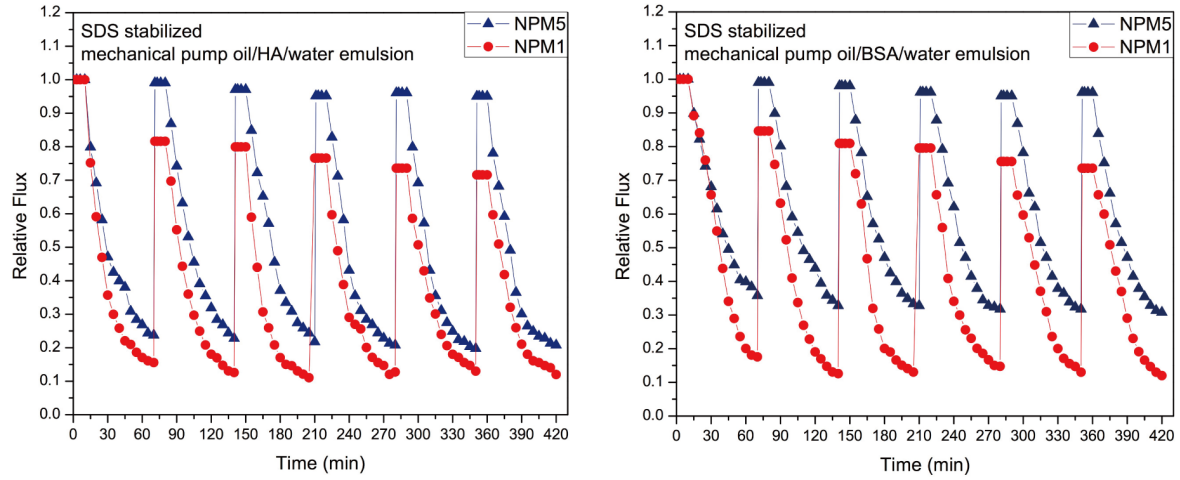

Figure 5-4. Anti-fouling and resilience characteristics of the NPM. 


\subsection{Super Wettability Mechanism}

To thoroughly explain the ultrahigh oil-water separation performance, the superoleophobicity/superhydrophilicity of the NPM was studied. According to Young's equation, the solid surface wetting behavior is generally calculated by the contact angle as follows:

$$
\cos \theta=\frac{\gamma_{s g}-\gamma_{s l}}{\gamma_{l g}}
$$

in which $\gamma_{\mathrm{sg}}$ is the solid-vapor interfacial energy, $\gamma_{\mathrm{sl}}$ is the solid-liquid interfacial energy and $\gamma_{\mathrm{lg}}$ is the liquid-vapor interfacial energy.

Young's equation is originally applied to the oil on the solid interface with the presence of air. However, it is also applicable to the oil droplet on a solid surface in water. Thus, the following equation can be generated

$$
\cos \theta_{3}=\frac{\gamma_{o i l-g} \cos \theta_{1}-\gamma_{\text {water }-g} \cos \theta_{2}}{\gamma_{\text {oil-water }}}
$$

Where $\gamma_{\mathrm{oil}-\mathrm{g}}$ is the oil-gas interface tension, $\theta_{1}$ is the oil contact angle with the presence of air, $\gamma_{\text {water-g }}$ is the water/gas interface tension, $\theta_{2}$ is the water contact angle with the presence of air, $\gamma_{\text {oil-water }}$ is interface tension of oil and water interface, and $\theta_{3}$ is the oil contact angle with the presence of water.

According to Equation 7, the phenomenon of a hydrophilic surface in air becoming oleophobic can be explained. If we use dichloromethane as an example, the water surface tension $\gamma_{\mathrm{water}-\mathrm{g}}$ is $73 \mathrm{mN} \mathrm{m}^{-1}$, while the interfacial tension of gas $\gamma_{\text {oil-g }}$ is $25.6 \mathrm{mN} \mathrm{m}^{-1}$.[234] The dichloromethanewater interfacial tension $\gamma_{\text {oil-water }}$ is $28.3 \mathrm{mN} \mathrm{m}^{-1}$.[235] With the presence of air, the 
dichloromethane contact angle on NPM $\theta_{1}$ measured is almost 0 , while water contact angle $\theta_{2}$ was 57.5. As indicated in Equation $7, \cos \theta_{3}=-0.49$, therefore, $\theta_{3}$ equals to $155^{\circ}$, indicating the NPM behaved as a superoleophobic surface in water. This theoretical speculation aligns with the experimental results, in which the membrane is oleophilic in air while showing superoleophobicity property under water. In a regular water contact angle situation, the material will show a hydrophobic property if only liquid surface tension is larger than 4 times of the solid surface tension. Since typical surface tensions of non-polar organic solvents and oils are generally 20 to $30 \mathrm{mN} \mathrm{m}^{-1}$, the surface tension of a material should be smaller than $10 \mathrm{mN} \mathrm{m}^{-1}$ to make it oleophobic. Nonetheless, the air phase was exchanged by water phase in this study, which implemented oleophobic characteristic on materials with high surface tension. Cassie et al.[85] established a model related to the contact angle in a solid/water/air system. When the air phase was displaced by the water phase, Cassie's model can be written as:

$$
\cos \theta_{3}^{\prime}=f \cos \theta_{3}+f-1
$$

where area fraction of solid is referred as $\mathrm{f}$, contact angle of an oil droplet on a smooth surface in water is referred as $\theta_{3}$, and the contact angle of an oil droplet on a rough surface in water is referred as $\theta_{3}^{\prime}$. The rougher the solid phase is, the lower is the area fraction. For a nanostructured surface as NPM, $\mathrm{f}$ is close to 0 . Thus, $\theta_{3}^{\prime}$ is close to $180^{\circ}$ according Equation 8 . In this study, the PPy particles coated on the electrospun PVA nanofibers, resulting in lower area fraction of the solid substrate. Thus, the PPy coated PVA membrane(NPM5) shows higher superoleophobicity than PVA membranes prior to PPy coating (NPM1). This assumption is validated with the experiment result, where the underwater contact angle of PVA membrane increased from $123^{\circ}$ to $158^{\circ}$ with coated PPy nanoparticles. 


\section{Chapter 6 Solar Vapor Generation}

\subsection{Introduction}

In this chapter, NPM as a solar vapor generator is discussed regarding the effect of PPy on the solar vapor efficiency, evaporation rate and desalination performance. The densely packed PPy nanoparticles on the PVA fibers is serving the purpose of quickly harvesting the solar light and converting it into thermal energy. The converted solar energy can be directly delivered to the small amount of water in the molecular meshes. Compared to other research using PPy as light absorber, the PPy in this work is an elastic network formed by slow oxidation process, which made the PPy coverage on the nanofibers surface homogeneous and continuous. Lastly, on-site desalination testing of the NPM was carried out to assess small scale application of NPM.

\subsection{The Solar Steam Installation Under Various Illumination}

\section{Concentration}

The hierarchical nano-structure membrane possesses many special characteristics to realize high energy efficiency seawater desalination. The broad band absorption of light and high energy conversion contributes to the localized water heating and high energy efficiency. In water distillation, localized heating is considered as an important quality as the traditional bulk heating of water lead to unnecessary energy loss to the unevaporated bulk portion of the water[236]. The experiment installation of solar vapor generation is shown in

Figure 6-1a. The installation is composed of water supply, sunlight simulator and a NPM as the functional light converter. The membrane is floating on the surface of a container filled with 
water, while the sun simulator is perpendicularly illuminating the whole system from above. Under solar irradiance, the water molecules at the interface of membrane and air will escape the pores and channels on the membrane and later condense in the chamber as distilled water. The gaps and pores between different layers of the PVA nanofibers (typically $400 \mathrm{~nm}$ in diameter) give rise to channels for sufficient water supply. Considering the characteristics of NPM including the broadband light absorption, low thermal conductivity and high light-to-heat energy conversion, such material is exceptionally suitable for the solar desalination process. NPM5 samples with a diameter $10 \mathrm{~mm}$ and a thickness of $200 \mu \mathrm{m}$ are utilized for solar steam generation.

Figure $6-1 \mathrm{~b}$ shows the temperature change with respect to time under 1 sun irradiation. With ambient temperature of $22{ }^{\circ} \mathrm{C}$, bulk water started with a temperature of $18.9^{\circ} \mathrm{C}$ and reached 27.3 ${ }^{\circ} \mathrm{C}$ after 5 min of illumination. On the contrary, with the presence of NPM5, the temperature of water rapidly increased to $39.4{ }^{\circ} \mathrm{C}$ after 5 min of irradiation, demonstrating a remarkable solarto-heat conversion ability. It is noticeable that with the presence of NPM5 in the water, the temperature reached equilibrium after $10 \mathrm{~min}$ of sun illumination, demonstrating the rapid response time for this water distillation system.

Figure 6-1c represents the effect of PPy on mass change under 1 sunlight and 0.5 sun illumination with respect to time. Once the solar steam reaches an equilibrium state, the evaporation rates under various Copt were recorded in

Figure $6-l$ d. As can be observed in

Figure 6- $l \mathrm{~d}$, solar vapor generation with the NPM is more substantial than without NPM present in the bulk water under all solar concentration conditions. In particular, the evaporation rate with 
the NPM structure for Copt $=1$ reaches $\sim 2.87 \mathrm{~kg} \mathrm{~m}-2 \mathrm{~h}-1$, which is almost 7 times higher than the evaporation rate of pure water.

Figure 6-1e represents the experimental thermal efficiency of the solar vapor system. The efficiency of solar vapor generation of the NPM5 reaches up to $76.1 \%$ under $0.8 \mathrm{~kW} \mathrm{~m}^{-2}$ solar flux, $87.5 \%$ under $1 \mathrm{~kW} \mathrm{~m}^{-2}$ solar flux, contrasting $22.7 \%$ of pure water. The equilibrium evaporation rates and energy efficiencies of NPM1, NPM2, NPM3, NPM4 and NPM5 under 1 sun irradiation were summarized in

Figure 6-1f. As can be observed, the evaporation rate of NPM increase along with the reaction time of PPy polymerization. Accordingly, the equilibrium evaporation rates of NPM1, NPM2, NPM3, NPM4 and NPM5 are 1.568, 2.257, 2.464, 2.71 and $2.872 \mathrm{~kg} \mathrm{~m}^{-2} \mathrm{~h}^{-1}$, and the energy efficiencies are $52.21 \%, 73.37 \%, 79.09 \%, 85.1 \%$ and $87.5 \%$, respectively.

\subsection{Effect of PPy on Solar Vapor Efficiency}

To understand why NPM5 shows such outstanding solar vapor efficiency, the optical properties of NPM1 and NPM5 were measured via a UV-vis Spectrometer Ultraspec 4300 pro from 250$1100 \mathrm{~nm}$. As can be seen from Figure 6-2a, the electrospun PVA membrane (NPM1) shows mediocre light absorption property due to the intrinsically high reflectivity of PVA nanofibers. After the PVA nanofibers are deposited with a layer of closely packed PPy nanoparticles, the surface becomes less reflective (the sunlight cannot be reflected, thus it shows a dark black appearance in macro scale) because of the excellent light absorbability of the PPy. The light absorption of the NPM5 can reach 99.9\% within a broad wavelength range from $250 \mathrm{~nm}$ to 1100 $\mathrm{nm}$. While the absorption of PVA membrane can only reach $65 \%$ at the wavelength of $250 \mathrm{~nm}$ 
and $90 \%$ absorption along most of the wavelength.

The effect of the PPy nanoparticles on the swelling ratios of the NPM is shown in Figure 6-2b. It can be observed that an increase in the deposition of PPy nanoparticles on the PVA fibers yields a slight increase in the swelling ratio of the membrane. The increase of swelling ratio can be explained that the presence of PPy increases the hydrophilicity of the membrane, which results in slightly higher equilibrium swelling ratio.

To systematically evaluate the energy efficiency of the NPM solar vapor devise, the corresponding energy efficiency $(\eta)$ for solar steam generation was calculated using the following formula:

$$
\eta=\dot{m} h_{v} / C_{o p t} P_{0}
$$

where $\dot{\mathrm{m}}$ refers to the mass flux, $\mathrm{h}_{\mathrm{V}}$ refers the evaporation enthalpy of water stored in the membrane, $\mathrm{P}_{0}$ refers to the solar radiation power of 1 sun $\left(1 \mathrm{~kW} \mathrm{~m}^{-2}\right)$, while $\mathrm{C}_{\mathrm{opt}}$ is the optical concentration of the sunlight simulator. It is observed that the evaporation enthalpy of bulk water is larger than that in the NPM (Figure 6-3). Here, water cluster theory can be used to understand this increase in evaporation enthalpy. Either as one molecule or clusters with multiple molecules, water can evaporate and escape the liquid-air interface. Compared to single water molecule, a cluster of water molecules trapped in a porous structure is more likely to escape the matrix. Therefore, with the NPM, the water is more likely to be evaporated from the porous matrix as a result of lower enthalpy.

Figure 6-2c is the IR images of heat distribution of the NPM floating at the air-liquid interface while sunlight irradiation starts from $0 \mathrm{~s}$ to $300 \mathrm{~s}$. Starting from an $18.9^{\circ} \mathrm{C}$ water temperature, the surface temperature of the NPM rapidly increases to $41.6{ }^{\circ} \mathrm{C}$ during the first $300 \mathrm{~s}$ of irradiation. The thermal distribution of the membrane surface shows minor changes after $300 \mathrm{~s}$, 
ultimately reached the highest at $42.3{ }^{\circ} \mathrm{C}$, comparing to the water temperature underneath the membrane shows insignificant rise owing to the low thermal conductivity of the material. These data suggest that the membrane has a highly localized heating property which is beneficial to energy efficiency and steam generation. 
(a)

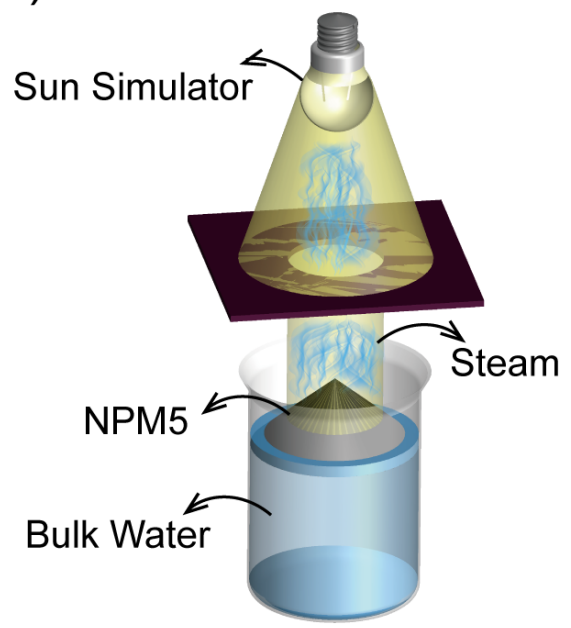

(c)

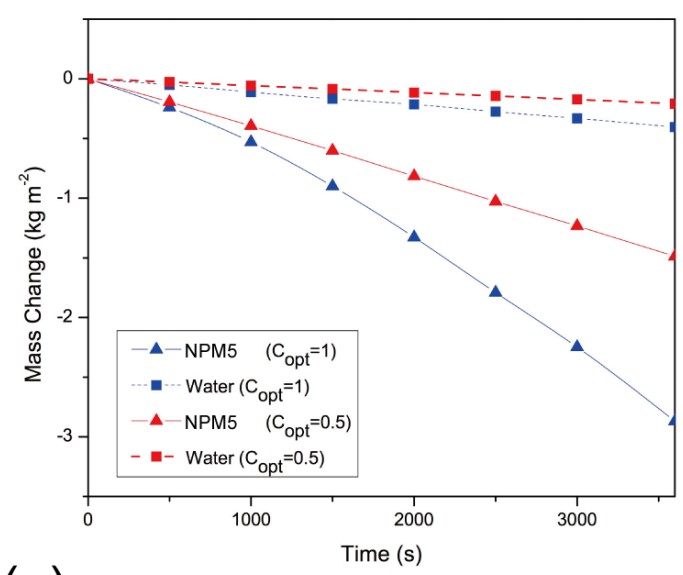

(e)

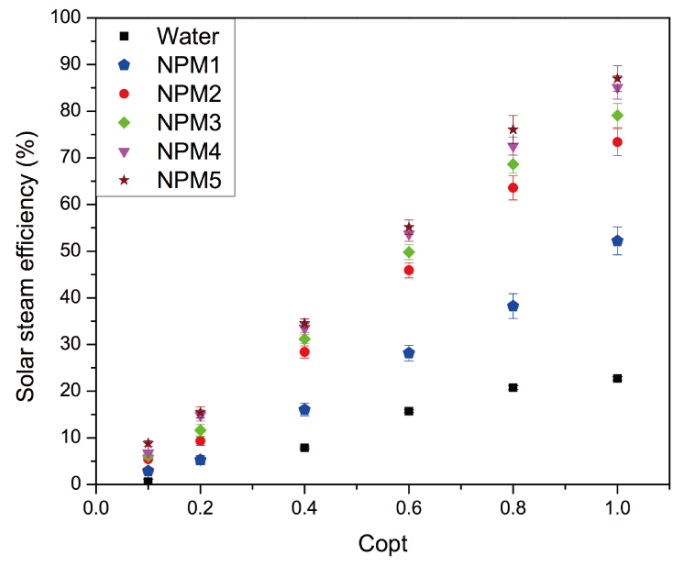

(b)

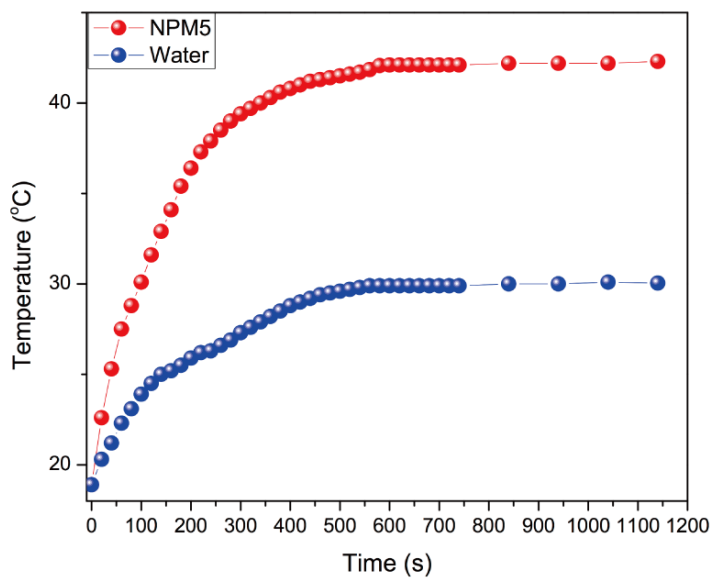

(d)

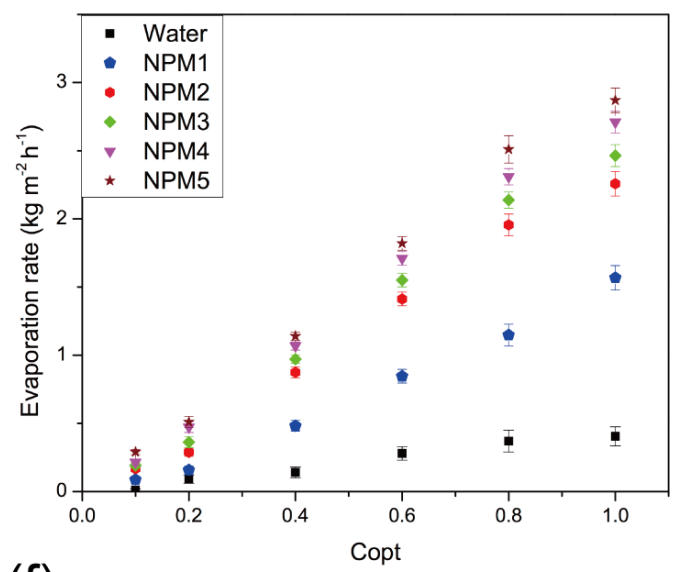

(f)

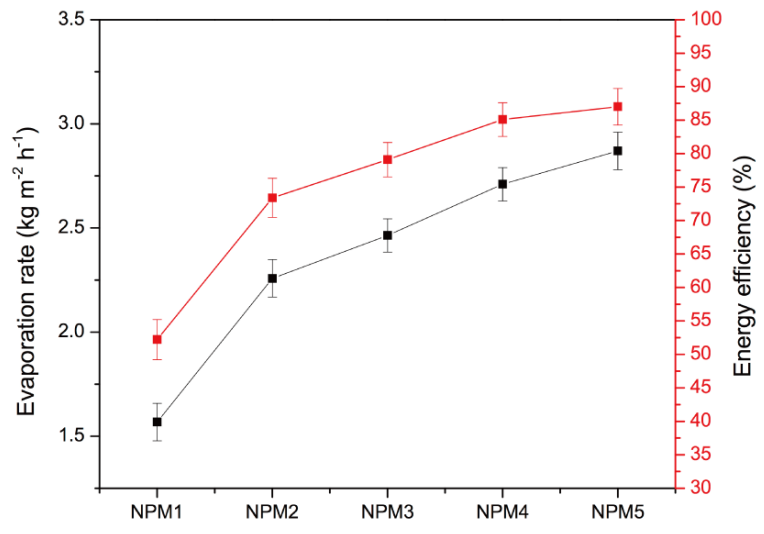


Figure 6-1. Schematic set up for solar-vapor desalination unit used in the experiment and the summary of energy efficiencies and evaporation rates of NPM with various degree of PPy coating.

(a)

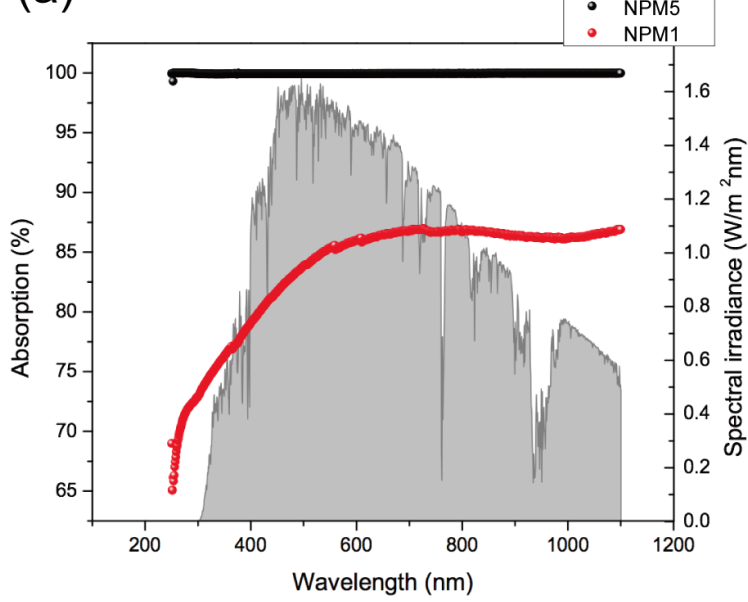

(c)

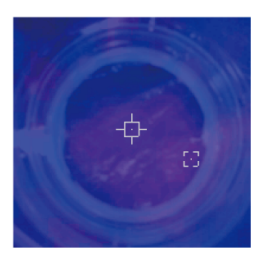

$0 \mathrm{~s}$

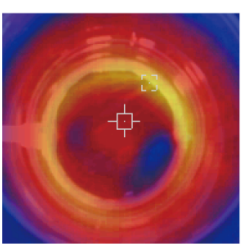

$60 \mathrm{~s}$

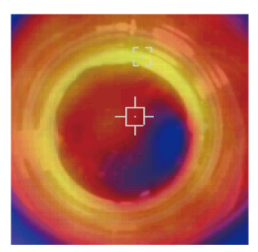

$120 \mathrm{~s}$ (b)

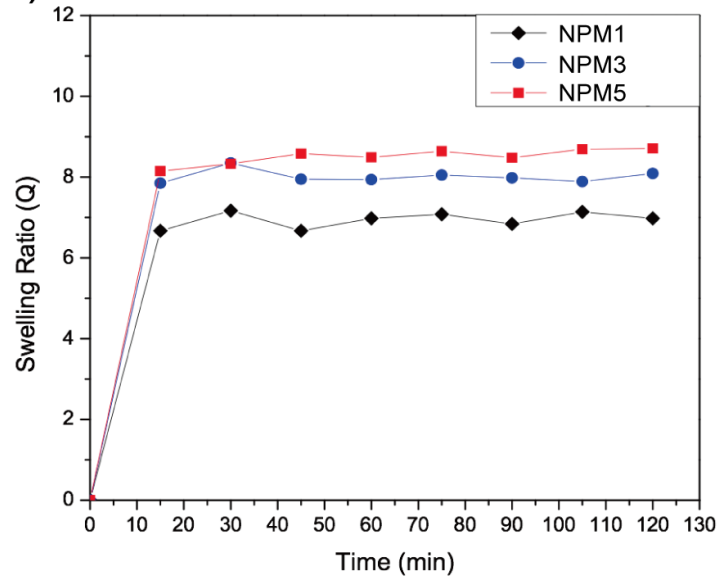

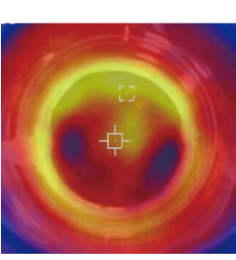

$180 \mathrm{~s}$

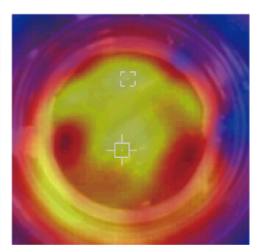

$240 \mathrm{~s}$

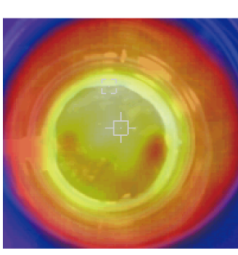

$300 \mathrm{~s}$

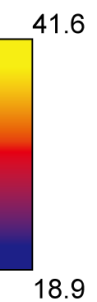

Figure 6-2. UV-vis NIR spectra comparison of NPM, swelling ratios of NPM1, NPM3 and NPM5 and IR images of the thermal dispersion of the NPM5. 


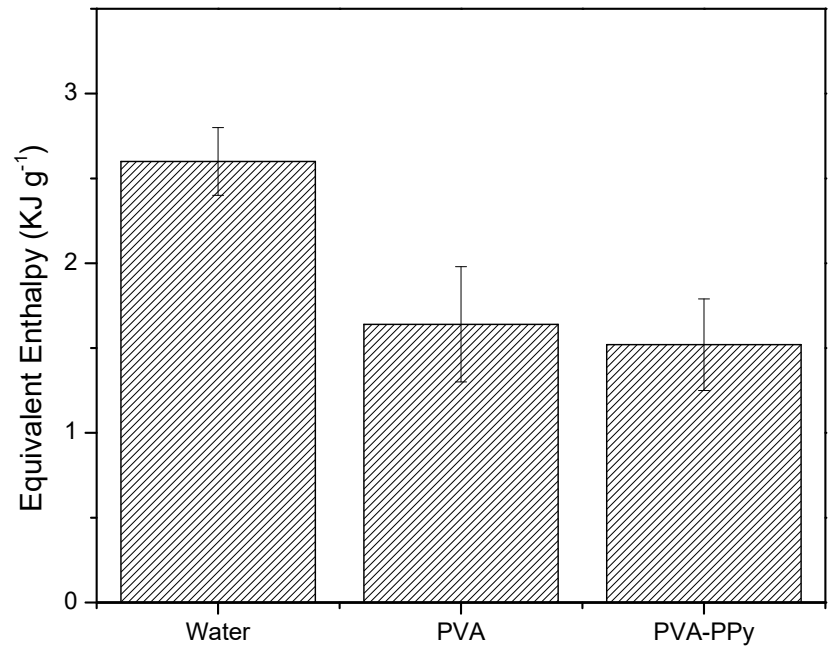

Figure 6-3. The calculated equivalent enthalpy of bulk water, electrospun PVA membrane and PVA-PPy membrane based on the evaporation rates in dark condition. 


\subsection{Desalination Performance}

To quantitatively gauge the distilled water quality, four artificial seawater of various salinities, were used and carefully evaluated by conductivity tests, the Baltic sea $(0.8 \mathrm{wt} \%)$, the average salinity of the world ocean (3.5 wt\%), the Red Sea (4 wt\%) and the Dead Sea (10 wt $\%)$, respectively[237]. It is obvious that the $\mathrm{Na}^{+}$concentrations in the collected desalinized water are all distinctively decreased compared with the ion concentrations prior to the desalination. The salinity results of the four samples are all below the WHO and Health Canada drinking water standards (Figure 6-4a). Moreover, a real sea water sample (from North Pacific Ocean) was utilized for on-site desalination test with NPM5. The concentration of $\mathrm{Na}, \mathrm{Ca}, \mathrm{Mg}$ and $\mathrm{K}$ ions are monitored to measure the desalination efficiency. From Figure 6-4b, it is observed that the ion concentrations after desalination are 2 orders of magnitude below the original ion concentrations in seawater among all four samples. Durability is another feature of the NPM, which enables a durable and consistent desalination performance. Figure 6-4c illustrates the evaporation efficiency of a NPM under cyclic test of 20 times. The test was held under 1 sun irradiance and the membrane was washed and dried after each use. The evaporation rates of the 20 cycles fit in a narrow range between 2.72 and $2.91 \mathrm{~kg} \mathrm{~m}^{-2} \mathrm{~h}^{-1}$. The stability of solar vapor generation performance was also tested under acidic/base harsh environment, where seawater was treated to achieve specific $\mathrm{pH}$ (ranging from 1 to 13). As shown from Figure 6-4d, the evaporation rates of

NPM5 are all above $2.74 \mathrm{~L} \mathrm{~m}^{-2} \mathrm{~h}^{-1}$ with the energy efficiencies all above $83.02 \%$, presenting excellent solar vapor generation ability over a wide range of $\mathrm{pH}$ conditions. 
(a)

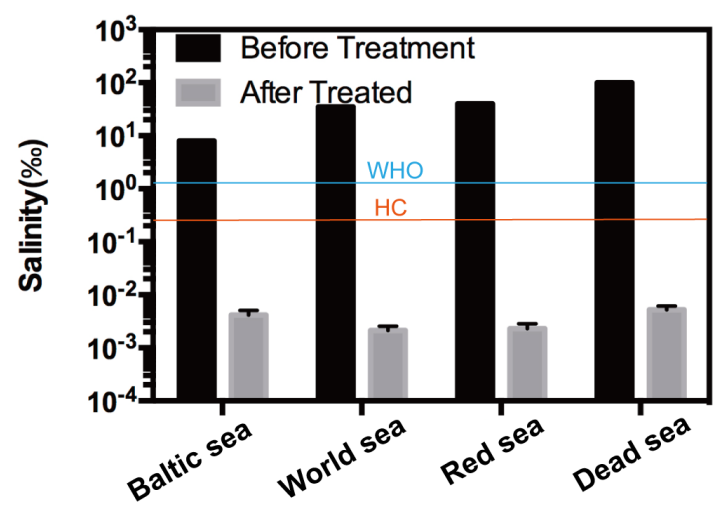

(c)

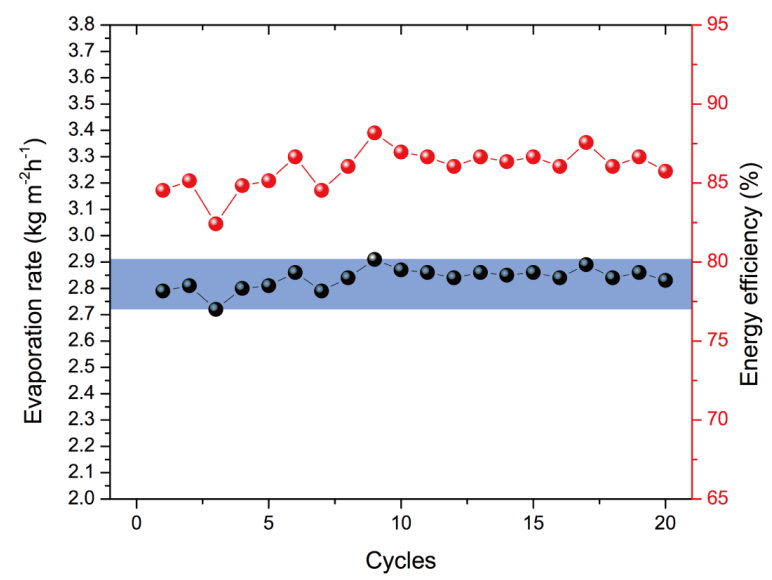

(b)

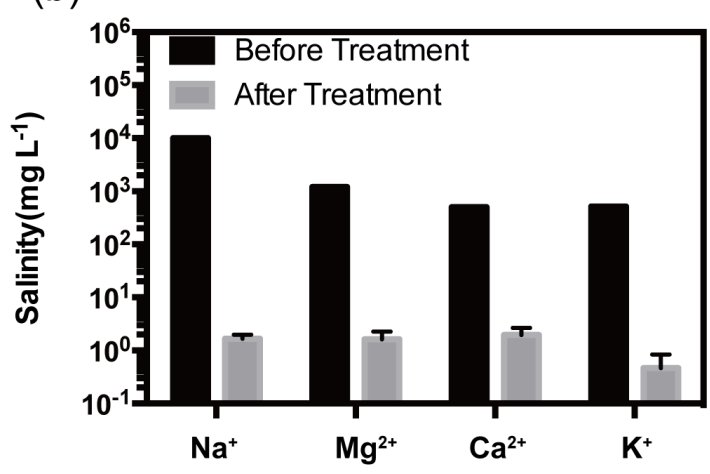

(d)

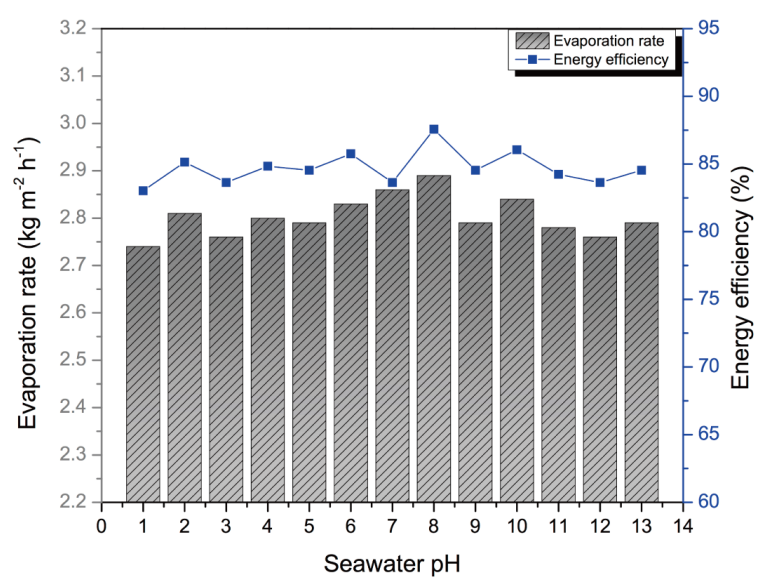

Figure 6-4. The salinity test result using NPM5 with salinity result before desalination in comparison to after desalination and the evaporation rate and energy efficiencies of NPM5 under various seawater conditions with 1 sun illumination. 


\subsection{On-site Solar Vapor Generation Performance}

A prototype of water purification system utilizing NPM5 was carried outdoor for on-site purification testing. Here, an NPM5 was fabricated in the dimension of $10 \mathrm{~cm}$ in diameter and $200 \mu \mathrm{m}$ in thickness (Figure 6-5e). the brine tank with a floating NPM5 at the surface was placed in a plastic chamber for steam condensation. The purified water was delivered by a tube connecting condensation chamber and a water bottle. To testify the desalination function of the water purification system, the system was then exposed to sunlight. The natural sunlight led to an average all-day purified water yield of $\sim 1.2 \mathrm{~L} \mathrm{~m}^{-2} \mathrm{~h}^{-1}$. The temperature of seawater in the flask remained around $30{ }^{\circ} \mathrm{C}$ under sun exposure, despite that the temperature inside of the condenser climbed up to $42{ }^{\circ} \mathrm{C}$, demonstrating a prominent heating localization effect of the NPM. In addition, the fact that the system is still collecting purified water during cloudy days proved that the NPM supported solar vapor generation under periods of various solar exposure, revealing its significant potential for industrial applications.

Additionally, to demonstrate a thermal distribution of the solar vapor system, a COMSOL Multiphysics (a simulation software for multiphysics simulation) was constructed to simulate the heat loss of the system (Figure 6-5c, d). A steady-state heat transfer module was used because the actual experiment occurred under quasi-steady conditions, with constant evaporation rate. Here, some boundary conditions and parameters were used in the COMSOL model are listed below.

The results of the COMSOL model are shown in Figure 6-5c,d. With these boundary and parameters matched, the temperature mapping in the COMSOL model matches the experimental results, and the fitted evaporation rate are 2D direct contact (53\%) and cone-shape transpiration device $(83 \%)$ respectively. The radiation losses, convection losses and conduction loss of $2 \mathrm{D}$ 
direct contact have account for $\sim 7 \%$ and $\sim 30 \%$. 3D artificial transpiration device have account for $\sim 5 \%$ and $\sim 1 \%$. These data are consistent with the results of experiment. 


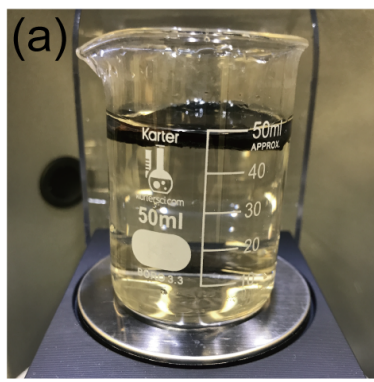

(c)

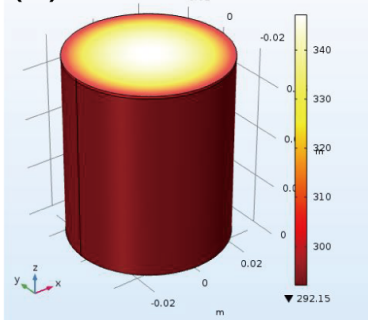

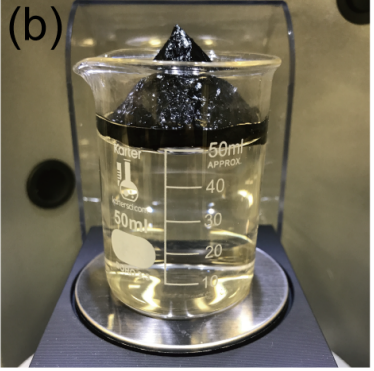

(d)

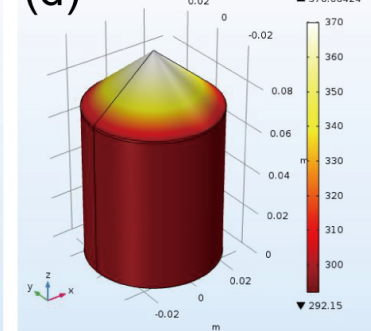

(e)

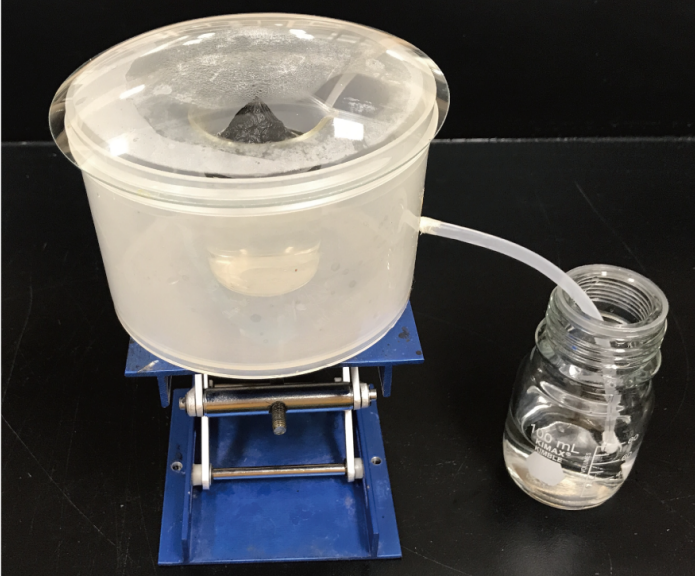

Figure 6-5. COMSOL model of calculated device with $2 D$ indirect contact and $3 D$ artificial transpiration. 


\section{Chapter 7 Conclusion and Future Works}

We have successfully established a highly effective water purification system with facile synthesis of NPM as light absorber and oil/water separator. Instead of representing oil/water separation or solar steam generation as the single feature of the purification system, for the first time we combined these two elements together and created a dual-functional purification system. The deposition of PPy nanoparticles onto PVA nanofiber enbales NPM5 with superhydrophilicity which facilitates rapid water permeation. Making most use of the superhydrophic/superoleophobic hierachical nano-structure and ultra-high light absorption of NPM5, the system is capable of fisrtly, separate the mixed oil and water and secondly, generate solar steam for water desalination. The oil/water separation efficiency is over $99.99 \%$ with the highest flux of $12740 \mathrm{~L} \mathrm{~m}^{-2} \mathrm{~h}^{-1}$ for surfactant free oil/water emulsions and $6503 \mathrm{~L} \mathrm{~m}^{-2} \mathrm{~h}^{-1}$ for surfactant-stabilized emulsions with sundry organic solvents emulsions. Due to the porous architecture of NPM5 and high thermal insulation, the conductive heat loss to bulk water during sunlight illumination was reduced, thus increasing the solar energy efficiency. The PPy coated PVA membrane demonstrate the ability to absorb a wide range of wavelenghs $(250-1100 \mathrm{~nm})$ of sun light to thermal energy with an absorbance of over $99.9 \%$. Thanks to the low thermal conductivity of PVA, the thermal energy was restrained in the NPM, achieving high energy localization. Interestingly, the pores and gaps in between the PVA nanofibers serves as a 3D networks, where continuous water supply was provided to guarantee adequate water for solar vapor generation. The NPM solar steam system demonstrates a solar steam generation efficiency around $87.5 \%$ with an evaporation rate of $2.87 \mathrm{~kg} \mathrm{~m}^{-2} \mathrm{~h}^{-1}$ under 1 sun irradiation. The proof-ofconcept method toward oil/water separation and seawater desalination provides a possible 
solution for the real-life application of efficient, scalable oil/water separation, desalination and wastewater purification.

Despite excellent oil/water separation capability and solar vapor generation performance of the NPM, there are several drawbacks that remain for improvement in future works. The oil/water emulsion utilized for laboratorial separation testing is simplified. However, the industrial contaminated oily water has more complex composition and may contain microorganisms, heavy metals, organic dyes, etc. Thus, NPM may not meet the separation requirement in industrial settings. Besides, the anti-fouling performance under continuous operation is in urgent need, especially for highly viscous oil (e.g. crude oil). In the future work, real industrial contamination in the wastewater or crude oil spillage should be considered when preparing for oil/water mixture for testing. Another drawback in this work is the scalability of the membrane in industrial setting. At this point, electrospinning technique is matured in academic setting, but more consideration and adjustment should be taken to achieve more scalable, simple and cost-efficient manufacturing process. In addition, the two functions in NPM are realized in separate installations. Large-scale water transportation brings in excessive energy waste. Therefore, it is highly preferable to design a compact water purification system that combines the two functions together. The new water purification system would largely improve the water transportation efficiency as well as purification efficiency. 


\section{Reference}

1. Organization, W.H. and UNICEF, Progress on drinking water, sanitation and hygiene: 2017 update and SDG baselines. 2017.

2. Team, C.W., R.K. Pachauri, and L. Meyer, IPCC, 2014: climate change 2014: synthesis report. Contribution of Working Groups I. II and III to the Fifth Assessment Report of the intergovernmental panel on Climate Change. IPCC, Geneva, Switzerland, 2014. 151.

3. Fingas, M., Oil spill science and technology. 2016: Gulf professional publishing.

4. Kleindienst, S., J.H. Paul, and S.B. Joye, Using dispersants after oil spills: impacts on the composition and activity of microbial communities. Nature Reviews Microbiology, 2015. 13(6): p. 388.

5. Fujita, I., M. Yoshie, M. Mizutani, M. Sano, M. Fudo, and M. Tatsuguchi. An onboard vacuum suction spilled oil recovery system. in Oceans' 04 MTS/IEEE Techno-Ocean'04 (IEEE Cat. No. 04CH37600). 2004. IEEE.

6. Feng, L., Z. Zhang, Z. Mai, Y. Ma, B. Liu, L. Jiang, and D. Zhu, A super-hydrophobic and super-oleophilic coating mesh film for the separation of oil and water. Angewandte Chemie International Edition, 2004. 43(15): p. 2012-2014.

7. Wang, F., S. Lei, C. Li, J. Ou, M. Xue, and W. Li, Superhydrophobic Cu mesh combined with a superoleophilic polyurethane sponge for oil spill adsorption and collection. Industrial \& Engineering Chemistry Research, 2014. 53(17): p. 7141-7148.

8. Liang, W. and Z. Guo, Stable superhydrophobic and superoleophilic soft porous materials for oil/water separation. RSC Advances, 2013. 3(37): p. 16469-16474.

9. Yong, J., S.C. Singh, Z. Zhan, F. Chen, and C. Guo, How To Obtain Six Different Superwettabilities on a Same Microstructured Pattern: Relationship between Various Superwettabilities in Different Solid/Liquid/Gas Systems. Langmuir, 2019. 35(4): p. 921927.

10. Wang, C.-F., F.-S. Tzeng, H.-G. Chen, and C.-J. Chang, Ultraviolet-durable superhydrophobic zinc oxide-coated mesh films for surface and underwater-oil capture and transportation. Langmuir, 2012. 28(26): p. 10015-10019. 
11. Song, J., S. Huang, Y. Lu, X. Bu, J.E. Mates, A. Ghosh, R. Ganguly, C.J. Carmalt, I.P. Parkin, and $\mathrm{W} . \mathrm{Xu}$, Self-driven one-step oil removal from oil spill on water via selectivewettability steel mesh. ACS applied materials \& interfaces, 2014. 6(22): p. 19858-19865.

12. Dai, J., L. Wang, Y. Wang, S. Tian, X. Tian, A. Xie, R. Zhang, Y. Yan, and J. Pan, Robust Nacrelike Graphene Oxide-Calcium Carbonate Hybrid Mesh with Underwater Superoleophobic Property for Highly Efficient Oil/Water Separation. ACS Applied Materials \& Interfaces, 2020. 12(4): p. 4482-4493.

13. Shang, Y., Y. Si, A. Raza, L. Yang, X. Mao, B. Ding, and J. Yu, An in situ polymerization approach for the synthesis of superhydrophobic and superoleophilic nanofibrous membranes for oil-water separation. Nanoscale, 2012. 4(24): p. 7847-7854.

14. Calcagnile, P., D. Fragouli, I.S. Bayer, G.C. Anyfantis, L. Martiradonna, P.D. Cozzoli, R. Cingolani, and A. Athanassiou, Magnetically driven floating foams for the removal of oil contaminants from water. ACS nano, 2012. 6(6): p. 5413-5419.

15. Peng, L., H. Li, Y. Zhang, J. Su, P. Yu, and Y. Luo, A superhydrophobic 3D porous material for oil spill cleanup. RSC Advances, 2014. 4(87): p. 46470-46475.

16. Yin, Z., Y. Li, T. Song, M. Bao, Y. Li, J. Lu, and Y. Li, An environmentally benign approach to prepare superhydrophobic magnetic melamine sponge for effective oil/water separation. Separation and Purification Technology, 2020. 236: p. 116308.

17. Gao, X., L.P. Xu, Z. Xue, L. Feng, J. Peng, Y. Wen, S. Wang, and X. Zhang, Dualscaled porous nitrocellulose membranes with underwater superoleophobicity for highly efficient oil/water separation. Advanced Materials, 2014. 26(11): p. 1771-1775.

18. Ma, W., Y. Ding, M. Zhang, S. Gao, Y. Li, C. Huang, and G. Fu, Nature-inspired chemistry toward hierarchical superhydrophobic, antibacterial and biocompatible nanofibrous membranes for effective UV-shielding, self-cleaning and oil-water separation. Journal of hazardous materials, 2020. 384: p. 121476.

19. Li, T., H. Liu, X. Zhao, G. Chen, J. Dai, G. Pastel, C. Jia, C. Chen, E. Hitz, and D. Siddhartha, Scalable and Highly Efficient Mesoporous Wood-Based Solar Steam Generation Device: Localized Heat, Rapid Water Transport. Advanced Functional Materials, 2018. 28(16): p. 1707134. 
20. Zeng, X., L. Qian, X. Yuan, C. Zhou, Z. Li, J. Cheng, S. Xu, S. Wang, P. Pi, and X. Wen, Inspired by stenocara beetles: from water collection to high-efficiency water-in-oil emulsion separation. ACS nano, 2016. 11(1): p. 760-769.

21. Wang, Y., S. Luo, A. Chen, C. Shang, L. Peng, J. Shao, and Z. Liu, Environmentally friendly kaolin-coated meshes with superhydrophilicity and underwater superoleophobicity for oil/water separation. Separation and Purification Technology, 2020. 239: p. 116541.

22. Lewis, N.S., Research opportunities to advance solar energy utilization. Science, 2016. 351(6271): p. aad1920.

23. Su, Q., J. Zhang, and L.-Z. Zhang, Fouling resistance improvement with a new superhydrophobic electrospun PVDF membrane for seawater desalination. Desalination, 2020. 476: p. 114246.

24. Chu, S., Y. Cui, and N. Liu, The path towards sustainable energy. Nature materials, 2017. 16(1): p. 16.

25. Ni, G., G. Li, S.V. Boriskina, H. Li, W. Yang, T. Zhang, and G. Chen, Steam generation under one sun enabled by a floating structure with thermal concentration. Nature Energy, 2016. 1(9): p. 16126.

26. Sharshir, S.W., Y.M. Ellakany, and M.A. Eltawil, Exergoeconomic and environmental analysis of seawater desalination system augmented with nanoparticles and cotton hung pad. Journal of Cleaner Production, 2020. 248: p. 119180.

27. Ghasemi, H., G. Ni, A.M. Marconnet, J. Loomis, S. Yerci, N. Miljkovic, and G. Chen, Solar steam generation by heat localization. Nature communications, 2014. 5: p. 4449.

28. Dorji, P., D.I. Kim, S. Hong, S. Phuntsho, and H.K. Shon, Pilot-scale membrane capacitive deionisation for effective bromide removal and high water recovery in seawater desalination. Desalination, 2020. 479: p. 114309.

29. Shi, Z., W. Zhang, F. Zhang, X. Liu, D. Wang, J. Jin, and L. Jiang, Ultrafast separation of emulsified oil/water mixtures by ultrathin free-standing single-walled carbon nanotube network films. Advanced materials, 2013. 25(17): p. 2422-2427.

30. Zhang, W., Z. Shi, F. Zhang, X. Liu, J. Jin, and L. Jiang, Superhydrophobic and superoleophilic PVDF membranes for effective separation of water-in-oil emulsions with high flux. Advanced Materials, 2013. 25(14): p. 2071-2076. 
31. Qing, W., X. Shi, Y. Deng, W. Zhang, J. Wang, and C.Y. Tang, Robust superhydrophobic-superoleophilic polytetrafluoroethylene nanofibrous membrane for oil/water separation. Journal of Membrane Science, 2017. 540: p. 354-361.

32. Yao, X., J. Gao, Y. Song, and L. Jiang, Superoleophobic surfaces with controllable oil adhesion and their application in oil transportation. Advanced Functional Materials, 2011. 21(22): p. 4270-4276.

33. Zhang, F., W.B. Zhang, Z. Shi, D. Wang, J. Jin, and L. Jiang, Nanowire-haired inorganic membranes with superhydrophilicity and underwater ultralow adhesive superoleophobicity for high-efficiency oil/water separation. Advanced Materials, 2013. 25(30): p. 4192-4198.

34. He, K., H. Duan, G.Y. Chen, X. Liu, W. Yang, and D. Wang, Cleaning of oil fouling with water enabled by zwitterionic polyelectrolyte coatings: overcoming the imperative challenge of oil-water separation membranes. ACS nano, 2015. 9(9): p. 9188-9198.

35. Yan, S., Y. Li, F. Xie, J. Wu, X. Jia, J. Yang, H. Song, and Z. Zhang, Environmentally Safe and Porous MS@TiO2@PPy Monolithswith Superior Visible-Light Photocatalytic Properties for Rapid Oil-Water Separation and Water Purification. ACS Sustainable Chemistry \& Engineering, 2020. 8(13): p. 5347-5359.

36. Zhang, W., Y. Zhu, X. Liu, D. Wang, J. Li, L. Jiang, and J. Jin, Salt-induced fabrication of superhydrophilic and underwater superoleophobic PAA-g-PVDF membranes for effective separation of oil-in-water emulsions. Angewandte Chemie International Edition, 2014. 53(3): p. 856-860.

37. Gong, Z., N. Yang, Z. Chen, B. Jiang, Y. Sun, X. Yang, and L. Zhang, Fabrication of meshes with inverse wettability based on the TiO2 nanowires for continuous oil/water separation. Chemical Engineering Journal, 2020. 380: p. 122524.

38. Tian, D., X. Zhang, Y. Tian, Y. Wu, X. Wang, J. Zhai, and L. Jiang, Photo-induced water-oil separation based on switchable superhydrophobicity-superhydrophilicity and underwater superoleophobicity of the aligned $\mathrm{ZnO}$ nanorod array-coated mesh films. Journal of Materials Chemistry, 2012. 22(37): p. 19652-19657. 
39. Han, K., L. Heng, and L. Jiang, Multiphase media antiadhesive coatings: Hierarchical Self-Assembled porous materials generated using breath figure patterns. ACS nano, 2016. 10(12): p. 11087-11095.

40. Gao, S., J. Sun, P. Liu, F. Zhang, W. Zhang, S. Yuan, J. Li, and J. Jin, A robust polyionized hydrogel with an unprecedented underwater anti-crude-oil-adhesion property. Advanced Materials, 2016. 28(26): p. 5307-5314.

41. Xu, L.P., J. Zhao, B. Su, X. Liu, J. Peng, Y. Liu, H. Liu, G. Yang, L. Jiang, and Y. Wen, An Ion - Induced Low - Oil - Adhesion Organic/Inorganic Hybrid Film for Stable Superoleophobicity in Seawater. Advanced materials, 2013. 25(4): p. 606-611.

42. Zhou, L., Y. Tan, J. Wang, W. Xu, Y. Yuan, W. Cai, S. Zhu, and J. Zhu, 3D selfassembly of aluminium nanoparticles for plasmon-enhanced solar desalination. Nature Photonics, 2016. 10(6): p. 393.

43. Pal, A., G. Natu, K. Ahmad, and A. Chattopadhyay, Phosphorus induced crystallinity in carbon dots for solar light assisted seawater desalination. Journal of Materials Chemistry A, 2018. 6(9): p. 4111-4118.

44. Yao, J. and G. Yang, An efficient solar-enabled 2D layered alloy material evaporator for seawater desalination. Journal of Materials Chemistry A, 2018. 6(9): p. 3869-3876.

45. Chen, C., Y. Li, J. Song, Z. Yang, Y. Kuang, E. Hitz, C. Jia, A. Gong, F. Jiang, and J. Zhu, Highly flexible and efficient solar steam generation device. Advanced Materials, 2017. 29(30): p. 1701756.

46. Zhu, M., Y. Li, F. Chen, X. Zhu, J. Dai, Y. Li, Z. Yang, X. Yan, J. Song, and Y. Wang, Plasmonic Wood for High-Efficiency Solar Steam Generation. Advanced Energy Materials, 2018. 8(4): p. 1701028.

47. Liu, T. and Y. Li, Photocatalysis: Plasmonic solar desalination. Nature Photonics, 2016. 10(6): p. 361.

48. Ye, M., J. Jia, Z. Wu, C. Qian, R. Chen, P.G. O'Brien, W. Sun, Y. Dong, and G.A. Ozin, Synthesis of black TiOx nanoparticles by Mg reduction of TiO2 nanocrystals and their application for solar water evaporation. Advanced Energy Materials, 2017. 7(4): p. 1601811. 
49. Yao, J., Z. Zheng, and G. Yang, Layered tin monoselenide as advanced photothermal conversion materials for efficient solar energy-driven water evaporation. Nanoscale, 2018. 10(6): p. 2876-2886.

50. Xu, N., X. Hu, W. Xu, X. Li, L. Zhou, S. Zhu, and J. Zhu, Mushrooms as efficient solar steam-generation devices. Advanced Materials, 2017. 29(28): p. 1606762.

51. Ren, H., M. Tang, B. Guan, K. Wang, J. Yang, F. Wang, M. Wang, J. Shan, Z. Chen, and D. Wei, Hierarchical graphene foam for efficient omnidirectional solar-thermal energy conversion. Advanced materials, 2017. 29(38): p. 1702590.

52. Morciano, M., M. Fasano, U. Salomov, L. Ventola, E. Chiavazzo, and P. Asinari, Efficient steam generation by inexpensive narrow gap evaporation device for solar applications. Scientific reports, 2017. 7(1): p. 11970.

53. Carswell, C., Unique oil spill in East China Sea frustrates scientists. Nature, 2018. 554(7690).

54. Genzer, J. and K. Efimenko, Recent developments in superhydrophobic surfaces and their relevance to marine fouling: a review. Biofouling, 2006. 22(5): p. 339-360.

55. Chen, D.-L., J.-W. Mao, Z.-D. Chen, K.-X. Yu, D.-D. Han, and H.-B. Sun, Fabrication of bionic reed leaf superhydrophobic surface by laser processing. Chinese Science Bulletin, 2018. 64(12): p. 1303-1308.

56. Yong, J., F. Chen, Q. Yang, J. Huo, and X. Hou, Superoleophobic surfaces. Chemical Society Reviews, 2017. 46(14): p. 4168-4217.

57. Wang, S. and L. Jiang, Definition of superhydrophobic states. Advanced Materials, 2007. 19(21): p. 3423-3424.

58. Wen, G., Z. Guo, and W. Liu, Biomimetic polymeric superhydrophobic surfaces and nanostructures: from fabrication to applications. Nanoscale, 2017. 9(10): p. 3338-3366.

59. Si, Y. and Z. Guo, Superhydrophobic nanocoatings: from materials to fabrications and to applications. Nanoscale, 2015. 7(14): p. 5922-5946.

60. Cassie, A. and S. Baxter, Wettability of porous surfaces. Transactions of the Faraday society, 1944. 40: p. 546-551.

61. Wenzel, R.N., Resistance of solid surfaces to wetting by water. Industrial \& Engineering Chemistry, 1936. 28(8): p. 988-994. 
62. Liu, M. and L. Jiang, Switchable adhesion on liquid/solid interfaces. Advanced Functional Materials, 2010. 20(21): p. 3753-3764.

63. Xia, F. and L. Jiang, Bio-inspired, smart, multiscale interfacial materials. Advanced materials, 2008. 20(15): p. 2842-2858.

64. Erbil, H.Y. and C.E. Cansoy, Range of applicability of the Wenzel and Cassie- Baxter equations for superhydrophobic surfaces. Langmuir, 2009. 25(24): p. 14135-14145.

65. Yong, J., Q. Yang, F. Chen, D. Zhang, G. Du, H. Bian, J. Si, F. Yun, and X. Hou, Superhydrophobic PDMS surfaces with three-dimensional (3D) pattern-dependent controllable adhesion. Applied Surface Science, 2014. 288: p. 579-583.

66. Cheng, Z., M. Du, H. Lai, N. Zhang, and K. Sun, From petal effect to lotus effect: a facile solution immersion process for the fabrication of super-hydrophobic surfaces with controlled adhesion. Nanoscale, 2013. 5(7): p. 2776-2783.

67. Li, K., X. Zeng, H. Li, X. Lai, C. Ye, and H. Xie, Study on the wetting behavior and theoretical models of polydimethylsiloxane/silica coating. Applied Surface Science, 2013. 279: p. 458-463.

68. Li, J., Z. Jing, F. Zha, Y. Yang, Q. Wang, and Z. Lei, Facile spray-coating process for the fabrication of tunable adhesive superhydrophobic surfaces with heterogeneous chemical compositions used for selective transportation of microdroplets with different volumes. ACS applied materials \& interfaces, 2014. 6(11): p. 8868-8877.

69. Liu, M., S. Wang, Z. Wei, Y. Song, and L. Jiang, Bioinspired design of a superoleophobic and low adhesive water/solid interface. Advanced Materials, 2009. 21(6): p. 665-669.

70. Liu, X., J. Gao, Z. Xue, L. Chen, L. Lin, L. Jiang, and S. Wang, Bioinspired oil strider floating at the oil/water interface supported by huge superoleophobic force. ACS nano, 2012. 6(6): p. 5614-5620.

71. Wu, D., S.-z. Wu, Q.-D. Chen, S. Zhao, H. Zhang, J. Jiao, J.A. Piersol, J.-N. Wang, H.-B. Sun, and L. Jiang, Facile creation of hierarchical PDMS microstructures with extreme underwater superoleophobicity for anti-oil application in microfluidic channels. Lab on a Chip, 2011. 11(22): p. 3873-3879.

72. Zhang, C.-L. and S.-H. Yu, Spraying functional fibres by electrospinning. Materials Horizons, 2016. 3(4): p. 266-269. 
73. Agarwal, S., A. Greiner, and J.H. Wendorff, Functional materials by electrospinning of polymers. Progress in Polymer Science, 2013. 38(6): p. 963-991.

74. Di, J., Y. Zhao, and J. Yu, Fabrication of molecular sieve fibers by electrospinning. Journal of Materials Chemistry, 2011. 21(24): p. 8511-8520.

75. Reneker, D.H., A.L. Yarin, H. Fong, and S. Koombhongse, Bending instability of electrically charged liquid jets of polymer solutions in electrospinning. Journal of Applied physics, 2000. 87(9): p. 4531-4547.

76. Reneker, D.H. and A.L. Yarin, Electrospinning jets and polymer nanofibers. Polymer, 2008. 49(10): p. 2387-2425.

77. Ahmed, F.E., B.S. Lalia, and R. Hashaikeh, A review on electrospinning for membrane fabrication: challenges and applications. Desalination, 2015. 356: p. 15-30.

78. Gao, H., Y. Yang, O. Akampumuza, J. Hou, H. Zhang, and X. Qin, A low filtration resistance three-dimensional composite membrane fabricated via free surface electrospinning for effective PM 2.5 capture. Environmental Science: Nano, 2017. 4(4): p. 864-875.

79. Acatay, K., E. Simsek, C. Ow - Yang, and Y.Z. Menceloglu, Tunable, superhydrophobically stable polymeric surfaces by electrospinning. Angewandte Chemie International Edition, 2004. 43(39): p. 5210-5213.

80. Jiang, L., Y. Zhao, and J. Zhai, A lotus-leaf-like superhydrophobic surface: a porous microsphere/nanofiber composite film prepared by electrohydrodynamics. Angewandte Chemie International Edition, 2004. 43(33): p. 4338-4341.

81. Yang, G., X. Li, Y. He, J. Ma, G. Ni, and S. Zhou, From nano to micro to macro: electrospun hierarchically structured polymeric fibers for biomedical applications. Progress in Polymer Science, 2018. 81: p. 80-113.

82. Wang, X., B. Ding, J. Yu, and M. Wang, Engineering biomimetic superhydrophobic surfaces of electrospun nanomaterials. Nano today, 2011. 6(5): p. 510-530.

83. Gao, S., X. Dong, J. Huang, J. Dong, Y. Cheng, Z. Chen, and Y. Lai, Co-solvent induced self-roughness superhydrophobic coatings with self-healing property for versatile oilwater separation. Applied Surface Science, 2018. 459: p. 512-519. 
84. Zhang, J., F. Zhang, J. Song, L. Liu, Y. Si, J. Yu, and B. Ding, Electrospun flexible nanofibrous membranes for oil/water separation. Journal of Materials Chemistry A, 2019. 7(35): p. 20075-20102.

85. Cassie, A., Contact angles. Discussions of the Faraday society, 1948. 3: p. 11-16.

86. Cao, L., Q. Fu, Y. Si, B. Ding, and J. Yu, Porous materials for sound absorption. Composites Communications, 2018. 10: p. 25-35.

87. Bhardwaj, N. and S.C. Kundu, Electrospinning: a fascinating fiber fabrication technique. Biotechnology advances, 2010. 28(3): p. 325-347.

88. Nuraje, N., W.S. Khan, Y. Lei, M. Ceylan, and R. Asmatulu, Superhydrophobic electrospun nanofibers. Journal of Materials Chemistry A, 2013. 1(6): p. 1929-1946.

89. Wang, Z., M. Elimelech, and S. Lin, Environmental applications of interfacial materials with special wettability. Environmental science \& technology, 2016. 50(5): p. 2132-2150.

90. Doshi, B., M. Sillanpää, and S. Kalliola, A review of bio-based materials for oil spill treatment. Water research, 2018. 135: p. 262-277.

91. Li, F., Z. Wang, S. Huang, Y. Pan, and X. Zhao, Flexible, durable, and unconditioned superoleophobic/superhydrophilic surfaces for controllable transport and oil-water separation. Advanced Functional Materials, 2018. 28(20): p. 1706867.

92. Xue, Z., Y. Cao, N. Liu, L. Feng, and L. Jiang, Special wettable materials for oil/water separation. Journal of Materials Chemistry A, 2014. 2(8): p. 2445-2460.

93. Ge, J., Q. Jin, D. Zong, J. Yu, and B. Ding, Biomimetic multilayer nanofibrous membranes with elaborated superwettability for effective purification of emulsified oily wastewater. ACS applied materials \& interfaces, 2018. 10(18): p. 16183-16192.

94. Wang, X., J. Yu, G. Sun, and B. Ding, Electrospun nanofibrous materials: a versatile medium for effective oil/water separation. Materials today, 2016. 19(7): p. 403-414.

95. Wang, X., B. Ding, G. Sun, M. Wang, and J. Yu, Electro-spinning/netting: A strategy for the fabrication of three-dimensional polymer nano-fiber/nets. Progress in Materials Science, 2013. 58(8): p. 1173-1243.

96. Hong, S.K., S. Bae, H. Jeon, M. Kim, S.J. Cho, and G. Lim, An underwater superoleophobic nanofibrous cellulosic membrane for oil/water separation with high separation flux and high chemical stability. Nanoscale, 2018. 10(6): p. 3037-3045. 
97. Li, Z., C.M. Tan, W. Tio, J. Ang, and D.D. Sun, Manta ray gill inspired radially distributed nanofibrous membrane for efficient and continuous oil-water separation. Environmental Science: Nano, 2018. 5(6): p. 1466-1472.

98. You, H., X. Li, Y. Yang, B. Wang, Z. Li, X. Wang, M. Zhu, and B.S. Hsiao, High flux low pressure thin film nanocomposite ultrafiltration membranes based on nanofibrous substrates. Separation and Purification Technology, 2013. 108: p. 143-151.

99. You, H., Y. Yang, X. Li, K. Zhang, X. Wang, M. Zhu, and B.S. Hsiao, Low pressure high flux thin film nanofibrous composite membranes prepared by electrospraying technique combined with solution treatment. Journal of membrane science, 2012. 394: p. 241-247.

100. Wu, J.-X., J. Zhang, Y.-L. Kang, G. Wu, S.-C. Chen, and Y.-Z. Wang, Reusable and recyclable superhydrophilic electrospun nanofibrous membranes with in situ co-crosslinked polymer-chitin nanowhisker network for robust oil-in-water emulsion separation. ACS Sustainable Chemistry \& Engineering, 2018. 6(2): p. 1753-1762.

101. Zhu, M., D. Hua, H. Pan, F. Wang, B. Manshian, S.J. Soenen, R. Xiong, and C. Huang, Green electrospun and crosslinked poly (vinyl alcohol)/poly (acrylic acid) composite membranes for antibacterial effective air filtration. Journal of colloid and interface science, 2018. 511: p. 411-423.

102. Zang, L., J. Ma, D. Lv, Q. Liu, W. Jiao, and P. Wang, A core-shell fiber-constructed $p H-$ responsive nanofibrous hydrogel membrane for efficient oil/water separation. Journal of Materials Chemistry A, 2017. 5(36): p. 19398-19405.

103. Chen, S., C. Lv, K. Hao, L. Jin, Y. Xie, W. Zhao, S. Sun, X. Zhang, and C. Zhao, Multifunctional negatively-charged poly (ether sulfone) nanofibrous membrane for water remediation. Journal of colloid and interface science, 2019. 538: p. 648-659.

104. Ge, J., J. Zhang, F. Wang, Z. Li, J. Yu, and B. Ding, Superhydrophilic and underwater superoleophobic nanofibrous membrane with hierarchical structured skin for effective oil-in-water emulsion separation. Journal of Materials Chemistry A, 2017. 5(2): p. 497502.

105. Jayaramulu, K., F. Geyer, M. Petr, R. Zboril, D. Vollmer, and R.A. Fischer, Shape controlled hierarchical porous hydrophobic/oleophilic metal-organic nanofibrous gel composites for oil adsorption. Advanced Materials, 2017. 29(12): p. 1605307. 
106. Cai, Y., D. Chen, N. Li, Q. Xu, H. Li, J. He, and J. Lu, Nanofibrous metal-organic framework composite membrane for selective efficient oil/water emulsion separation. Journal of Membrane Science, 2017. 543: p. 10-17.

107. Zhu, Z., W. Wang, D. Qi, Y. Luo, Y. Liu, Y. Xu, F. Cui, C. Wang, and X. Chen, Calcinable Polymer Membrane with Revivability for Efficient Oily-Water Remediation. Advanced Materials, 2018. 30(30): p. 1801870.

108. Liao, K., X.Y. Ye, P.C. Chen, and Z.K. Xu, Biomineralized polypropylene/CaCO3 composite nonwoven meshes for oil/water separation. Journal of Applied Polymer Science, 2014. 131(4).

109. Li, X., M. Wang, C. Wang, C. Cheng, and X. Wang, Facile immobilization of Ag nanocluster on nanofibrous membrane for oil/water separation. ACS applied materials \& interfaces, 2014. 6(17): p. 15272-15282.

110. Liu, L. and W. Yuan, A hierarchical functionalized biodegradable PLA electrospun nanofibrous membrane with superhydrophobicity and antibacterial properties for oil/water separation. New Journal of Chemistry, 2018. 42(21): p. 17615-17624.

111. Shami, Z., S.M. Amininasab, and P. Shakeri, Structure-property relationships of nanosheeted 3D hierarchical roughness MgAl-layered double hydroxide branched to an electrospun porous nanomembrane: a superior oil-removing nanofabric. ACS applied materials \& interfaces, 2016. 8(42): p. 28964-28973.

112. Hare, E., E. Shafrin, and W. Zisman, Properties of films of adsorbed fluorinated acids. The Journal of physical chemistry, 1954. 58(3): p. 236-239.

113. Lee, M.W., S. An, S.S. Latthe, C. Lee, S. Hong, and S.S. Yoon, Electrospun polystyrene nanofiber membrane with superhydrophobicity and superoleophilicity for selective separation of water and low viscous oil. ACS applied materials \& interfaces, 2013. 5(21): p. $10597-10604$.

114. Song, B. and Q. Xu, Highly hydrophobic and superoleophilic nanofibrous mats with controllable pore sizes for efficient oil/water separation. Langmuir, 2016. 32(39): p. 9960-9966.

115. Obaid, M., N.A. Barakat, O. Fadali, M. Motlak, A.A. Almajid, and K.A. Khalil, Effective and reusable oil/water separation membranes based on modified polysulfone electrospun nanofiber mats. Chemical engineering journal, 2015. 259: p. 449-456. 
116. Liu, Z., J.-h. Zhao, P. Liu, and J.-h. He, Tunable surface morphology of electrospun PMMA fiber using binary solvent. Applied Surface Science, 2016. 364: p. 516-521.

117. Tijing, L.D., M.T.G. Ruelo, A. Amarjargal, H.R. Pant, C.-H. Park, D.W. Kim, and C.S. Kim, Antibacterial and superhydrophilic electrospun polyurethane nanocomposite fibers containing tourmaline nanoparticles. Chemical Engineering Journal, 2012. 197: p. 41-48.

118. Zhu, H., S. Qiu, W. Jiang, D. Wu, and C. Zhang, Evaluation of electrospun polyvinyl chloride/polystyrene fibers as sorbent materials for oil spill cleanup. Environmental science \& technology, 2011. 45(10): p. 4527-4531.

119. Kaplan, J.A., H. Lei, R. Liu, R. Padera, Y.L. Colson, and M.W. Grinstaff, Imparting superhydrophobicity to biodegradable poly (lactide-co-glycolide) electrospun meshes. Biomacromolecules, 2014. 15(7): p. 2548-2554.

120. Jing, Y., L. Zhang, R. Huang, D. Bai, H. Bai, Q. Zhang, and Q. Fu, Ultrahighperformance electrospun polylactide membranes with excellent oil/water separation ability via interfacial stereocomplex crystallization. Journal of Materials Chemistry A, 2017. 5(37): p. 19729-19737.

121. Reshmi, C., S.P. Sundaran, A. Juraij, and S. Athiyanathil, Fabrication of superhydrophobic polycaprolactone/beeswax electrospun membranes for high-efficiency oil/water separation. RSC advances, 2017. 7(4): p. 2092-2102.

122. Zhao, J., W. Wang, C. Ye, Y. Li, and J. You, Gravity-driven ultrafast separation of water-in-oil emulsion by hierarchically porous electrospun Poly (L-lactide) fabrics. Journal of membrane science, 2018. 563: p. 762-767.

123. Tenjimbayashi, M., K. Sasaki, T. Matsubayashi, J. Abe, K. Manabe, S. Nishioka, and S. Shiratori, A biologically inspired attachable, self-standing nanofibrous membrane for versatile use in oil-water separation. Nanoscale, 2016. 8(21): p. 10922-10927.

124. Qiu, S., L. Hou, J. Liu, F. Guo, Y. Zhang, L. Zhang, K. Liu, N. Wang, and Y. Zhao, High-flux, continuous oil spill collection by using a hydrophobic/oleophilic nanofibrous container. RSC advances, 2017. 7(32): p. 19434-19438.

125. Chu, Z. and S. Seeger, Superamphiphobic surfaces. Chemical Society Reviews, 2014. 43(8): p. 2784-2798.

126. Zhang, C., P. Li, and B. Cao, Electrospun microfibrous membranes based on PIM1/POSS with high oil wettability for separation of oil-water mixtures and cleanup of oil 
soluble contaminants. Industrial \& Engineering Chemistry Research, 2015. 54(35): p. 8772-8781.

127. Ma, W., Z. Guo, J. Zhao, Q. Yu, F. Wang, J. Han, H. Pan, J. Yao, Q. Zhang, and S.K. Samal, Polyimide/cellulose acetate core/shell electrospun fibrous membranes for oilwater separation. Separation and Purification Technology, 2017. 177: p. 71-85.

128. Ma, W., Q. Zhang, S.K. Samal, F. Wang, B. Gao, H. Pan, H. Xu, J. Yao, X. Zhan, and S.C. De Smedt, Core-sheath structured electrospun nanofibrous membranes for oilwater separation. Rsc Advances, 2016. 6(48): p. 41861-41870.

129. Wang, Y., K. Ma, and J.H. Xin, Liquid Manipulation: Stimuli-Responsive Bioinspired Materials for Controllable Liquid Manipulation: Principles, Fabrication, and Applications (Adv. Funct. Mater. 6/2018). Advanced Functional Materials, 2018. 28(6): p. 1870039.

130. Cheng, B., Z. Li, Q. Li, J. Ju, W. Kang, and M. Naebe, Development of smart poly (vinylidene fluoride)-graft-poly (acrylic acid) tree-like nanofiber membrane for $\mathrm{pH}$ responsive oil/water separation. Journal of membrane science, 2017. 534: p. 1-8.

131. Li, J.-J., Y.-N. Zhou, Z.-D. Jiang, and Z.-H. Luo, Electrospun fibrous mat with pHswitchable superwettability that can separate layered oil/water mixtures. Langmuir, 2016. 32(50): p. 13358-13366.

132. Che, H., M. Huo, L. Peng, T. Fang, N. Liu, L. Feng, Y. Wei, and J. Yuan, CO2 responsive nanofibrous membranes with switchable oil/water wettability. Angewandte Chemie International Edition, 2015. 54(31): p. 8934-8938.

133. Li, J.-J., L.-T. Zhu, and Z.-H. Luo, Electrospun fibrous membrane with enhanced swithchable oil/water wettability for oily water separation. Chemical Engineering Journal, 2016. 287: p. 474-481.

134. Cai, Y., D. Chen, N. Li, Q. Xu, H. Li, J. He, and J. Lu, A facile method to fabricate a double-layer stainless steel mesh for effective separation of water-in-oil emulsions with high flux. Journal of Materials Chemistry A, 2016. 4(48): p. 18815-18821.

135. Li, J.-J., Y.-N. Zhou, and Z.-H. Luo, Smart fiber membrane for $\mathrm{pH}$-induced oil/water separation. ACS applied materials \& interfaces, 2015. 7(35): p. 19643-19650. 
136. Zeng, J., C. Lv, G. Liu, Z. Zhang, Z. Dong, J. Liu, and Y. Wang, A novel ion-imprinted membrane induced by amphiphilic block copolymer for selective separation of Pt (IV) from aqueous solutions. Journal of membrane science, 2019. 572: p. 428-441.

137. Li, Y., L. Zhu, N. Grishkewich, K.C. Tam, J. Yuan, Z. Mao, and X. Sui, CO2-responsive cellulose nanofibers aerogels for switchable oil-water separation. ACS applied materials \& interfaces, 2019. 11(9): p. 9367-9373.

138. Tian, J., B. Huang, C. Xiao, and P. Vana, Intelligent CO 2-and photo-dual-responsive polymer vesicles with tunable wall thickness. Polymer Chemistry, 2019. 10(13): p. 16101618.

139. Tiwari, G., A. Kumar, and M. Sodha, A review—cooling by water evaporation over roof. Energy conversion and Management, 1982. 22(2): p. 143-153.

140. Tao, P., G. Ni, C. Song, W. Shang, J. Wu, J. Zhu, G. Chen, and T. Deng, Solar-driven interfacial evaporation. Nature energy, 2018. 3(12): p. 1031-1041.

141. Chen, C., Y. Kuang, and L. Hu, Challenges and opportunities for solar evaporation. Joule, 2019.

142. Blanco, J., S. Malato, P. Fernández-Ibañez, D. Alarcón, W. Gernjak, and M. Maldonado, Review of feasible solar energy applications to water processes. Renewable and Sustainable Energy Reviews, 2009. 13(6-7): p. 1437-1445.

143. Mistry, K.H., M.A. Antar, and J.H. Lienhard V, An improved model for multiple effect distillation. Desalination and Water Treatment, 2013. 51(4-6): p. 807-821.

144. Ohmura, A. and M. Wild, Is the hydrological cycle accelerating? Science, 2002. 298(5597): p. 1345-1346.

145. Lee, J., T. Laoui, and R. Karnik, Nanofluidic transport governed by the liquid/vapour interface. Nature nanotechnology, 2014. 9(4): p. 317.

146. Liu, Z., H. Song, D. Ji, C. Li, A. Cheney, Y. Liu, N. Zhang, X. Zeng, B. Chen, and J. Gao, Extremely cost-effective and efficient solar vapor generation under nonconcentrated illumination using thermally isolated black paper. Global Challenges, 2017. 1(2): p. 1600003.

147. Li, X., W. Xu, M. Tang, L. Zhou, B. Zhu, S. Zhu, and J. Zhu, Graphene oxide-based efficient and scalable solar desalination under one sun with a confined $2 D$ water path. Proceedings of the National Academy of Sciences, 2016. 113(49): p. 13953-13958. 
148. Zielinski, M.S., J.-W. Choi, T. La Grange, M. Modestino, S.M.H. Hashemi, Y. Pu, S. Birkhold, J.A. Hubbell, and D. Psaltis, Hollow mesoporous plasmonic nanoshells for enhanced solar vapor generation. Nano letters, 2016. 16(4): p. 2159-2167.

149. Neumann, O., C. Feronti, A.D. Neumann, A. Dong, K. Schell, B. Lu, E. Kim, M. Quinn, S. Thompson, and N. Grady, Compact solar autoclave based on steam generation using broadband light-harvesting nanoparticles. Proceedings of the National Academy of Sciences, 2013. 110(29): p. 11677-11681.

150. Bellich, B., M. Borgogna, M. Cok, and A. Cesàro, Water evaporation from gel beads. Journal of thermal analysis and calorimetry, 2011. 103(1): p. 81-88.

151. Zhao, F., Y. Guo, X. Zhou, W. Shi, and G. Yu, Materials for solar-powered water evaporation. Nature Reviews Materials, 2020: p. 1-14.

152. Chen, C., L. Zhou, J. Yu, Y. Wang, S. Nie, S. Zhu, and J. Zhu, Dual functional asymmetric plasmonic structures for solar water purification and pollution detection. Nano Energy, 2018. 51: p. 451-456.

153. Zhou, L., Y. Tan, D. Ji, B. Zhu, P. Zhang, J. Xu, Q. Gan, Z. Yu, and J. Zhu, Selfassembly of highly efficient, broadband plasmonic absorbers for solar steam generation. Science advances, 2016. 2(4): p. e1501227.

154. Wang, J., Y. Li, L. Deng, N. Wei, Y. Weng, S. Dong, D. Qi, J. Qiu, X. Chen, and T. Wu, High-performance photothermal conversion of narrow-bandgap Ti2O3 nanoparticles. Advanced Materials, 2017. 29(3): p. 1603730.

155. Liu, H., C. Chen, H. Wen, R. Guo, N.A. Williams, B. Wang, F. Chen, and L. Hu, Narrow bandgap semiconductor decorated wood membrane for high-efficiency solar-assisted water purification. Journal of Materials Chemistry A, 2018. 6(39): p. 18839-18846.

156. Zhang, P., F. Liu, Q. Liao, H. Yao, H. Geng, H. Cheng, C. Li, and L. Qu, $A$ microstructured graphene/poly (N-isopropylacrylamide) membrane for intelligent solar water evaporation. Angewandte Chemie International Edition, 2018. 57(50): p. 1634316347.

157. Li, Y., T. Gao, Z. Yang, C. Chen, W. Luo, J. Song, E. Hitz, C. Jia, Y. Zhou, and B. Liu, 3D-printed, all-in-one evaporator for high-efficiency solar steam generation under 1 sun illumination. Advanced materials, 2017. 29(26): p. 1700981. 
158. Zhou, X., F. Zhao, Y. Guo, Y. Zhang, and G. Yu, A hydrogel-based antifouling solar evaporator for highly efficient water desalination. Energy \& Environmental Science, 2018. 11(8): p. 1985-1992.

159. Cui, S., H. Liu, L. Gan, Y. Li, and D. Zhu, Fabrication of low-dimension nanostructures based on organic conjugated molecules. Advanced Materials, 2008. 20(15): p. 2918-2925.

160. Xu, L., L. Cheng, C. Wang, R. Peng, and Z. Liu, Conjugated polymers for photothermal therapy of cancer. Polymer Chemistry, 2014. 5(5): p. 1573-1580.

161. Wang, Y., Y. Shi, L. Pan, Y. Ding, Y. Zhao, Y. Li, Y. Shi, and G. Yu, Dopant-enabled supramolecular approach for controlled synthesis of nanostructured conductive polymer hydrogels. Nano letters, 2015. 15(11): p. 7736-7741.

162. Shi, Y., M. Wang, C. Ma, Y. Wang, X. Li, and G. Yu, A conductive self-healing hybrid gel enabled by metal-ligand supramolecule and nanostructured conductive polymer. Nano letters, 2015. 15(9): p. 6276-6281.

163. Liu, J., J. Geng, L.-D. Liao, N. Thakor, X. Gao, and B. Liu, Conjugated polymer nanoparticles for photoacoustic vascular imaging. Polymer Chemistry, 2014. 5(8): p. 2854-2862.

164. Gibson, G.L., T.M. McCormick, and D.S. Seferos, Atomistic band gap engineering in donor-acceptor polymers. Journal of the American Chemical Society, 2012. 134(1): p. 539-547.

165. Chen, M., X. Fang, S. Tang, and N. Zheng, Polypyrrole nanoparticles for highperformance in vivo near-infrared photothermal cancer therapy. Chemical communications, 2012. 48(71): p. 8934-8936.

166. Bjorklund, R.B. and B. Liedberg, Electrically conducting composites of colloidal polypyrrole and methylcellulose. Journal of the Chemical Society, Chemical Communications, 1986(16): p. 1293-1295.

167. Yang, K., H. Xu, L. Cheng, C. Sun, J. Wang, and Z. Liu, In vitro and in vivo nearinfrared photothermal therapy of cancer using polypyrrole organic nanoparticles. Advanced materials, 2012. 24(41): p. 5586-5592.

168. Zha, Z., X. Yue, Q. Ren, and Z. Dai, Uniform polypyrrole nanoparticles with high photothermal conversion efficiency for photothermal ablation of cancer cells. Advanced materials, 2013. 25(5): p. 777-782. 
169. Gong, H., L. Cheng, J. Xiang, H. Xu, L. Feng, X. Shi, and Z. Liu, Near-infrared absorbing polymeric nanoparticles as a versatile drug carrier for cancer combination therapy. Advanced Functional Materials, 2013. 23(48): p. 6059-6067.

170. Jiang, Q., H.G. Derami, D. Ghim, S. Cao, Y.-S. Jun, and S. Singamaneni, Polydopaminefilled bacterial nanocellulose as a biodegradable interfacial photothermal evaporator for highly efficient solar steam generation. Journal of Materials Chemistry A, 2017. 5(35): p. 18397-18402.

171. Chen, Q., Z. Pei, Y. Xu, Z. Li, Y. Yang, Y. Wei, and Y. Ji, A durable monolithic polymer foam for efficient solar steam generation. Chemical science, 2018. 9(3): p. 623-628.

172. Zhao, Y. and C. Burda, Development of plasmonic semiconductor nanomaterials with copper chalcogenides for a future with sustainable energy materials. Energy \& Environmental Science, 2012. 5(2): p. 5564-5576.

173. Yu, F., Y. Chen, X. Liang, J. Xu, C. Lee, Q. Liang, P. Tao, and T. Deng, Dispersion stability of thermal nanofluids. Progress in natural science: Materials International, 2017. 27(5): p. 531-542.

174. Umlauff, M., J. Hoffmann, H. Kalt, W. Langbein, J.M. Hvam, M. Scholl, J. Söllner, M. Heuken, B. Jobst, and D. Hommel, Direct observation of free-exciton thermalization in quantum-well structures. Physical Review B, 1998. 57(3): p. 1390.

175. Whang, A.J.-W., Y.-Y. Chen, and B.-Y. Wu, Innovative design of cassegrain solar concentrator system for indoor illumination utilizing chromatic aberration to filter out ultraviolet and infrared in sunlight. Solar Energy, 2009. 83(8): p. 1115-1122.

176. Choi, W., A. Termin, and M.R. Hoffmann, The role of metal ion dopants in quantumsized TiO2: correlation between photoreactivity and charge carrier recombination dynamics. The Journal of Physical Chemistry, 2002. 98(51): p. 13669-13679.

177. Dette, C., M.A. Pérez-Osorio, C.S. Kley, P. Punke, C.E. Patrick, P. Jacobson, F. Giustino, S.J. Jung, and $\mathrm{K}$. Kern, TiO2 anatase with a bandgap in the visible region. Nano letters, 2014. 14(11): p. 6533-6538.

178. Tao, J., T. Luttrell, and M. Batzill, A two-dimensional phase of TiO 2 with a reduced bandgap. Nature chemistry, 2011. 3(4): p. 296-300.

179. Li, Y., Y. Yang, X. Shu, D. Wan, N. Wei, X. Yu, M.B. Breese, T. Venkatesan, J.M. Xue, and Y. Liu, From Titanium Sesquioxide to Titanium Dioxide: Oxidation-Induced 
Structural, Phase, and Property Evolution. Chemistry of Materials, 2018. 30(13): p. 4383-4392.

180. Zhao, Q., T. Fan, J. Ding, D. Zhang, Q. Guo, and M. Kamada, Super black and ultrathin amorphous carbon film inspired by anti-reflection architecture in butterfly wing. Carbon, 2011. 49(3): p. 877-883.

181. Yang, Z.-P., L. Ci, J.A. Bur, S.-Y. Lin, and P.M. Ajayan, Experimental observation of an extremely dark material made by a low-density nanotube array. Nano letters, 2008. 8(2): p. $446-451$.

182. Hu, X., W. Xu, L. Zhou, Y. Tan, Y. Wang, S. Zhu, and J. Zhu, Tailoring graphene oxide-based aerogels for efficient solar steam generation under one sun. Advanced materials, 2017. 29(5): p. 1604031.

183. Liu, Z., Z. Yang, X. Huang, C. Xuan, J. Xie, H. Fu, Q. Wu, J. Zhang, X. Zhou, and Y. Liu, High-absorption recyclable photothermal membranes used in a bionic system for high-efficiency solar desalination via enhanced localized heating. Journal of Materials Chemistry A, 2017. 5(37): p. 20044-20052.

184. Mizuno, K., J. Ishii, H. Kishida, Y. Hayamizu, S. Yasuda, D.N. Futaba, M. Yumura, and $\mathrm{K}$. Hata, A black body absorber from vertically aligned single-walled carbon nanotubes. Proceedings of the National Academy of Sciences, 2009. 106(15): p. 6044-6047.

185. Zhang, P., J. Li, L. Lv, Y. Zhao, and L. Qu, Vertically aligned graphene sheets membrane for highly efficient solar thermal generation of clean water. Acs Nano, 2017. 11(5): p. 5087-5093.

186. Zhao, F., X. Zhou, Y. Shi, X. Qian, M. Alexander, X. Zhao, S. Mendez, R. Yang, L. Qu, and G. Yu, Highly efficient solar vapour generation via hierarchically nanostructured gels. Nature nanotechnology, 2018. 13(6): p. 489.

187. Liu, M., X. Yin, E. Ulin-Avila, B. Geng, T. Zentgraf, L. Ju, F. Wang, and X. Zhang, A graphene-based broadband optical modulator. Nature, 2011. 474(7349): p. 64-67.

188. Li, W., Z. Li, K. Bertelsmann, and D.E. Fan, Portable Low-Pressure Solar SteamingCollection Unisystem with Polypyrrole Origamis. Advanced Materials, 2019. 31(29): p. 1900720. 
189. Wang, X., Q. Liu, S. Wu, B. Xu, and H. Xu, Multilayer Polypyrrole Nanosheets with Self-Organized Surface Structures for Flexible and Efficient Solar-Thermal Energy Conversion. Advanced Materials, 2019. 31(19): p. 1807716.

190. Masuda, H. and K. Fukuda, Ordered metal nanohole arrays made by a two-step replication of honeycomb structures of anodic alumina. science, 1995. 268(5216): p. 1466-1468.

191. Neumann, O., A.S. Urban, J. Day, S. Lal, P. Nordlander, and N.J. Halas, Solar vapor generation enabled by nanoparticles. ACS nano, 2013. 7(1): p. 42-49.

192. Jin, H., G. Lin, L. Bai, A. Zeiny, and D. Wen, Steam generation in a nanoparticle-based solar receiver. Nano Energy, 2016. 28: p. 397-406.

193. Prasher, R., P.E. Phelan, and P. Bhattacharya, Effect of aggregation kinetics on the thermal conductivity of nanoscale colloidal solutions (nanofluid). Nano letters, 2006. 6(7): p. 1529-1534.

194. Shi, L., Y. Wang, L. Zhang, and P. Wang, Rational design of a bi-layered reduced graphene oxide film on polystyrene foam for solar-driven interfacial water evaporation. Journal of Materials Chemistry A, 2017. 5(31): p. 16212-16219.

195. Yang, Y., R. Zhao, T. Zhang, K. Zhao, P. Xiao, Y. Ma, P.M. Ajayan, G. Shi, and Y. Chen, Graphene-based standalone solar energy converter for water desalination and purification. ACS nano, 2018. 12(1): p. 829-835.

196. Xue, G., K. Liu, Q. Chen, P. Yang, J. Li, T. Ding, J. Duan, B. Qi, and J. Zhou, Robust and low-cost flame-treated wood for high-performance solar steam generation. ACS applied materials \& interfaces, 2017. 9(17): p. 15052-15057.

197. Liu, P.-F., L. Miao, Z. Deng, J. Zhou, H. Su, L. Sun, S. Tanemura, W. Cao, F. Jiang, and L.-D. Zhao, A mimetic transpiration system for record high conversion efficiency in solar steam generator under one-sun. Materials today energy, 2018. 8: p. 166-173.

198. Guo, Y., X. Zhou, F. Zhao, J. Bae, B. Rosenberger, and G. Yu, Synergistic energy nanoconfinement and water activation in hydrogels for efficient solar water desalination. ACS nano, 2019. 13(7): p. 7913-7919.

199. Zhou, X., F. Zhao, Y. Guo, B. Rosenberger, and G. Yu, Architecting highly hydratable polymer networks to tune the water state for solar water purification. Science advances, 2019. 5(6): p. eaaw5484. 
200. Abu-Arabi, M., M. Al-harahsheh, H. Mousa, and Z. Alzghoul, Theoretical investigation of solar desalination with solar still having phase change material and connected to a solar collector. Desalination, 2018. 448: p. 60-68.

201. Ebenstein, A., The consequences of industrialization: evidence from water pollution and digestive cancers in China. Review of Economics and Statistics, 2012. 94(1): p. 186-201.

202. Postel, S.L., G.C. Daily, and P.R. Ehrlich, Human appropriation of renewable fresh water. Science, 1996. 271(5250): p. 785-788.

203. Yuan, J., X. Liu, O. Akbulut, J. Hu, S.L. Suib, J. Kong, and F. Stellacci, Superwetting nanowire membranes for selective absorption. Nature Nanotechnology, 2008. 3(6): p. 332.

204. Xue, Z., S. Wang, L. Lin, L. Chen, M. Liu, L. Feng, and L. Jiang, A novel superhydrophilic and underwater superoleophobic hydrogel-coated mesh for oil/water separation. Advanced Materials, 2011. 23(37): p. 4270-4273.

205. Zhang, J. and S. Seeger, Polyester materials with superwetting silicone nanofilaments for oil/water separation and selective oil absorption. Advanced Functional Materials, 2011. 21(24): p. 4699-4704.

206. Jin, M., J. Wang, X. Yao, M. Liao, Y. Zhao, and L. Jiang, Underwater Oil Capture by a Three-Dimensional Network Architectured Organosilane Surface. Advanced Materials, 2011. 23(25): p. 2861-2864.

207. Liu, K., X. Yao, and L. Jiang, Recent developments in bio-inspired special wettability. Chemical Society Reviews, 2010. 39(8): p. 3240-3255.

208. Chou, J.B., Y.X. Yeng, Y.E. Lee, A. Lenert, V. Rinnerbauer, I. Celanovic, M. Soljačić, N.X. Fang, E.N. Wang, and S.G. Kim, Enabling ideal selective solar absorption with $2 D$ metallic dielectric photonic crystals. Advanced Materials, 2014. 26(47): p. 8041-8045.

209. Zhu, Y., F. Zhang, D. Wang, X.F. Pei, W. Zhang, and J. Jin, A novel zwitterionic polyelectrolyte grafted PVDF membrane for thoroughly separating oil from water with ultrahigh efficiency. Journal of Materials Chemistry A, 2013. 1(18): p. 5758-5765.

210. Meng, X., M. Wang, L. Heng, and L. Jiang, Underwater Mechanically Robust OilRepellent Materials: Combining Conflicting Properties Using a Heterostructure. Advanced Materials, 2018. 
211. Gu, Y., J. Yang, and S. Zhou, A facile immersion-curing approach to surface-tailored poly (vinyl alcohol)/silica underwater superoleophobic coatings with improved transparency and robustness. Journal of Materials Chemistry A, 2017. 5(22): p. 1086610875.

212. Chen, F., Y. Lu, X. Liu, J. Song, G. He, M.K. Tiwari, C.J. Carmalt, and I.P. Parkin, Table salt as a template to prepare reusable porous PVDF-MWCNT foam for separation of immiscible oils/organic solvents and corrosive aqueous solutions. Advanced Functional Materials, 2017. 27(41).

213. Zhu, Y., W. Xie, F. Zhang, T. Xing, and J. Jin, Superhydrophilic in-situ-cross-linked zwitterionic polyelectrolyte/PVDF-blend membrane for highly efficient oil/water emulsion separation. ACS applied materials \& interfaces, 2017. 9(11): p. 9603-9613.

214. Ge, J., D. Zong, Q. Jin, J. Yu, and B. Ding, Biomimetic and superwettable nanofibrous skins for highly efficient separation of oil-in-water emulsions. Advanced Functional Materials, 2018. 28(10): p. 1705051.

215. Xu, N., J. Li, Y. Wang, C. Fang, X. Li, Y. Wang, L. Zhou, B. Zhu, Z. Wu, and S. Zhu, A water lily-inspired hierarchical design for stable and efficient solar evaporation of highsalinity brine. Science advances, 2019. 5(7): p. eaaw7013.

216. Qiu, P., F. Liu, C. Xu, H. Chen, F. Jiang, Y. Li, and Z. Guo, Porous three-dimensional carbon foams with interconnected microchannels for high-efficiency solar-to-vapor conversion and desalination. Journal of Materials Chemistry A, 2019. 7(21): p. 1303613042.

217. Gong, F.F., H. Li, W. Wang, J. Huang, D.D. Xia, J. Liao, M. Wu, and D.V. Papavassiliou, Scalable, eco-friendly and ultrafast solar steam generators based on one-step melaminederived carbon sponges toward water purification. Nano Energy, 2019. 58: p. 322-330.

218. Zhang, Z., P. Mu, J. Han, J. He, Z. Zhu, H. Sun, W. Liang, and A. Li, Superwetting and mechanically robust $\mathrm{MnO} 2$ nanowire-reduced graphene oxide monolithic aerogels for efficient solar vapor generation. Journal of Materials Chemistry A, 2019. 7(30): p. 18092-18099.

219. Xu, W., X. Yun, J. Liu, H. Wu, Y. Cui, D. Li, D. Guo, C. Li, A. Liu, and H. Bai, Efficient Water Transport and Solar Steam Generation via Radially, Hierarchically Structured Aerogels. ACS nano, 2019. 
220. Hu, R., J. Zhang, Y. Kuang, K. Wang, X. Cai, Z. Fang, W. Huang, G. Chen, and Z. Wang, A Janus evaporator with low tortuosity for long-term solar desalination. Journal of Materials Chemistry A, 2019. 7(25): p. 15333-15340.

221. Wu, X., M.E. Robson, J.L. Phelps, J.S. Tan, B. Shao, G. Owens, and H. Xu, A flexible photothermal cotton-CuS nanocage-agarose aerogel towards portable solar steam generation. Nano energy, 2019. 56: p. 708-715.

222. Zhang, S., G. Jiang, S. Gao, H. Jin, Y. Zhu, F. Zhang, and J. Jin, Cupric phosphate nanosheets-wrapped inorganic membranes with superhydrophilic and outstanding anticrude oil-fouling property for oil/water separation. ACS nano, 2018. 12(1): p. 795803.

223. Wen, G. and Z. Guo, Nonflammable superhydrophobic paper with biomimetic layered structure exhibiting boiling-water resistance and repairable properties for emulsion separation. Journal of Materials Chemistry A, 2018. 6(16): p. 7042-7052.

224. Zhang, L., L. Cheng, H. Wu, T. Yoshioka, and H. Matsuyama, One-step fabrication of robust and anti-oil-fouling aliphatic polyketone composite membranes for sustainable and efficient filtration of oil-in-water emulsions. Journal of Materials Chemistry A, 2018. 6(47): p. 24641-24650.

225. Zhang, W., X. Li, R. Qu, Y. Liu, Y. Wei, and L. Feng, Janus membrane decorated via a versatile immersion-spray route: controllable stabilized oil/water emulsion separation satisfying industrial emission and purification criteria. Journal of Materials Chemistry A, 2019. 7(9): p. 4941-4949.

226. Zhang, L., Y. Lin, H. Wu, L. Cheng, Y. Sun, T. Yasui, Z. Yang, S. Wang, T. Yoshioka, and H. Matsuyama, An ultrathin in situ silicification layer developed by an electrostatic attraction force strategy for ultrahigh-performance oil-water emulsion separation. Journal of Materials Chemistry A, 2019. 7(42): p. 24569-24582.

227. Oh, S., S. Ki, S. Ryu, M.C. Shin, J. Lee, C. Lee, and Y. Nam, Performance Analysis of Gravity-Driven Oil-Water Separation Using Membranes with Special Wettability. Langmuir, 2019.

228. Dong, X., S. Gao, J. Huang, S. Li, T. Zhu, Y. Cheng, Y. Zhao, Z. Chen, and Y. Lai, A self-roughened and biodegradable superhydrophobic coating with UV shielding, solar- 
induced self-healing and versatile oil-water separation ability. Journal of Materials Chemistry A, 2019. 7(5): p. 2122-2128.

229. Alhosseini, S.N., F. Moztarzadeh, M. Mozafari, S. Asgari, M. Dodel, A. Samadikuchaksaraei, S. Kargozar, and N. Jalali, Synthesis and characterization of electrospun polyvinyl alcohol nanofibrous scaffolds modified by blending with chitosan for neural tissue engineering. International journal of nanomedicine, 2012. 7: p. 25.

230. López-García, F., G. Canché-Escamilla, A. Ocampo-Flores, P. Roquero-Tejeda, and L. Ordóñez, Controlled size nano-polypyrrole synthetized in micro-emulsions as PT support for the ethanol electro-oxidation reaction. Int. J. Electrochem. Sci, 2013. 8: p. 3794-3813.

231. Liu, Y., Y. Chu, and L. Yang, Adjusting the inner-structure of polypyrrole nanoparticles through microemulsion polymerization. Materials chemistry and physics, 2006. 98(2-3): p. 304-308.

232. Gao, S., Y. Zhu, J. Wang, F. Zhang, J. Li, and J. Jin, Layer-by-Layer Construction of Cu2+/Alginate Multilayer Modified Ultrafiltration Membrane with Bioinspired Superwetting Property for High-Efficient Crude-Oil-in-Water Emulsion Separation. Advanced Functional Materials, 2018. 28(49): p. 1801944.

233. Meng, F.-N., M.-Q. Zhang, K. Ding, T. Zhang, and Y.-K. Gong, Cell membrane mimetic PVDF microfiltration membrane with enhanced antifouling and separation performance for oil/water mixtures. Journal of Materials Chemistry A, 2018. 6(7): p. 3231-3241.

234. Vargaftik, N., B. Volkov, and L. Voljak, International tables of the surface tension of water. Journal of Physical and Chemical Reference Data, 1983. 12(3): p. 817-820.

235. Ghosh, P., Shape of the interface. NPTEL-Chemical Engineering-Interfacial Engineering, IIT Guwahati, Guwahati India, https://nptel/. ac. in/courses/103103033/module2/lecture3. pdf, 2012.

236. Zhang, L., B. Tang, J. Wu, R. Li, and P. Wang, Hydrophobic Light-to-Heat Conversion Membranes with Self-Healing Ability for Interfacial Solar Heating. Advanced Materials, 2015. 27(33): p. 4889-4894.

237. Boyer, T.P., J.I. Antonov, O.K. Baranova, C. Coleman, H.E. Garcia, A. Grodsky, D.R. Johnson, R.A. Locarnini, A.V. Mishonov, and T.D. O'Brien, World Ocean Database 2013. 2013. 
238. Nandakumar, D.K., S.K. Ravi, Y. Zhang, N. Guo, C. Zhang, and S.C. Tan, A super hygroscopic hydrogel for harnessing ambient humidity for energy conservation and harvesting. Energy \& Environmental Science, 2018. 11(8): p. 2179-2187.

239. Nandakumar, D.K., Y. Zhang, S.K. Ravi, N. Guo, C. Zhang, and S.C. Tan, Solar Energy Triggered Clean Water Harvesting from Humid Air Existing above Sea Surface Enabled by a Hydrogel with Ultrahigh Hygroscopicity. Advanced Materials, 2019. 31(10): p. 1806730.

240. Kresge, C.T. and W.J. Roth, The discovery of mesoporous molecular sieves from the twenty year perspective. Chemical Society Reviews, 2013. 42(9): p. 3663-3670.

241. Yu, L., K. Dean, and L. Li, Polymer blends and composites from renewable resources. Progress in polymer science, 2006. 31(6): p. 576-602.

242. Yang, L., D.K. Nandakumar, L. Miao, L. Suresh, D. Zhang, T. Xiong, J.V. Vaghasiya, K.C. Kwon, and S.C. Tan, Energy harvesting from atmospheric humidity by a hydrogelintegrated ferroelectric-semiconductor system. Joule, 2020. 4(1): p. 176-188.

243. Zhang, Y., L. Wu, X. Wang, J. Yu, and B. Ding, Super hygroscopic nanofibrous membrane-based moisture pump for solar-driven indoor dehumidification. Nature Communications, 2020. 11(1): p. 1-11.

244. Thickett, S.C., C. Neto, and A.T. Harris, Biomimetic surface coatings for atmospheric water capture prepared by dewetting of polymer films. Advanced Materials, 2011. 23(32): p. $3718-3722$.

245. Zhao, F., X. Zhou, Y. Liu, Y. Shi, Y. Dai, and G. Yu, Super Moisture-Absorbent Gels for All-Weather Atmospheric Water Harvesting. Advanced Materials, 2019. 31(10): p. 1806446.

246. Kim, H., S. Yang, S.R. Rao, S. Narayanan, E.A. Kapustin, H. Furukawa, A.S. Umans, O.M. Yaghi, and E.N. Wang, Water harvesting from air with metal-organic frameworks powered by natural sunlight. Science, 2017. 356(6336): p. 430-434.

247. Ju, J., H. Bai, Y. Zheng, T. Zhao, R. Fang, and L. Jiang, A multi-structural and multifunctional integrated fog collection system in cactus. Nature communications, 2012. 3(1): p. 1-6.

248. Kaseke, K.F., L. Wang, and M.K. Seely, Nonrainfall water origins and formation mechanisms. Science Advances, 2017. 3(3): p. e1603131. 
249. Wu, J., Y. Wu, F. Yang, C. Tang, Q. Huang, and J. Zhang, Impact of delignification on morphological, optical and mechanical properties of transparent wood. Composites Part A: Applied Science and Manufacturing, 2019. 117: p. 324-331.

250. Li, Y., M. Cheng, E. Jungstedt, B. Xu, L. Sun, and L. Berglund, Optically transparent wood substrate for perovskite solar cells. ACS sustainable chemistry \& engineering, 2019. 7(6): p. 6061-6067.

251. Zhu, M., J. Song, T. Li, A. Gong, Y. Wang, J. Dai, Y. Yao, W. Luo, D. Henderson, and L. Hu, Highly anisotropic, highly transparent wood composites. Advanced materials, 2016. 28(26): p. 5181-5187.

252. Fu, K.K., Z. Wang, J. Dai, M. Carter, and L. Hu, Transient electronics: materials and devices. Chemistry of Materials, 2016. 28(11): p. 3527-3539.

253. Song, J., C. Chen, C. Wang, Y. Kuang, Y. Li, F. Jiang, Y. Li, E. Hitz, Y. Zhang, and B. Liu, Superflexible wood. ACS applied materials \& interfaces, 2017. 9(28): p. 2352023527.

254. Zhu, H., W. Luo, P.N. Ciesielski, Z. Fang, J. Zhu, G. Henriksson, M.E. Himmel, and L. $\mathrm{Hu}$, Wood-derived materials for green electronics, biological devices, and energy applications. Chemical Reviews, 2016. 116(16): p. 9305-9374.

255. Guan, H., Z. Cheng, and X. Wang, Highly compressible wood sponges with a spring-like lamellar structure as effective and reusable oil absorbents. ACS nano, 2018. 12(10): p. 10365-10373.

256. Wu, Y., J. Zhou, Q. Huang, F. Yang, Y. Wang, X. Liang, and J. Li, Study on the colorimetry properties of transparent wood prepared from six wood species. ACS omega, 2020. 5(4): p. 1782-1788.

257. Chen, L., Z. Xu, F. Wang, G. Duan, W. Xu, G. Zhang, H. Yang, J. Liu, and S. Jiang, $A$ flame-retardant and transparent wood/polyimide composite with excellent mechanical strength. Composites Communications, 2020.

258. Wu, Y., J. Zhou, Q. Huang, F. Yang, Y. Wang, and J. Wang, Study on the Properties of Partially Transparent Wood under Different Delignification Processes. Polymers, 2020. 12(3): p. 661.

259. Zhang, L., A. Wang, T. Zhu, Z. Chen, Y. Wu, and Y. Gao, Transparent Wood Composites Fabricated by Impregnation of Epoxy Resin and W-doped VO2 
Nanoparticles for Application in Energy-saving Windows. ACS Applied Materials \& Interfaces, 2020.

260. Mi, R., T. Li, D. Dalgo, C. Chen, Y. Kuang, S. He, X. Zhao, W. Xie, W. Gan, and J. Zhu, A Clear, Strong, and Thermally Insulated Transparent Wood for Energy Efficient Windows. Advanced Functional Materials, 2020. 30(1): p. 1907511.

261. Ma, Q., K. Pang, K. Wang, S. Huang, B. Ding, Y. Duan, and J. Zhang, Ultrafine and carboxylated $\beta$-chitin nanofibers prepared from squid pen and its transparent hydrogels. Carbohydrate polymers, 2019. 211: p. 118-123.

262. Kim, T., T.H. Tran, S.Y. Hwang, J. Park, D.X. Oh, and B.-S. Kim, Crab-on-a-tree: all biorenewable, optical and radio frequency transparent barrier nanocoating for food packaging. ACS nano, 2019. 13(4): p. 3796-3805.

263. Aranday-García, R., H. Saimoto, K. Shirai, and S. Ifuku, Chitin biological extraction from shrimp wastes and its fibrillation for elastic nanofiber sheets preparation. Carbohydrate polymers, 2019. 213: p. 112-120.

264. He, M., X. Wang, Z. Wang, L. Chen, Y. Lu, X. Zhang, M. Li, Z. Liu, Y. Zhang, and H. Xia, Biocompatible and biodegradable bioplastics constructed from chitin via a "green" pathway for bone repair. ACS Sustainable Chemistry \& Engineering, 2017. 5(10): p. 9126-9135.

265. Wijesena, R.N., N.D. Tissera, V. Rathnayaka, H. Rajapakse, R.M. de Silva, and K.N. de Silva, Shape-stabilization of polyethylene glycol phase change materials with chitin nanofibers for applications in "smart" windows. Carbohydrate Polymers, 2020: p. 116132.

266. Wu, Q., N.E. Mushi, and L.A. Berglund, High-Strength Nanostructured Films Based on Well-Preserved $\alpha$-Chitin Nanofibrils Disintegrated from Insect Cuticles. Biomacromolecules, 2019. 21(2): p. 604-612.

267. Zhu, K., S. Shi, Y. Cao, A. Lu, J. Hu, and L. Zhang, Robust chitin films with good biocompatibility and breathable properties. Carbohydrate polymers, 2019. 212: p. 361367.

268. Yihun, F.A., S. Ifuk, H. Saimoto, H. Izawa, and M. Morimoto, Highly transparent and flexible surface modified chitin nanofibers reinforced poly (methyl methacrylate) nanocomposites: Mechanical, thermal and optical studies. Polymer, 2020: p. 122497. 
269. Wijesena, R.N., N.D. Tissera, V. Rathnayaka, R.M. de Silva, and K.N. de Silva, Colloidal stability of chitin nanofibers in aqueous systems: Effect of $\mathrm{pH}$, ionic strength, temperature \& concentration. Carbohydrate Polymers, 2020. 235: p. 116024.

270. Nawawi, W.M.F.W., K.-Y. Lee, E. Kontturi, A. Bismarck, and A. Mautner, Surface properties of chitin-glucan nanopapers from Agaricus bisporus. International Journal of Biological Macromolecules, 2020. 148: p. 677-687.

271. Kadokawa, J.-i., S. Idenoue, and K. Yamamoto, Fabricating Chitin Paper from SelfAssembled Nanochitins. ACS Sustainable Chemistry \& Engineering, 2020.

272. Coltelli, M.-B., P. Cinelli, V. Gigante, L. Aliotta, P. Morganti, L. Panariello, and A. Lazzeri, Chitin nanofibrils in poly (Lactic acid)(PLA) nanocomposites: Dispersion and thermo-mechanical properties. International journal of molecular sciences, 2019. 20(3): p. 504.

273. Ifuku, S., S. Morooka, A.N. Nakagaito, M. Morimoto, and H. Saimoto, Preparation and characterization of optically transparent chitin nanofiber/(meth) acrylic resin composites. Green Chemistry, 2011. 13(7): p. 1708-1711. 


\section{Appendix}

\section{Hygroscopic Hydrogel for Ambient Moisture Scavenging}

\subsection{Abstract}

Ambient humidity as a potential new freshwater resource is utilized in this study. A hygroscopic hydrogel was synthesized with an aim of collecting ambient moisture. The hydrogel is composed of PVP and PPy, in which the superhydrophilicity enables the gel to absorb moisture from air. PNIPAM was incorporated in the gel for water releasing after the water absorption due to the thermos-responsive effect of PNIPAM.

\subsection{Introduction}

One of the universally present but largely overlooked water sources is ambient humidity. It is reported that the atmosphere contains 141 million billion liters of water, which is equivalent to approximately $10 \%$ of the freshwater from lakes and rivers[238]. A few attempts have demonstrated the possibility of scavenging water vapor from air by hygroscopic materials based on surface water absorption[239-243]. Such materials show prominent humidity scavenging capability but suffers from low-efficient water releasing[244], hindering their possibility as ambient water harvester. However, it has been widely found in animals and plants to harvest water from atmosphere for survival[245]. For instance, Agamid lizard, dune grass and cacti adopted the technique of using atmospheric moisture, which inspires biomimetic hygroscopic hydrogels for ambient humidity collection[246]. Until now, multiple artificial atmospheric water harvesters have been proposed, using hygroscopic materials such as molecular sieves, polymeric desiccants and silica gels.[247, 248] However, these materials exhibit strong interaction with 
water, which hinders the water releasing. A moisture scavenging system with the ability to absorb atmospheric water at low relative humidity $(\mathrm{RH})$ level and release liquid water is yet to be developed.

Herein, an ambient humid harvesting hydrogel that is capable of absorbing vapor from atmosphere and converting to collectable liquid water was synthesized. The hydrogel is composed of hygroscopic polyvinylpyrrolidone (PVP), PPy and temperature-responsive poly Nisopropylacrylamide (PNIPAM). The synergistic effect of this interpenetrating hydrogel network enables controllable water capturing and water releasing under various relative humidity. Furthermore, the functionality of the hydrogel network opens the possibility of water management with high-capacity storage, water transportation through polymeric network and controllable phase transformation. This material provides a possible solution to freshwater crisis through advanced atmospheric water harvesting.

\subsection{Experimental Section}

\subsubsection{Material and Chemicals}

PVP (100,000 $\left.\mathrm{g} \mathrm{mol}^{-1}\right)$, ammonium persulfate (APS), Agar, N, N'-methylenebisacrylamide (MBA) were purchased from Sigma Aldrich. Pyrrole was purchased from fisher scientific.

\subsubsection{Fabrications}

PVP was dissolved in DI water to prepare for $20 \mathrm{wt} \%$ PVP solution. $4 \mathrm{wt} \%$ Agar was prepared and heated at $95{ }^{\circ} \mathrm{C}$ for 30 minutes prior to use. APS was dissolved in water to prepare for $40 \mathrm{wt} \%$ solution. In a typical synthesis of PVP hydrogel, 12\% PVP, $1 \%$ agar and 10\% APS solution were mixed. The bubbles in the solution were removed by centrifugation for 5 minutes at a speed of $8000 \mathrm{rpm}$. The polymerization was carried out in water bath at $60{ }^{\circ} \mathrm{C}$ for 1 hour. To synthesize PVP-PNIPAM hydrogel, $1 \mathrm{ml}$ of $120 \mathrm{mg} / \mathrm{ml}$ NIPAM, $10 \mu \mathrm{lof} 10 \mathrm{mg} / \mathrm{ml}$ MBA, $10 \mu \mathrm{l}$ of 200 
$\mathrm{mg} / \mathrm{ml}$ APS were mixed with the solution used to prepare for PVP. The bubbles in the solution were removed by centrifugation for 5 minutes at a speed of $8000 \mathrm{rpm}$. The polymerization was carried out in water bath at $60{ }^{\circ} \mathrm{C}$ for 1 hour. The obtained hydrogel was immersed in cold water $\left(4{ }^{\circ} \mathrm{C}\right)$ and hot DI water $\left(80^{\circ} \mathrm{C}\right)$ alternately for 3 hours to remove unreacted monomers. The purification step was repeated 3 times.

The final step of the fabrication of hygroscopic hydrogel is coating the hydrogel with PPy. The hydrogel was dipped in a mixture of $\mathrm{Fe}^{3+}$ and pyrrole monomer solution. The slow oxidation of the pyrrole monomers was carried out at $4^{\circ} \mathrm{C}$ for 3 days. The reaction was stopped by washing the PPy coated hydrogel with running DI water for 5 minutes.

Characterization

\subsubsection{FTIR Test}

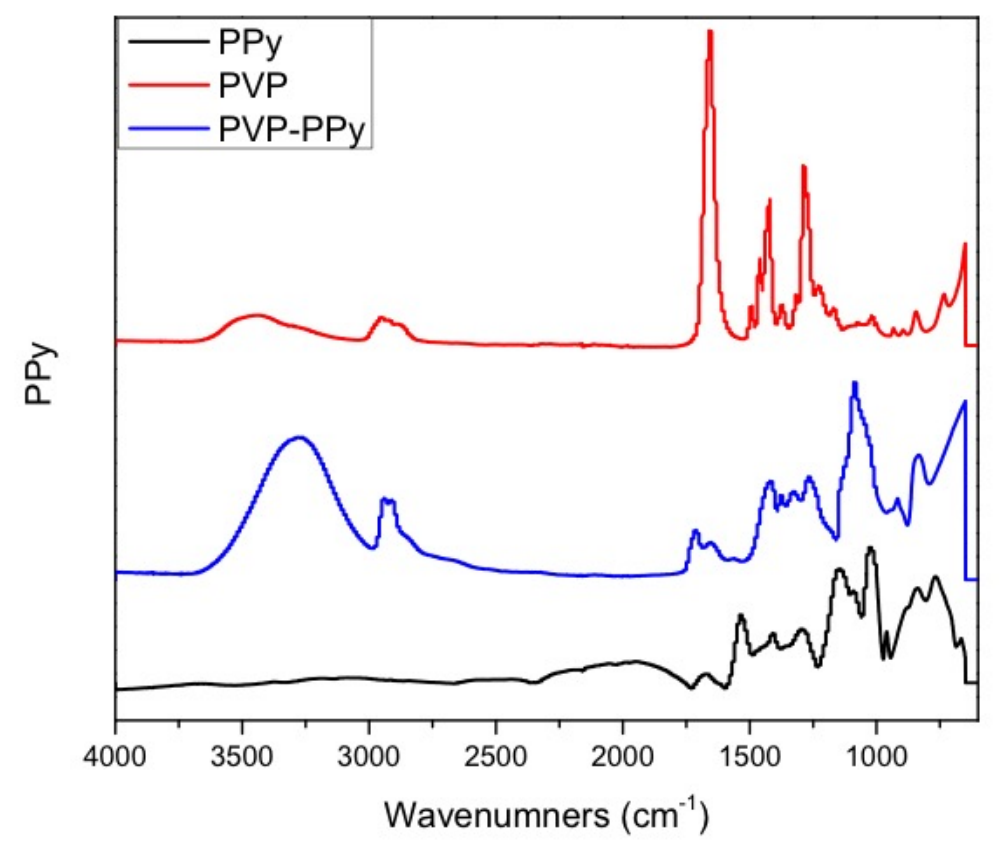

Figure A1-1. FTIR spectrum of PVP hydrogel (red), PPy particles (black) and PVP-PPy (blue). 
As can be observed from Figure A1-1, characteristic peaks for $-\mathrm{CH}$ groups of PVP at 2923 and $1460 \mathrm{~cm}^{-1}$ are present. The peak at $1290 \mathrm{~cm}^{-1}$ typical for $-\mathrm{C}-\mathrm{N}$ bond in PVP was also present in the FTIR spectrum. The characteristic peak observed at $1660 \mathrm{~cm}^{-1}$ indicates the presence of carbonyl group in PVP. The characteristic peak at $1290 \mathrm{~cm}^{-1}$ represents presence of $-\mathrm{C}-\mathrm{N}$ bond, which is present in both PVP sample and PVP-PPy sample. Peaks for $-\mathrm{CH}$ groups of PVP at 2923 and $1460 \mathrm{~cm}-1$ are present; the $-\mathrm{CH}$ peaks are present in PVP-PPy samples. Absorption peak 1315, which is the characteristic for $=\mathrm{C}-\mathrm{H}$ of PPy was observed in PVP-PPy samples; proves the presence of PPy in PVP-PPy sample. The presence of these characteristic peaks of PVP and PPy in the PVP-PPy sample prove the composition of PVP-PPy.

\subsubsection{Rheology}

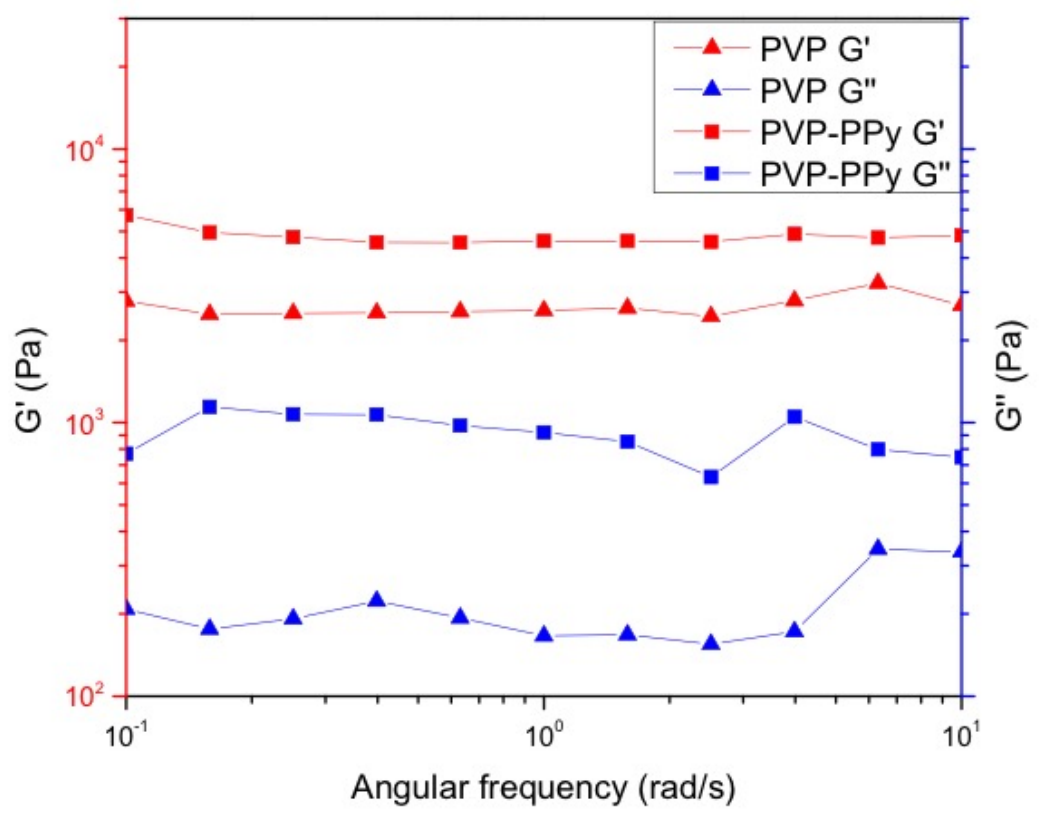

Figure A1-2. Rheology results of PVP hydrogel and PVP-PPy hydrogel.

The rheology test was performed at constant frequency of $1 \mathrm{~Hz}$. The elastic modulus and storage modulus of both pure PVP and PVP-PPy gel were tested. A wide linear viscoelastic region of 
PVP and PVP-PPy gels were observed in the dynamic frequency sweep experiments. The elastic modulus of both PVP and PVP-PPy gels are larger than their storage modulus. This result confirms the polymer skeleton of each gel. The higher elastic modulus of PVP-PPy suggests that the gel is more densely crosslinked than the PVP gel. The higher storage modulus of the PVPPPy shows that the polymer chains are restricted from slippage. The above results show that PPy molecules were incorporated in the network of PVP molecular mesh.

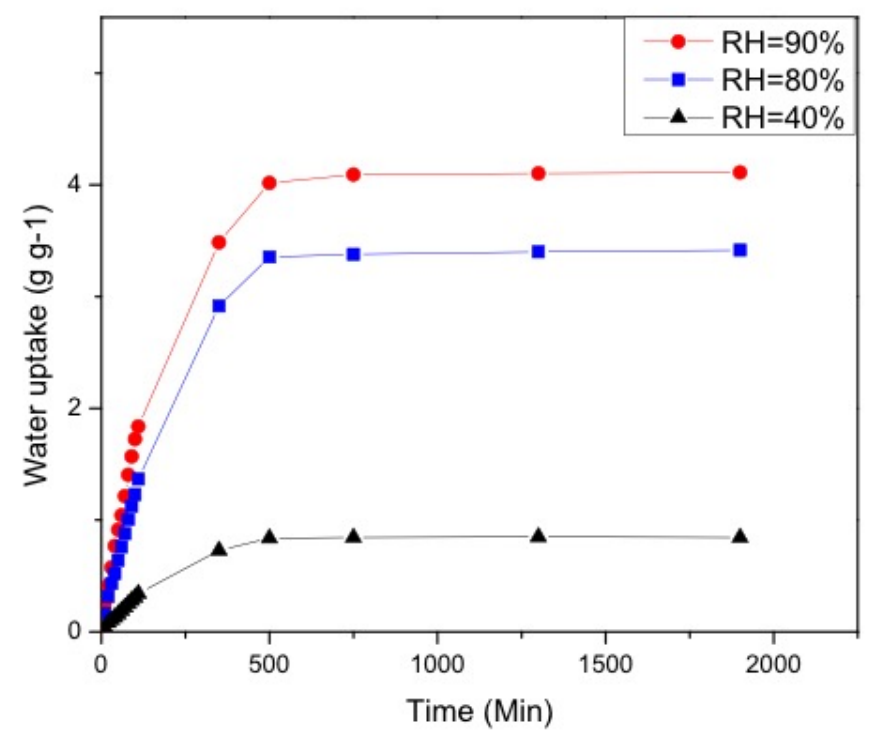

Figure A1-3. Water intake results of PVP-PPy gel under different humidity levels.

PVP-PPy gels were placed at three humidity environment (RH), $40 \%, 80 \%$ and $90 \%$, respectively. It took approximately 500 minutes, 530 minutes and 700 minutes for sufficient hydration of by absorbing water from air at humidity level of $90 \%, 80 \%$ and $40 \%$. The amount of absorbed water (when the hourly absorption increases lower than 1\%) are 4.2, 3.4 and $0.9 \mathrm{~g} \mathrm{~g}^{-}$ 1 of the corresponding dried PVP-PPy samples.

\subsubsection{Thermo-responsive Behavior}


PVP, PVP-PNIPAM and PNIPAM gels were prepared to compare the thermal-responsive behavior of the three gels. To achieve the water oozing goal, the ratio of PNIPAM and PVP were tuned. Pure PVP, 2.5 mol\% PVP in PNIPAM and 5 mol\% PVP in PNIPAM and pure PNIPAM were synthesized.

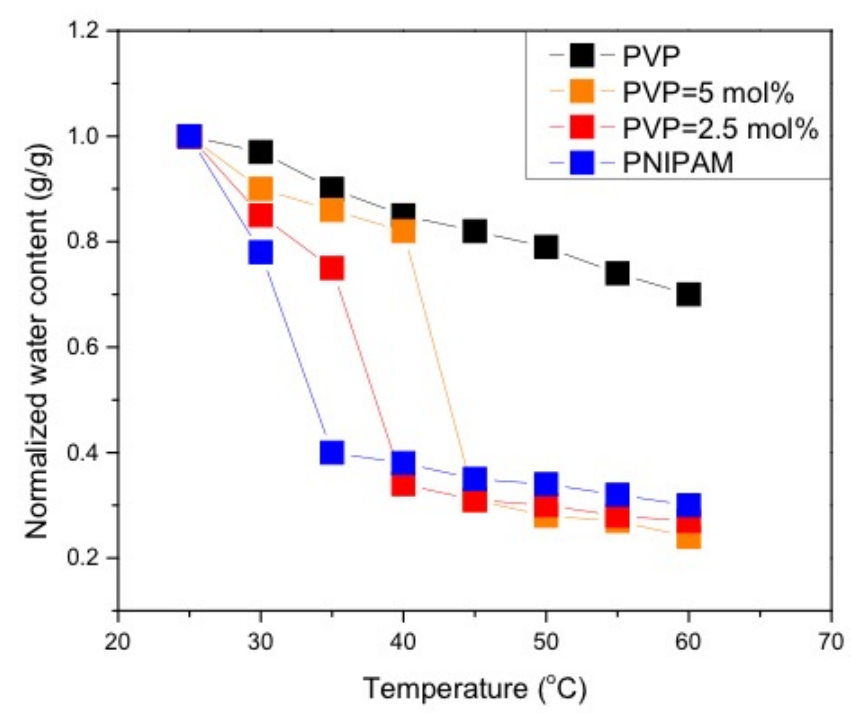

Figure A1-4. Water releasing performances of pure PVP, pure PNIPAM and PVP with different content of PNIPAM.

The lower critical solution temperature (LCST) of the PVP/PNIPAM is increased with higher molar concentration of PVP. The pure PVP shows no significant low critical solution temperature. The PNIPAM shows an LCST of $32{ }^{\circ} \mathrm{C} .2 .5 \mathrm{~mol} \%$ and $5 \mathrm{~mol} \%$ PVP-PNIPAM have higher LCST than pure PNIPAM. It is also observed that the shrieked PVP or PVP/PNIPAM has higher water content in the get, which indicate that the introduction of PNIPAM enhanced the moisture absorption capacity of the hydrogel but also increased the LCST. 


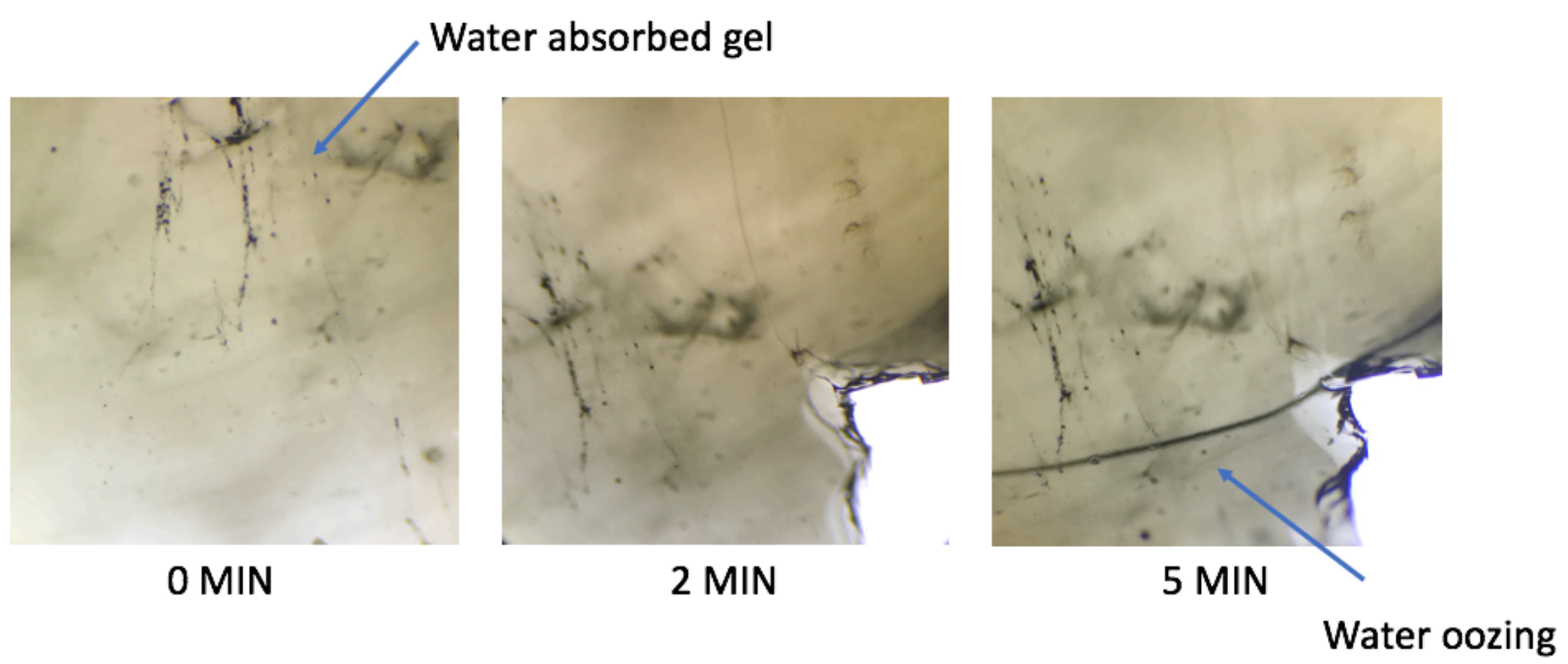

Figure A1-5. Water oozing from 2.5 mol\% PVP/PNIPAM gel beyond LCST.

With microscopic image under $\mathrm{x} 4$ magnification, the water oozing behavior was captured under heating of $50{ }^{\circ} \mathrm{C}$. Initially, the PVP/PNIPAM gel was saturated with water as shown in image at 0 min. Visible water was observed on the surface of the gel when heating to $50{ }^{\circ} \mathrm{C}$. The water absorption continues to increase as the heating proceeds. This phenomenon indicates that thermos-responsive gel can be used to collect water previously absorbed.

\subsubsection{PVP with Various Crosslinking Densities}

The hydrogels of various crosslinking densities were prepared to compare the water absorbing abilities. The water absorbing performance tests were carried out at $80 \% \mathrm{RH}$.

\begin{tabular}{|l|l|l|l|l|}
\hline Sample & PVP & $\begin{array}{l}\text { Initiator } \\
\text { concentration }\end{array}$ & Crosslinking time & Water absorbed \\
\hline PVP-30 & $20 \%$ & $30 \%$ & $40 \mathrm{~min}$ & 0.372 \\
\hline PVP-40 & $20 \%$ & $40 \%$ & $40 \mathrm{~min}$ & 0.693 \\
\hline PVP-50 & $20 \%$ & $50 \%$ & $40 \mathrm{~min}$ & 1.073 \\
\hline
\end{tabular}

Table A1-1. Different crosslinking densities were achieved by changing APS concentrations. 


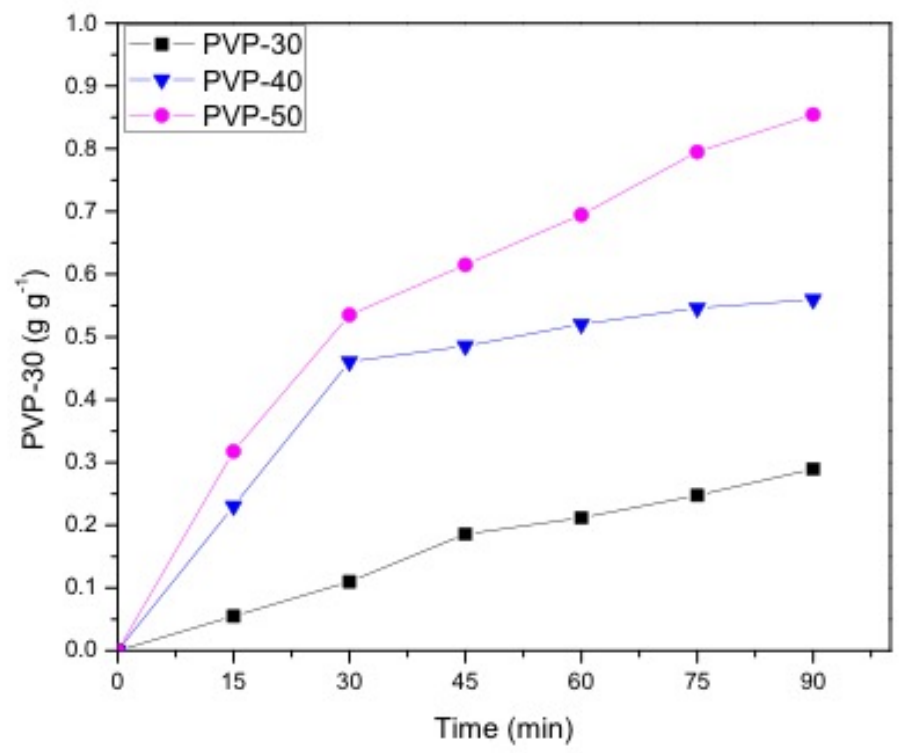

Figure A1-6. Water uptake performance of PVP gels with different crosslinking densities.

The initial weight for PVP-30, PVP-40 and PVP-50 are 1.285, 1.238 and 1.256g, respectively.

Figure A1-6 illustrates that the increased crosslinking density (from $30 \%$ to $50 \%$ of APS), increases the amount of water absorbed from the hydrogels. Water absorbing capacities for PVP30, PVP-40 and PVP-50 are $0.289 \mathrm{~g} \mathrm{~g}^{-1}, 0.559$ and $0.854 \mathrm{~g} \mathrm{~g}^{-1}$, respectively after 90 minutes under $80 \%$ RH. 


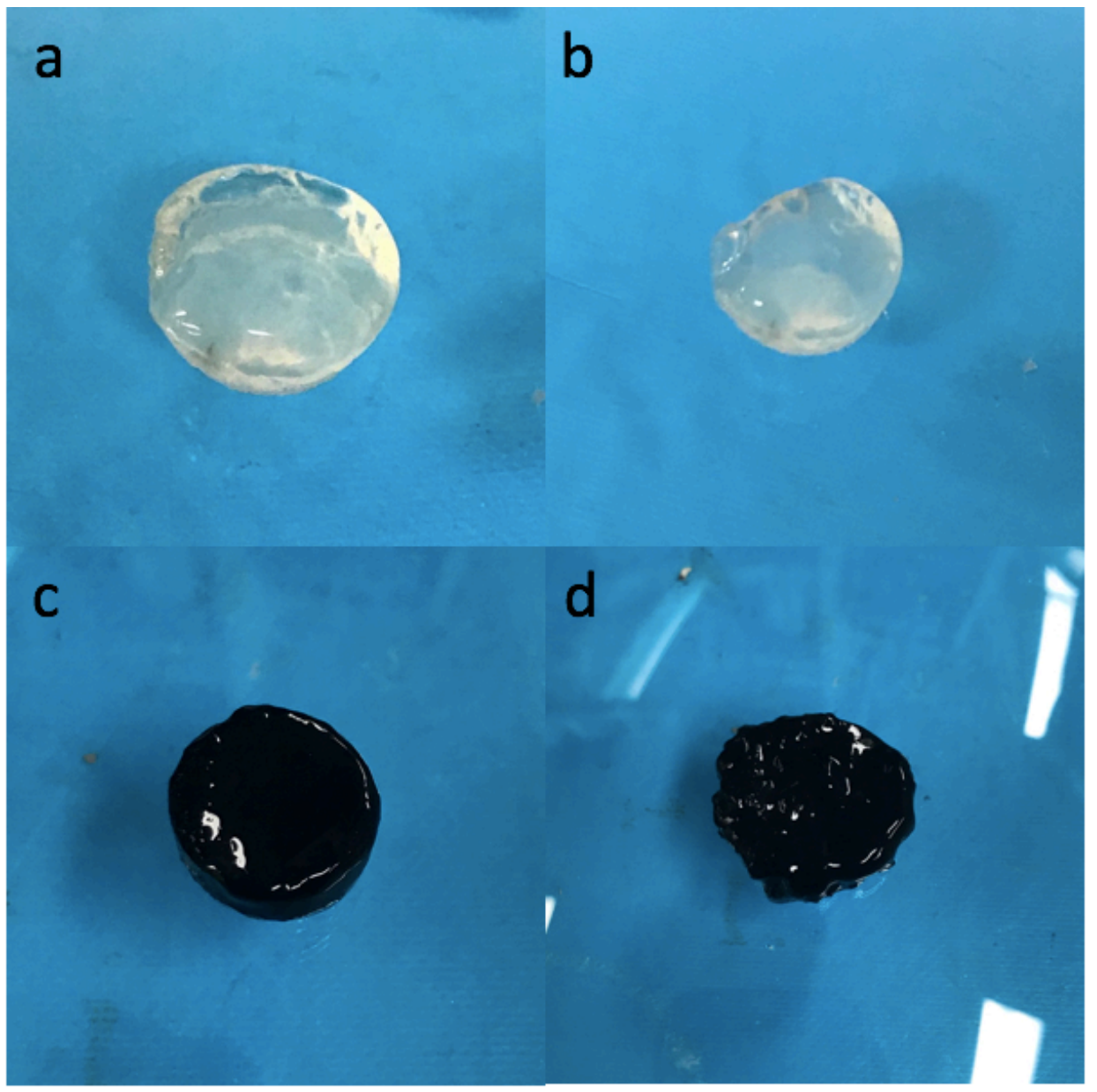

Figure A1-7. Images of PVP, PVP/PNIPAM, PVP-PPy and PVP/PNIPAM-PPy hydrogels.

\subsection{Conclusion}

In summary, PVP-PPy gel was prepared for scavenging humidity in atmosphere. FTIR and Rheology tests were carried out to characterize the hydrogel. PNIPAM was incorporated in the hydrogel to achieve thermal-responsive property. Three initiator concentrations were tested to study the impact of crosslinking densities on the water absorption ability of the hygroscopic gel. When the crosslinking densities increase, the water absorption capacities also increase. 


\section{Optically Transparent Wood-derived Glass}

\subsection{Abstract}

Wood polymer composites have been applied as structural materials in smart buildings, biomedical devices, electronics.[249, 250] The transparent wood composite shows potential in the above described applications as well as protection shield due to its low cost and high strength. In this study, Bulk Balsa wood was utilized as raw material to fabricate transparent woodPMMA composite.

\subsection{Introduction}

Optically transparent cellulose-based composites have attracted much attention as plausible material for structural material and electronic devices.[251] Wood as one of the most common resource of the cellulose-based composite shows multi-hierarchical structure shows strong mechanical properties.[252] Its anisotropic feature can be used for water transportation.[253] However, the opaque nature of wood makes the application in devices requiring transparency in materials nonviable.[254, 255] In this regard, optically transparent wood is an excellent asset for transparent structural materials and electronics application.

Pulp fibres as a source of cellulose nanofibers have been used in transparent films and paper manufacturing.[256] The main components of the pulp fibres are amorphous hemicellulose and cellulous fibres.[257] The major steps of transparent paper making is cellulose dissolution and regeneration and polymer impregnation.[258] However, the final products lack the mechanical strength of untreated wood with regard to their oriented cell structure and hierarchical construction, and this makes application in complex structures nonviable.[259] 
Transparent wood was first manufactured for preserved structure and it was later prepared for engineering applications.[260] Besides, several applications of transparent wood in structural optics and photonics and smart buildings were made. The anisotropic structure of wood endows the transparent wood composite with anisotropic property. Here in this project, the lignin in Balsa wood was removed and the deligninfied wood was filled with index matching polymers to achieve transparency. The original channels in wood samples are aligned with the wood plane. Different lignin removal processes and filling polymers were tested to improve transparency of the wood composites.

\subsection{Experimental Section}

\subsubsection{Material and Chemicals}

Balsa wood was air dried prior to chemical reaction. Sodium hydroxide (Fishers), hydrogen peroxide (35\% solution) and sodium sulphite (Sigma Aldrich). PMMA and PS were used as filtration polymers. Ethanol alcohol and deionized water were used for washing the wood samples.

\subsubsection{Delignification Treatment}

The chemical solution used for lignin removal was comprised of $\mathrm{Na}_{2} \mathrm{SO}_{3}\left(0.4 \mathrm{~mol} \mathrm{~L}^{-1}\right)$ and $\mathrm{NaOH}\left(2.5 \mathrm{~mol} \mathrm{~L}^{-1}\right)$ and heated to $90{ }^{\circ} \mathrm{C}$. Wood samples were prepared by cutting wood laminate with a thickness of $1 \mathrm{~mm}$ in the dimension of $20 \mathrm{~mm} * 20 \mathrm{~mm}$. The wood samples were immersed in delignification solution for 48 hours. After delignification, the wood samples were bleached with $\mathrm{H}_{2} \mathrm{O}_{2}$ solution at $100{ }^{\circ} \mathrm{C}$ without stirring until the samples turned white after the bleaching step. The reaction was stopped by washing and rinsing the samples with distilled water, followed by air drying.

\subsubsection{Polymer Infiltration}


5ml methyl methacrylate (MMA) monomer with 0.3 wt \% the initiator 2,2 - Azobis (2methylpropionitrile) (AIBN) was pre-polymerized at $70{ }^{\circ} \mathrm{C}$ for 20 minutes. The wood sample was infiltrated with the pre-polymerized MMA solution under vacuum for 40 minutes. The filtration was repeated for 3 times to ensure the polymer fully penetrated the wood sample. Lastly, the infiltrated wood was completed by heating the infiltrated wood sample at $70{ }^{\circ} \mathrm{C}$ for 4 hours. The fully polymerized wood composite was peeled off from the dish.

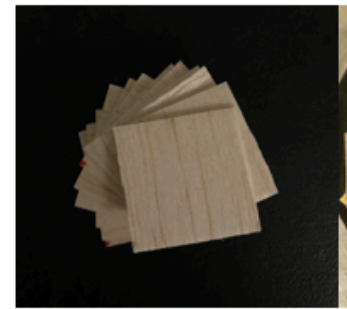

Original

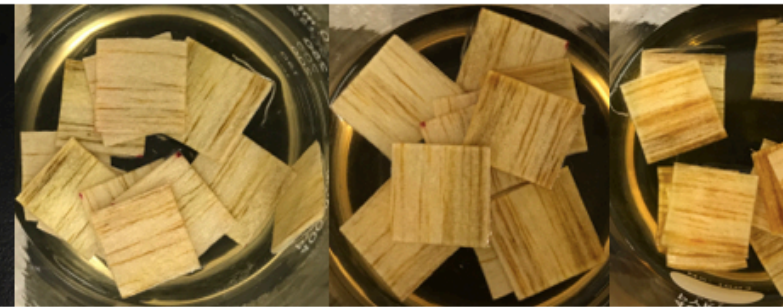

$1 \mathrm{H}$

$3 \mathrm{H}$

$7 \mathrm{H}$
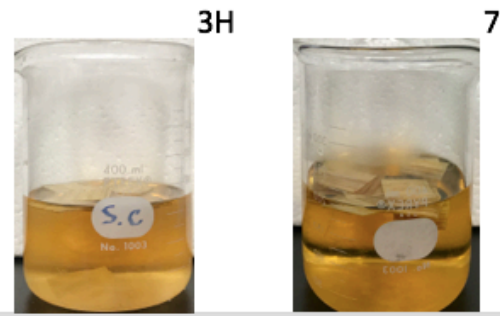

$14 \mathrm{H}$

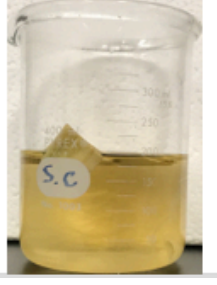

Figure A2-1. Lignin removal process of the wood samples.
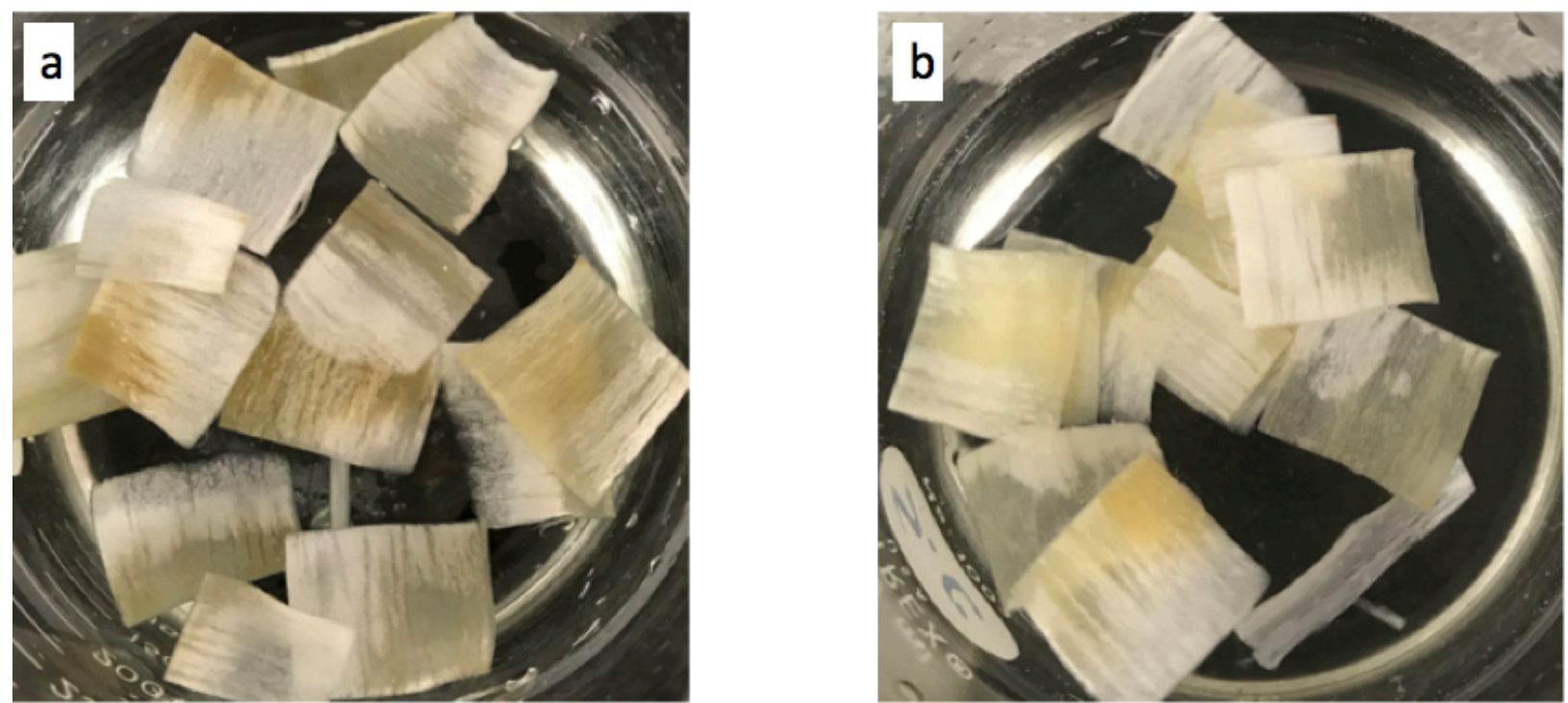

Figure A2-2. Wood samples immersed in $\mathrm{H}_{2} \mathrm{O}_{2}$ solution for 8 hours (left) and 12 hours (right). 
In the experiment, chemicals of conventional paper pulping procedure were used in this experiment. Sodium hydroxide and sodium sulphite solution was heated to $100{ }^{\circ} \mathrm{C}$ to remove the lignin in the wood. As can be seen in Figure A2-1, the colour of the solution became darker as the lignin removal process proceeds. The wood blocks were further delignified in $\mathrm{H}_{2} \mathrm{O}_{2}$ solution to eliminate the residual lignin in the sample (Figure A2-2). PMMA was infiltrated in the channel of the wood sample with repeated vacuum and devacuum process (Figure A2-3).
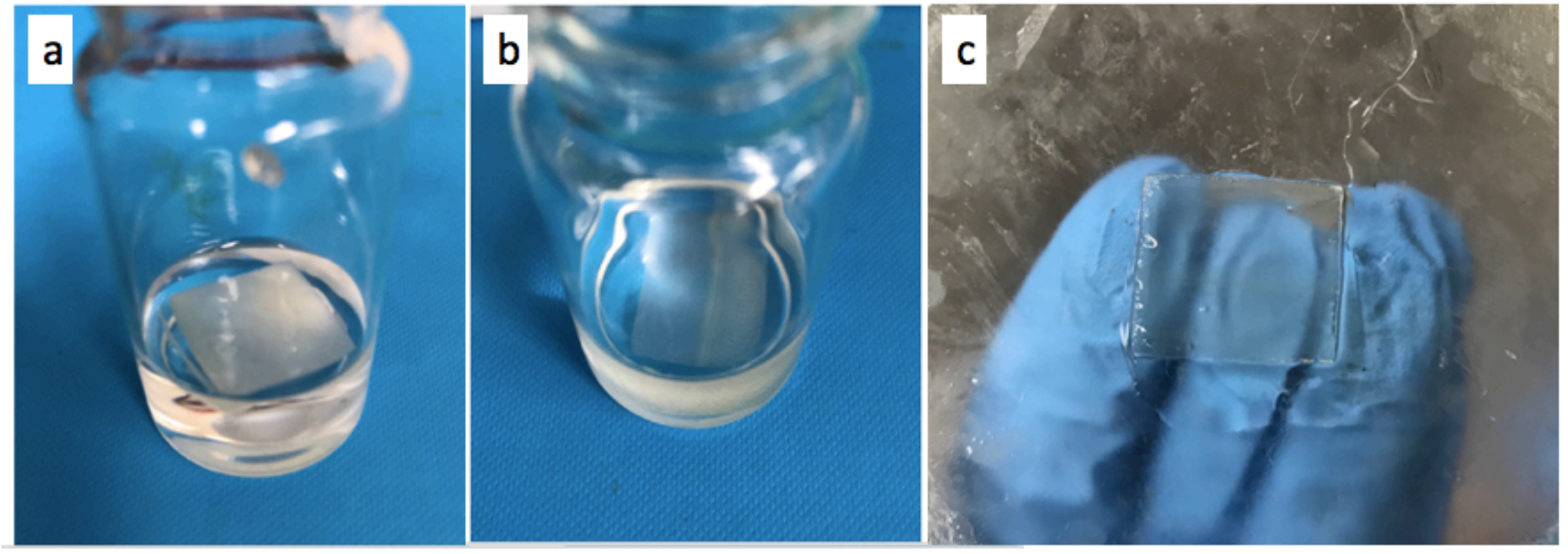

Figure A2-3. Wood sample immediately after immersed in MMA monomer (a), after vacuum and devacuum cycles for 3 times (b), and after polymerization of PMMA (c).

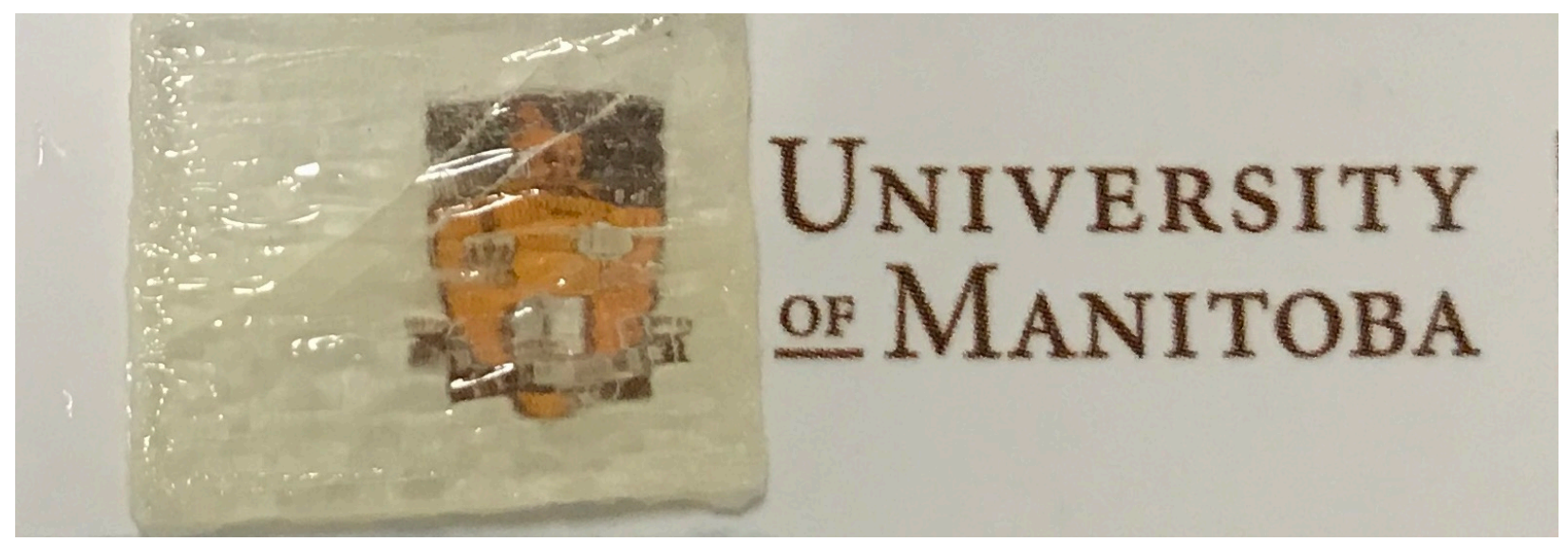

Figure A2-4. PMMA infiltrated wood sample on paper. 


\subsection{Conclusion}

Utilizing the unique structural nature of bulk wood, transparent wood-PMMA composite was fabricated in this study. The fabrication follows two mains steps: 1 . Removing the lignin in the channel of the wood laminate. And 2. filling the deligninfied wood sample with optically transparent polymer, in this case, PMMA. After the first step, white opaque wood sample with preserved nanostructure is obtained. Mechanical properties and optical properties can be studied in the future work, as well as potential applications. 


\section{Transparent Arthropods-derived Glass}

\subsection{Abstract}

Transparent chitin derived biopolymers hold the advantages of low toxicity, flexibility and toughness, which makes them potential alternatives to metallic incorporated or halogenated polymer films.[261, 262] In this study, transparent bioplastic with integrate arthropods exoskeleton was fabricated. The novel transparent shell biocomposite shows possible application as exterior for bio-mimetic robots.

\subsection{Introduction}

Chitin is the second most abundant biopolymers in nature.[263, 264] Chitin is produced from shellfish, fungi and insects.[265] Chitin exhibits a high modulus and tensile strength, making it potential candidate for barrier film, bone repair materials.[266-268] Chitin as a natural polymer extracted from biomass is biodegradable and biocompatible,[269] which is ideal for cell adhesion, differentiation and proliferation.[270] There have been previous studies utilizing chitin powder for transparent food, beverage and medical packaging and bone repair materials.[271, 272] However, no study has directly utilized shell of arthropods for transparent chitin biocomposite. In this study, raw arthropods shell (shrimp and crab) were used to fabricate transparent chitin biocomposite. 


\subsection{Experiment Section}

\subsubsection{Materials and Chemicals}

Raw shrimps and snow crab purchased from local markets. $\mathrm{NaOH}, \mathrm{HCl}$ and $\mathrm{H}_{2} \mathrm{O}_{2}$ were purchased from Sigma Aldrich. PMMA was used as filtration polymers. MBA and AIBN were purchased from Sigma Aldrich. Ethanol alcohol was used for washing.

\subsubsection{Shell Treatment}

The raw shell was treated in $5 \mathrm{wt} \%$ sodium hydroxides, $7 \mathrm{wt} \%$ hydrochloric acid for 24 hours. The protein and mineral removal procedure was followed by bleaching the samples in $4 \mathrm{wt} \%$ $\mathrm{H}_{2} \mathrm{O}_{2}$ at $\mathrm{pH}$ of 8 and $100{ }^{\circ} \mathrm{C}$. The resultant chitin shell was washed under running water afterwards. The purified shell was dehydrated in the oven at $60{ }^{\circ} \mathrm{C}$ and reserved in the desiccator.

\subsubsection{Polymer Infiltration}

$5 \mathrm{ml}$ methyl methacrylate (MMA) monomer with 0.3 wt \% the initiator 2,2 - Azobis (2methylpropionitrile) (AIBN) was pre-polymerized at $70{ }^{\circ} \mathrm{C}$ for 20 minutes. The shell was infiltrated with the pre-polymerized MMA solution under vacuum for 40 minutes. The filtration was repeated for 3 times to ensure the polymer fully penetrate the shell sample. Lastly, the infiltrated shell was completed by heating the sample at $70^{\circ} \mathrm{C}$ for 4 hours. The fully polymerized shell-derived glass was peeled off from the dish. 


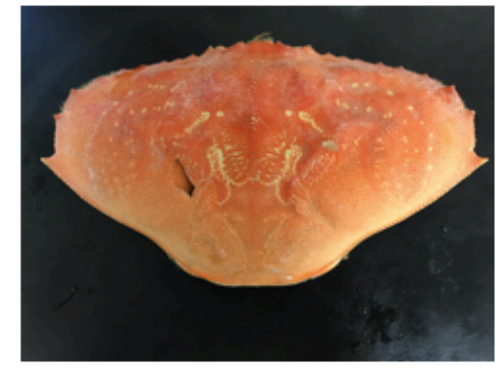

Original

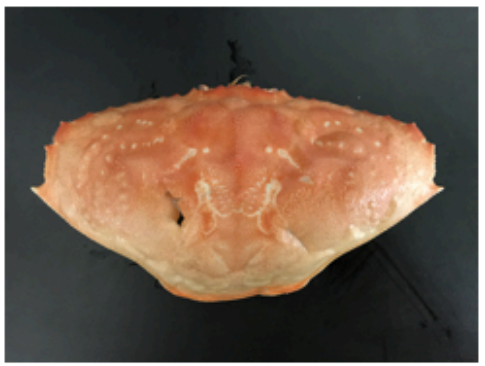

$18 \mathrm{H}$ of $12.3 \% \mathrm{HCl}$

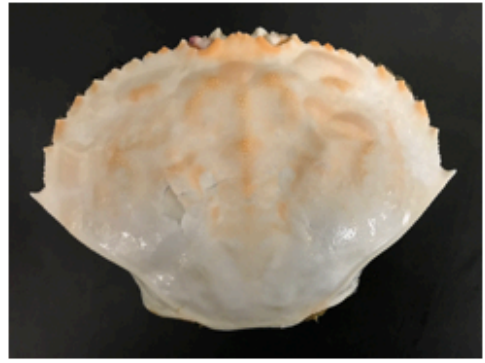

$24 \mathrm{H}$ of $8 \% \mathrm{NaOH} 3$ Times

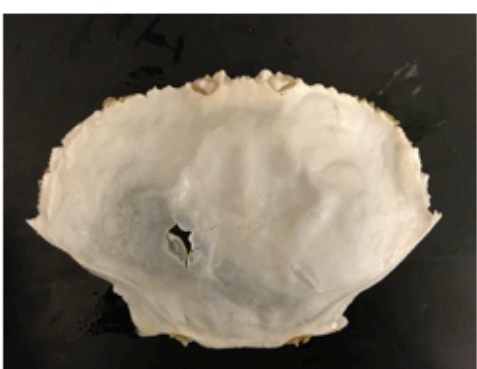

$24 \mathrm{H}$ ethanol

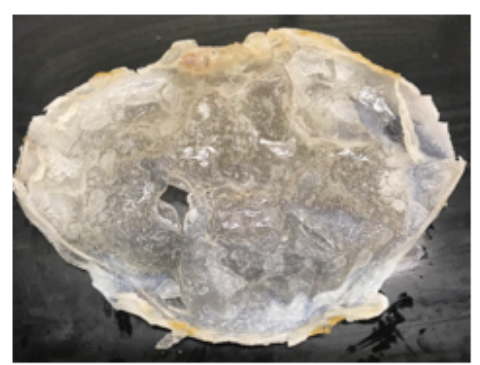

$24 \mathrm{H}$ toluene

Figure A3-1. Mineral and protein removal of the crab sample.

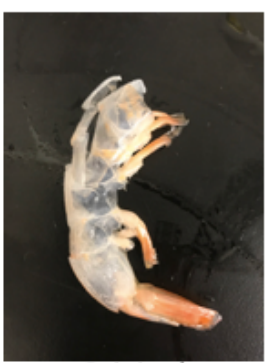

Original

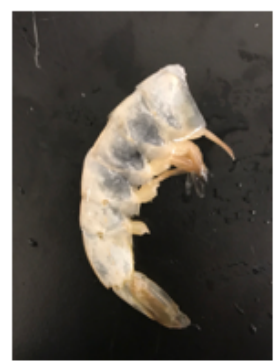

$18 \mathrm{H}$ of $12.3 \% \mathrm{HCl}$

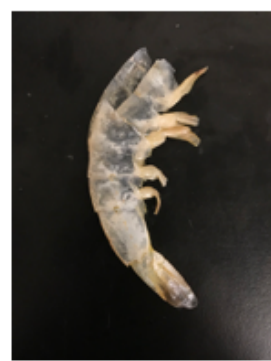

$8 \% \mathrm{NaOH} 1^{\text {st }}$ time

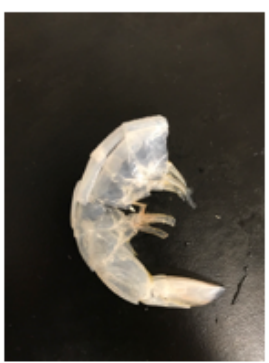

$8 \% \mathrm{NaOH} 2^{\text {nd }}$ time

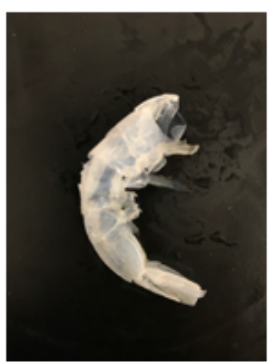

$8 \% \mathrm{NaOH} 3^{\text {rd }}$ time

Figure A3-2. Mineral and protein removal of the shrimp sample 


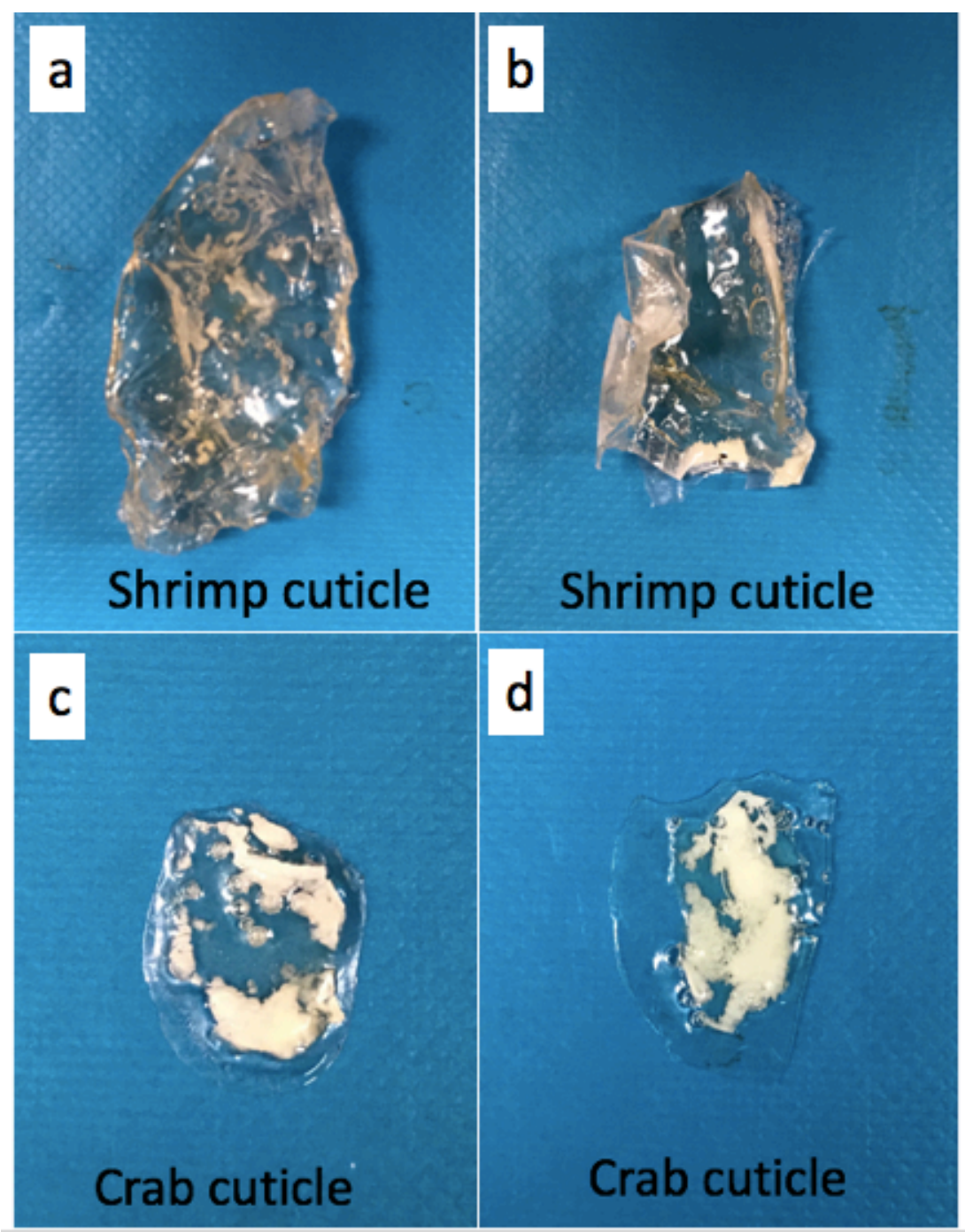

Figure A3-3. Polymerized shrimp (a,b) and crab PMMA sample (c,d).

As can be seen from Figure A3-3, both shrimp and crab samples after PMMA filtration shows moderate transparency with uneven opaque patched within the samples. The hypothesis of this result is as follows:

The crab shell constitutes only $20 \%-25 \%$ of chitin nanofibrils. The nanofibrils are only $2-5$ $\mathrm{nm}$ in diameter and approximately $20 \mathrm{~nm}$ in length. The nanofibrils are wrapped with proteins and protein-chitin fibers are embedded in protein-mineral matrix. It is highly possible that the proteins and minerals were not completely removed from the shell. This hypothesis was verified 
by increasing the shell treatment time. The samples were treated with $\mathrm{NaOH}$ and $\mathrm{HCl}$ for 48 hours, respectively. However, the improvement of transparency was not obvious.
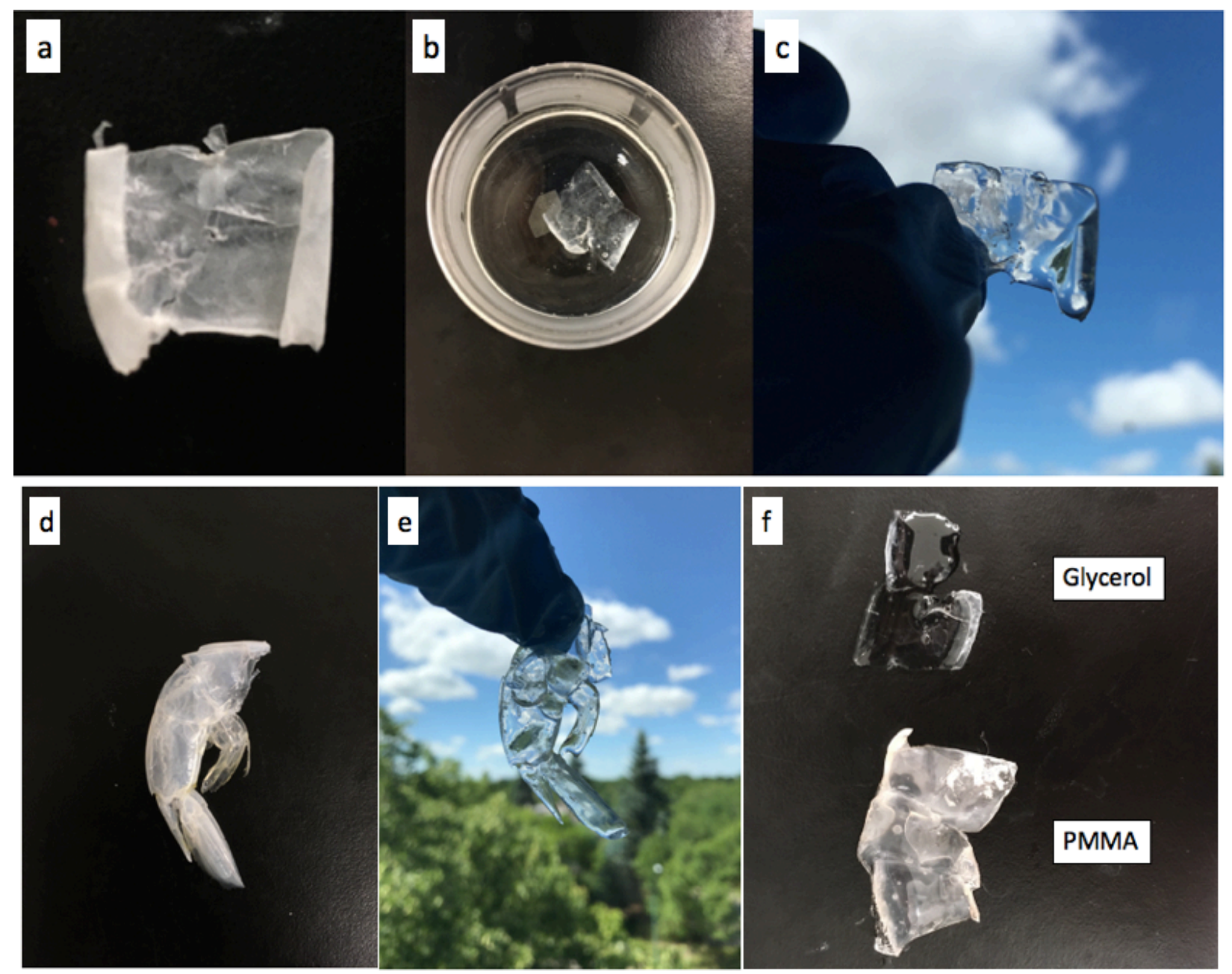

Figure A3-4. (a,d) Shrimp shell after ethanol treatment; $(\mathrm{b}, \mathrm{e})$ Shrimp shell impregnated in glycerol; (c) glycerol filtrated shrimp shell under natural light; (f) Glycerol infiltrated shrimp sample comparing with PMMA infiltrated shrimp sample.

Accidently, it was found that shrimp samples immersed in glycerol show marginally improved transparency compared to PMMA infiltrated shrimp sample. A hypothesis was made that the phenomenon was due to the refractive indexes of the crab samples and the polymer. The higher 
the refractive index, the slower the light travels, causing the materials bending the light more. The more the refractive index of the shell matches that of the polymer, the more likely the sample to achieve transparency. The refractive index of glycerol, MMA and chitin are 1.4731, 1.414 and 1.5 , respectively. The optimum refractive index of the polymer is around $1.5-1.51$ to fabricate the most transparent chitin nanocomposite.[273]

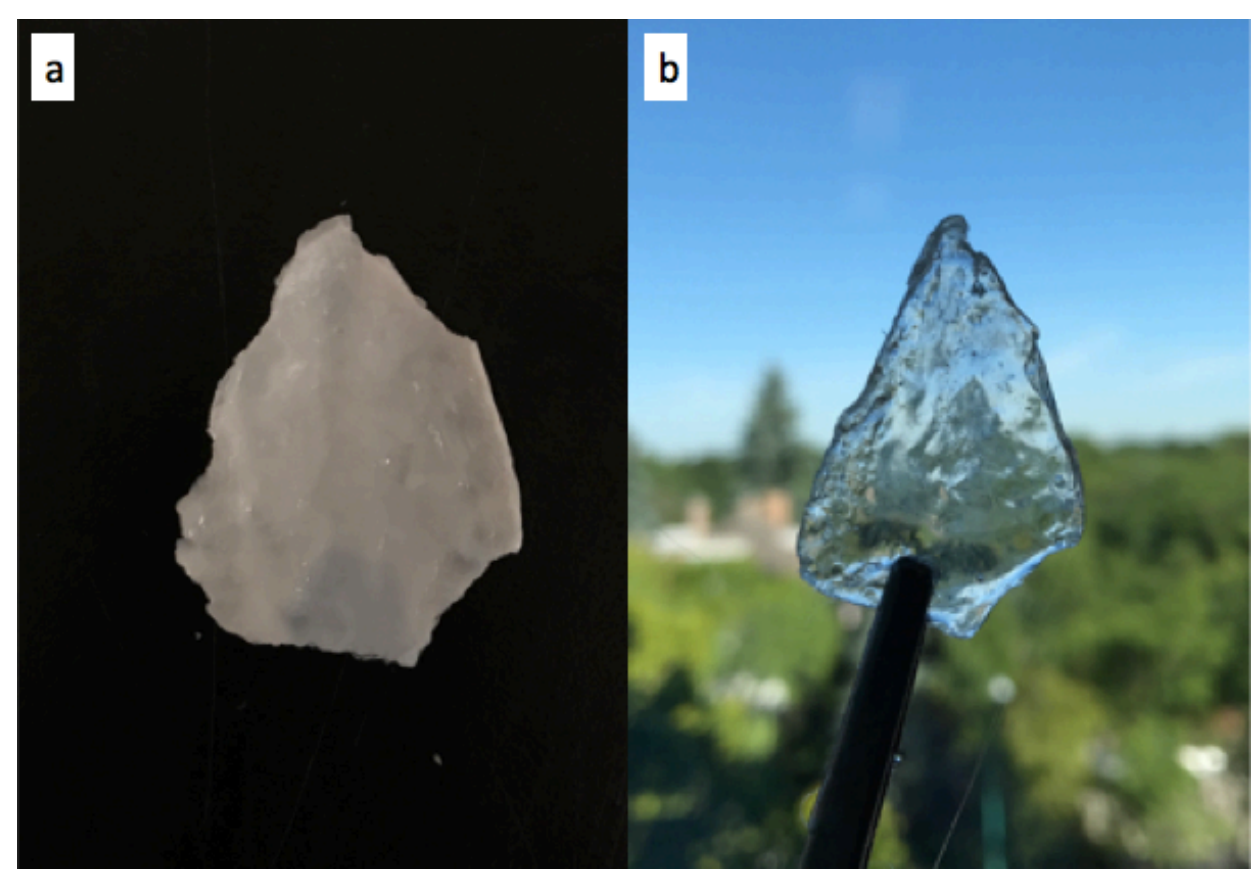

Figure A3-5. (a) Protein and mineral removed crab shell (b) Polystyrene infiltrated crab shell.

To test this hypothesis, polystyrene (PS) was infiltrated in the crab shell considering their similar refractive index. As can be seen, the PS-crab biocomposite shows marginally improved transparency comparing to PMMA infiltrated crab

\subsection{Conclusion}

Transparent arthropods-derived glass was developed for potential bio-mimetic robotic application. PMMA and PS have been utilized as infiltration materials to achieve maximum transparency. Infraction index was determined as the main factor affecting transparency of the 
resultant glass. This project can be continued with potential application in antibacterial materials, photo-sensitive electronics, etc. 